Institute Computational Mathematics

\title{
Comparison of some Entropy Conservative Numerical Fluxes for the Euler Equations
}

\author{
Hendrik Ranocha
}

28th August 2017

\begin{abstract}
Entropy conservation and stability of numerical methods in gas dynamics have received much interest. Entropy conservative numerical fluxes can be used as ingredients in two kinds of schemes: Firstly, as building blocks in the subcell flux differencing form of Fisher and Carpenter (2013) and secondly (enhanced by dissipation) as numerical surface fluxes in finite volume like schemes.

The purpose of this article is threefold. Firstly, the flux differencing theory is extended, guaranteeing high-order for general symmetric and consistent numerical fluxes and investigating entropy stability in a generalised framework of summationby-parts operators applicable to multiple dimensions and simplex elements. Secondly, a general procedure to construct affordable entropy conservative fluxes is described explicitly and used to derive several new fluxes. Finally, robustness properties of entropy stable numerical fluxes are investigated and positivity preservation is proven for several entropy conservative fluxes enhanced with local Lax-Friedrichs type dissipation operators. All these theoretical investigations are supplemented with numerical experiments.
\end{abstract}

\section{Introduction}

During the last decades, there has been an enduring and increasing interest in entropy conservation and stability of numerical methods for conservation laws. It is a topic that still needs further research and this article shall contribute to it.

As an ingredient, entropy conservative numerical fluxes can be used in two kinds of application: They can be used as volume fluxes in the flux differencing framework of Fisher and Carpenter [10] and - enhanced with additional dissipation operators - as numerical fluxes in a finite volume framework.

In this article, the theory of the flux differencing form by Fisher and Carpenter [10] is extended. Up to now, high order has only been proven for the special entropy conservative flux of Tadmor [26] but has been observed for a variety of other numerical fluxes. Here, high order of accuracy is proven in general for consistent and symmetric numerical fluxes (Theorem 3.1). Secondly, for the first time, a formulation of generalised summation-by-parts operators that can be used in multiple dimensions and on simplex elements is used to investigate entropy conservation and stability (Theorem 3.2).

Afterwards, the construction of affordable entropy conservative fluxes is briefly reviewed, a general procedure (Procedure 4.1) for their derivation is distilled and several new entropy 
conservative numerical fluxes are constructed. Nevertheless, entropy conservation or stability alone are not sufficient. In order to be robust, numerical schemes for gas dynamics also have to satisfy the physical constraints given by positivity (non-negativity) of the density and internal energy / pressure. Thus, additional dissipation / limiting will be necessary in general, possibly reducing the order of the scheme. Furthermore, general results about convergence are still unknown.

However, the aim of this article is not the investigation of convergence but the analysis of some entropy conservative and stable schemes. Therefore, the numerical fluxes are enhanced with several dissipation operators. Positivity preservation is investigated and most entropy conservative fluxes enhanced with local Lax-Friedrichs type dissipation operators are proven to preserve non-negativity of the density under a non-vanishing CFL condition (Theorem 6.1).

This article is organised as follows. At first, some well-known properties of the Euler equations are summed up in section 2 in order to fix the notation and for further reference. Afterwards, the extension of the flux differencing theory of Fisher and Carpenter [10] is presented in section 3. Thereafter, several entropy conservative numerical fluxes are constructed in sections 4 and 5 . To get numerical surface fluxes usable in finite volume methods, the addition of dissipation is discussed in section 6, especially with regard to positivity preservation. After that, the methods are tested in section 7. Finally, the results are summed up in section 8 , conclusions are drawn and some remaining open problems are formulated.

\section{Euler Equations}

In this section, some well known properties of the Euler equations in two space dimensions are given in order to fix the notation and refer to them later. The Euler equations are

$$
\partial_{t} \underbrace{\left(\begin{array}{c}
\varrho \\
\varrho v_{x} \\
\varrho v_{y} \\
\varrho e
\end{array}\right)}_{=u}+\partial_{x} \underbrace{\left(\begin{array}{c}
\varrho v_{x} \\
\varrho v_{x}^{2}+p \\
\varrho v_{x} v_{y} \\
(\varrho e+p) v_{x}
\end{array}\right)}_{=f_{x}(u)}+\partial_{y} \underbrace{\left(\begin{array}{c}
\varrho v_{y} \\
\varrho v_{x} v_{y} \\
\varrho v_{y}^{2}+p \\
(\varrho e+p) v_{y}
\end{array}\right)}_{=f_{y}(u)}=0
$$

where $\varrho$ is the density of the gas, $v=\left(v_{x}, v_{y}\right)$ its speed, $\varrho v$ the momentum, $e$ the specific total energy, and $p$ the pressure. The total energy $\varrho e$ can be decomposed into the internal energy $\varrho \varepsilon$ and the kinetic energy $\frac{1}{2} \varrho v^{2}$, i.e. $\varrho e=\varrho \varepsilon+\frac{1}{2} \varrho v^{2}$. For a perfect gas,

$$
p=\varrho R T=(\gamma-1) \varrho \varepsilon=(\gamma-1)\left(\varrho e-\frac{1}{2} \varrho v^{2}\right),
$$

where $R$ is the gas constant, $T$ the (absolute) temperature, and $\gamma$ the ratio of specific heats. For air, $\gamma=1.4$ will be used, unless stated otherwise.

The (mathematical) entropy (scaled by a constant for convenience, as chosen inter alia by $[3$, 15]) used is

$$
U=-\frac{\varrho s}{\gamma-1},
$$

where the (physical) specific entropy is given by $s=\log \frac{p}{\rho^{\gamma}}=\log p-\gamma \log \varrho$. With the associated entropy flux $F=U v=-\frac{\varrho s}{\gamma-1} v$, smooth solutions fulfil $\partial_{t} U+\partial_{x} F_{x}+\partial_{y} F_{y}=0$, and the entropy inequality

$$
\partial_{t} U+\partial_{x} F_{x}+\partial_{y} F_{y} \leq 0
$$

will be used as an additional admissibility criterion for weak solutions.

For $\varrho, p>0$, the entropy $U(u)$ is strictly convex, and the entropy variables

$$
w=U^{\prime}(u)=\left(\frac{\gamma}{\gamma-1}-\frac{s}{\gamma-1}-\frac{\varrho v^{2}}{2 p}, \frac{\varrho v_{x}}{p}, \frac{\varrho v_{y}}{p},-\frac{\varrho}{p}\right)^{T}
$$

can be used interchangeably with the conservative variables $u$. The flux potentials $\psi_{x}=\varrho v_{x}$, $\psi_{y}=\varrho v_{y}$ fulfil $\psi_{x / y}^{\prime}(w)=f_{x / y}(u(w))$ and $F_{x / y}=w \cdot f_{x / y}-\psi_{x / y}$. 


\section{Summation-by-Parts Operators and Flux Differencing}

In this section, summation-by-parts operators are briefly presented in order to fix the notation. Afterwards, the flux differencing framework of Fisher and Carpenter [10, 11] is described and new results about properties of the resulting semidiscretisations are given.

\subsection{Summation-by-Parts Operators}

Summation-by-parts (SBP) operators are composed of discrete derivative operators and quadrature rules. These differential and integral operators are compatible, i.e. they satisfy a discrete analogue of the fundamental theorem of calculus or the divergence theorem in one or several space dimensions, respectively. Since the multidimensional framework can be described nearly as briefly as in one space dimension, multiple dimensions are considered here $[14,20]$.

Numerical solutions have to be represented as a vector $\underline{u}$ in a finite dimensional vector space. In the following, nodal bases are considered, i.e. the components $\underline{u}_{i}=u\left(\xi_{i}\right)$ are nodal values at pairwise different points $\xi_{i}$. Furthermore, nonlinear operations are performed pointwise on these nodes as in classical finite difference methods. As an example, the square of the numerical solution represented as $\underline{u}$ is given as $\underline{u^{2}}$, where $\left(\underline{u}^{2}\right)_{i}=\left(\underline{u}_{i}\right)^{2}$, and the flux $\underline{f}$ is given by the components $\underline{f}_{i}=f\left(\underline{u}_{i}\right)$.

Definition 3.1. An SBP operator on a $d$ dimensional element $\Omega$ with order of accuracy $p \in \mathbb{N}$ consists of the following components.

- Derivative operators $\underline{D_{j}}, j \in\{1, \ldots, d\}$, approximating the partial derivative in the $j$-th coordinate direction. $\overline{\bar{T}}$ These are required to be exact for polynomials of degree $\leq p$.

- A mass matrix $\underline{\underline{M}}$, approximating the $L_{2}$ scalar product on $\Omega$ via

$$
\underline{u}^{T} \underline{\underline{M}} \underline{v}=\langle\underline{u}, \underline{v}\rangle_{M} \approx\langle u, v\rangle_{L_{2}(\Omega)}=\int_{\Omega} u v,
$$

where $u, v$ are functions on $\Omega$ and $\underline{u}, \underline{v}$ their approximations in the SBP basis (also known as projections on the grid).

- A restriction operator $\underline{\underline{R}}$ performing interpolation of functions on the volume $\Omega$ to the boundary $\partial \Omega$ of $\Omega$.

- A boundary mass matrix $\underline{\underline{B}}$ approximating the $L_{2}$ scalar product on $\partial \Omega$ via

$$
\underline{u}_{B}^{T} \underline{\underline{B}} \underline{v_{B}}=\left\langle\underline{u_{B}}, \underline{v_{B}}\right\rangle_{B} \approx\left\langle u_{B}, v_{B}\right\rangle_{L_{2}(\partial \Omega)}=\int_{\partial \Omega} u_{B} v_{B},
$$

where $u_{B}, v_{B}$ are functions on $\partial \Omega$ and $\underline{u_{B}}, \underline{v_{B}}$ their approximations in the SBP basis (also known as projections on the grid).

- Multiplication operators $N_{j}, j \in\{1, \ldots, d\}$, performing multiplication of functions on the boundary $\partial \Omega$ with the $\overline{\overline{j-\text { th }}}$ component $n_{j}$ of the outer unit normal. Thus, if $\underline{u}$ is the approximation of a function $\left.u\right|_{\Omega}$ in the SBP basis, $\underline{\underline{R}} \underline{u}$ is the approximation of $\left.u\right|_{\partial \Omega}$ on the boundary and $\underline{N_{j}} \underline{\underline{R}} \underline{u}$ is the approximation of $n_{j} u \overline{\overline{\mid}} \overline{\partial \Omega}$, where $n_{j}$ is the $j$-th component of the outer unit normal at $\partial \Omega$.

- The restriction and boundary operators approximate $\underline{u}^{T} \underline{\underline{R}}^{T} \underline{\underline{B}} \underline{\underline{N_{j}}} \underline{\underline{R}} \underline{v} \approx \int_{\partial \Omega} u v n_{j}$, where $n_{j}$ is the $j$-th component of the outer unit normal $n$, and this approximation has to be exact for polynomials of degree $\leq p$.

- Finally, the SBP property

$$
\underline{\underline{M}} \underline{\underline{D_{j}}}+\underline{\underline{D_{j}}} \underline{\underline{M}}=\underline{\underline{R}}^{T} \underline{\underline{B}} \underline{\underline{N_{j}}} \underline{\underline{R}}
$$

has to be fulfilled, mimicking the divergence theorem on a discrete level

$$
\int_{\Omega} u\left(\partial_{j} v\right)+\int_{\Omega}\left(\partial_{j} u\right) v \approx \underline{u}^{T} \underline{\underline{M}} \underline{\underline{D_{j}}} \underline{\underline{v}}+\underline{u}^{T} \underline{\underline{D_{j}^{T}}} \underline{\underline{M}} \underline{v}=\underline{u}^{T} \underline{\underline{R}}^{T} \underline{\underline{B}} \underline{\underline{N_{j}}} \underline{\underline{R}} \underline{v} \approx \int_{\partial \Omega} u v n_{j} .
$$


In one space dimension, the index of the derivative and multiplication operators $D_{1}, N_{1}$ will be dropped. Furthermore, the boundary matrix is the $2 \times 2$ identity matrix $\underline{\underline{B}}=\operatorname{diag}(1 \overline{\bar{a}})$ and multiplication with the outer normal is given by $\underline{\underline{N}}=\operatorname{diag}(-1,1)$.

Remark 3.1. In Definition 3.1, the order of accuracy is enforced in the usual sense (of Taylor expansions) by requirements of exactness for polynomials up to some specific degree $p$.

Remark 3.2. Multi-dimensional SBP operators can be constructed via tensor products of SBP operators in one dimension. However, genuinely multidimensional SBP operators on simplices that are not formed as tensor products of lower dimensional operators can be constructed as well [14]. However, coordinate directions $j$ are still used there, as in many numerical schemes known to the author.

Remark 3.3. Since coordinate directions $j$ are used, multidimensional semidiscretisations can be obtained via summing up the terms for each space dimension. Therefore, only one space dimension is considered in the following.

\subsection{Flux Differencing Form}

In the flux differencing form of Fisher and Carpenter [10, 11], (two-point) numerical fluxes and SBP operators are used to create high-order semidiscretisations of hyperbolic conservation laws.

Definition 3.2. A numerical flux $f^{\text {num }}$ is a Lipschitz continuous mapping $\left(u_{-}, u_{+}\right) \mapsto f^{\text {num }}\left(u_{-}, u_{+}\right)$ that is consistent with the flux $f$ of the conservation law (1), i.e. $f^{\text {num }}(u, u)=f(u)$.

Definition 3.3. A numerical flux $f^{\text {num }}$ is entropy conservative (in the sense of Tadmor $[25$, $26])$, if $\left(w_{i}-w_{k}\right) \cdot f^{\text {num }}\left(u_{i}, u_{k}\right)-\left(\psi_{i}-\psi_{k}\right)=0$. Here, $u_{i / k}$ are conserved variables, $w_{i / k}=w\left(u_{i / k}\right)$ the corresponding entropy variables and $\psi_{i / k}=\psi(u i / k)$ the flux potentials as in section 2 .

A numerical flux $f^{\text {num }}$ is entropy stable, (in the sense of Tadmor $[25,26]$ ), if $\left(w_{i}-w_{k}\right)$. $f^{\text {num }}\left(u_{i}, u_{k}\right)-\left(\psi_{i}-\psi_{k}\right) \leq 0$.

Definition 3.4. A numerical flux is symmetric, if $f^{\text {num }}\left(u_{i}, u_{k}\right)=f^{\text {num }}\left(u_{k}, u_{i}\right)$.

A general semidiscretisation of a conservation law $\partial_{t} u+\operatorname{div} f=0$ using SBP operators can be written on one element as

$$
\partial_{t} \underline{u}=-\underline{\mathrm{VOL}}-\underline{\mathrm{SURF}},
$$

where $\underline{\text { VOL }}$ are volume terms in the interior of the element and $\underline{\mathrm{SURF}}$ are surface terms coupling the elements.

Numerical fluxes are used in two different ways in semidiscretisations applying the flux differencing form of Fisher and Carpenter [10,11]. Firstly, numerical fluxes $f^{\text {num }}$ are applied at element boundaries in order to couple neighbouring elements as in finite volume and discontinuous Galerkin methods. Secondly, numerical fluxes are used in the interior of each element in order to form a discretisation of the divergence of the flux $f$. In order to distinguish these different applications of fluxes, the second kind of fluxes will be denoted $f^{\mathrm{vol}}$, since they form the volume terms of the semidiscretisation.

In the end, a semidiscretisation using the flux differencing form can be written as (10), where the volume and surface terms are given by

$$
\begin{aligned}
\underline{\mathrm{VOL}}_{i} & =\sum_{k} 2 \underline{\underline{D}}_{i, k} f^{\mathrm{vol}}\left(\underline{u}_{i}, \underline{u}_{k}\right), \\
\underline{\mathrm{SURF}} & =\underline{\underline{M}}^{-1} \underline{\underline{R}}^{T} \underline{\underline{B}} \underline{\underline{N}}\left(\underline{f}^{\mathrm{num}}-\underline{\underline{R}} \underline{f}\right) .
\end{aligned}
$$

Here, $f^{\text {num }}$ contains the numerical fluxes at the interfaces between elements. In (11), the sum $\sum_{k}$ contains contributions from all points of the nodal basis used to represent the numerical solutions. Heuristically, the factor 2 appears in the volume terms (11), since the volume flux $f^{\text {vol }}$ can be interpreted as a mean value, containing an additional factor $\frac{1}{2}$. It is justified essentially by Lemma 3.1 below. 
Remark 3.4. As mentioned in Remark 3.3, semidiscretisations in multiple space dimensions contain additional sums over each coordinate direction; the fluxes, derivative and multiplication operators have to be indexed by space dimension.

Remark 3.5. A first order finite volume method can be obtained in this setting as follows. The nodal basis uses only one node inside each element (e.g. the midpoint). Thus, the solution is constant in each element and the derivative matrix $\underline{D}$ is zero. Moreover, the $1 \times 1$ mass matrix is the length $\Delta x$ of the element. Thus, the volume terms (11) vanish and the surface terms (12) become $\underline{\mathrm{SURF}}=\frac{1}{\Delta x}\left(f_{R}^{\text {num }}-f_{L}^{\text {num }}\right)$, where $f_{L / R}^{\text {num }}$ is the numerical flux at the left $/$ right boundary of the element.

\subsection{Order of Accuracy}

Fisher and Carpenter [10, Theorem 3.1] considered diagonal-norm SBP operators including the boundary nodes and showed that the volume terms (11) of the semidiscretisation (10) are approximations to $\partial_{x} f$ of the same order of accuracy as the SBP derivative operators $\underline{\underline{D}}$, if the two-point flux $f^{\mathrm{vol}}$ used is the entropy conservative one proposed by Tadmor [26],

$$
f^{\mathrm{vol}}\left(u_{i}, u_{k}\right)=\int_{0}^{1} f\left(u\left(w\left(u_{i}\right)+t\left(w\left(u_{k}\right)-w\left(u_{i}\right)\right)\right)\right) \mathrm{d} t .
$$

Here, the following generalisation of Theorem 3.1 of [10] will be proven.

Theorem 3.1. If the numerical flux $f^{\mathrm{vol}}$ is smooth, consistent with the flux $f$, and symmetric, the volume terms (11) are an approximation to $\partial_{x} f$ of the same order of accuracy as the SBP derivative matrix $\underline{\underline{D}}$.

Remark 3.6. An analogous result holds in multiple space dimensions, if the contributions of the coordinate directions are summed up as mentioned in Remark 3.3.

In order to prove Theorem 3.1, the following Lemma will be used.

Lemma 3.1. If the numerical flux $f^{\mathrm{vol}}$ is smooth, consistent with the flux $f$, and symmetric, a power series expansion of the $m$-th component $f_{m}^{\mathrm{vol}}$ can be written as

$$
f_{m}^{\mathrm{vol}}\left(w_{i}, w_{k}\right)=f_{m}\left(w_{i}\right)+\frac{1}{2} f_{m}^{\prime}\left(w_{i}\right) \cdot\left(w_{k}-w_{i}\right)+\sum_{|\alpha| \geq 2} c_{\alpha}\left(w_{k}-w_{i}\right)^{\alpha},
$$

where multi-index notation is used, $c_{\alpha}$ are scalar coefficients, and $w$ denotes any variable, e.g. conservative variables, primitive variables, or entropy variables.

Here, multi-index notation [9, Appendix A.3] is used, i.e. the multi-index $\alpha=\left(\alpha_{1}, \ldots, \alpha_{n}\right) \in$ $\mathbb{N}_{0}^{n}$ has length $|\alpha|=\alpha_{1}+\cdots+\alpha_{n}$ and for $x \in \mathbb{R}^{n}, x^{\alpha}:=x_{1}^{\alpha_{1}} \cdots x_{n}^{\alpha_{n}}$. The last term in (14) is a sum over multi-indices $\alpha$ of length $|\alpha| \geq 2$.

Proof of Lemma 3.1. A general power series expansion of the mapping $w_{k} \mapsto f_{m}^{\mathrm{vol}}\left(w_{i}, w_{k}\right)$ around $w_{i}$ is

$$
f_{m}^{\mathrm{vol}}\left(w_{i}, w_{i}\right)+\left.\frac{\partial f_{m}^{\mathrm{vol}}\left(w_{i}, w_{k}\right)}{\partial w_{k}}\right|_{w_{k}=w_{i}} \cdot\left(w_{k}-w_{i}\right)+\sum_{|\alpha| \geq 2} c_{\alpha}\left(w_{k}-w_{i}\right)^{\alpha} .
$$

Since the numerical flux $f^{\mathrm{vol}}$ is consistent, i.e. $f_{m}^{\mathrm{vol}}\left(w_{i}, w_{i}\right)=f_{m}\left(w_{i}\right)$, it suffices to prove $\left.\partial_{w_{k}} f_{m}^{\mathrm{vol}}\left(w_{i}, w_{k}\right)\right|_{w_{k}=w_{i}}=\frac{1}{2} f_{m}^{\prime}\left(w_{i}\right)$.

Denoting the partial derivative with respect to the $l$-th component of $w_{k}$ as $\partial_{w_{k, l}} f_{m}^{\mathrm{vol}}\left(w_{i}, w_{k}\right)$,

$$
\begin{aligned}
& \left.\frac{\partial f_{m}^{\mathrm{vol}}\left(w_{i}, w_{k}\right)}{\partial w_{k, l}}\right|_{w_{k}=w_{i}=w}=\lim _{\delta \rightarrow 0} \frac{f_{m}^{\mathrm{vol}}\left(w, w+\delta e_{l}\right)-f_{m}^{\mathrm{vol}}(w, w)}{\delta} \\
= & \lim _{\delta \rightarrow 0} \frac{f_{m}^{\mathrm{vol}}\left(w+\delta e_{l}, w\right)-f_{m}^{\mathrm{vol}}(w, w)}{\delta}=\left.\frac{\partial f_{m}^{\mathrm{vol}}\left(w_{i}, w_{k}\right)}{\partial w_{i, l}}\right|_{w_{k}=w_{i}=w} .
\end{aligned}
$$


Due to this symmetry, the $l$-th component of directional derivative of the flux $f_{m}^{\mathrm{vol}}\left(w_{i}, w_{k}\right)$ at $w_{i}=w_{k}=w$ in direction $\frac{1}{\sqrt{2}}(1,1)^{T}$ is given by

$$
\begin{aligned}
& \left.\frac{2}{\sqrt{2}} \frac{\partial f_{m}^{\mathrm{vol}}\left(w_{i}, w_{k}\right)}{\partial w_{k, l}}\right|_{w_{k}=w_{i}=w}=\lim _{\delta \rightarrow 0} \frac{f_{m}^{\mathrm{vol}}\left(w+\frac{\delta}{\sqrt{2}} e_{l}, w+\frac{\delta}{\sqrt{2}} e_{l}\right)-f_{m}^{\mathrm{vol}}(w, w)}{\delta} \\
& =\lim _{\delta \rightarrow 0} \frac{f_{m}\left(w+\frac{\delta}{\sqrt{2}} e_{l}\right)-f_{m}(w)}{\delta}=\frac{1}{\sqrt{2}} \lim _{\delta \rightarrow 0} \frac{f_{m}\left(w+\delta e_{l}\right)-f_{m}(w)}{\delta}=\frac{1}{\sqrt{2}} \frac{\partial f_{m}(w)}{\partial w_{l}},
\end{aligned}
$$

where the consistency $f^{\mathrm{vol}}(w, w)=f(w)$ has been used. This proves the desired equality $\left.\partial_{w_{k}} f_{m}^{\mathrm{vol}}\left(w_{i}, w_{k}\right)\right|_{w_{k}=w_{i}}=\frac{1}{2} f_{m}^{\prime}\left(w_{i}\right)$.

Proof of Theorem 3.1. It suffices to consider a single component $m$ of the flux. In order to simplify the notation, this index is dropped in the following. Using Lemma 3.1, the volume terms (11) at $x_{i}$ can be rewritten as

$$
\begin{aligned}
\sum_{k} 2 \underline{\underline{D}}_{i, k} f^{\mathrm{vol}}\left(w_{i}, w_{k}\right)= & \sum_{k} 2 \underline{\underline{D}}_{i, k} f\left(w_{i}\right)+\sum_{k} \underline{\underline{D}}_{i, k} f^{\prime}\left(w_{i}\right) \cdot\left(w_{k}-w_{i}\right) \\
& +\sum_{k} \underline{\underline{D}}_{i, k} \sum_{|\alpha| \geq 2} c_{\alpha}\left(w_{k}-w_{i}\right)^{\alpha} .
\end{aligned}
$$

Since the derivative is exact for constants, i.e. $\underline{D} \underline{1}=0$, the first sum on the right hand side of (18) vanishes. By the same reason, the second sum can be rewritten as

$$
\sum_{k} \underline{\underline{D}}_{i, k} f^{\prime}\left(w_{i}\right) \cdot\left(w_{k}-w_{i}\right)=f^{\prime}\left(w_{i}\right) \cdot \sum_{k} \underline{\underline{D}}_{i, k} w_{k}
$$

and is therefore of the desired order of accuracy. Finally, the third summand in (18) is a higher order correction to the product rule. Due to the binomial theorem (in multi-index notation),

$$
\sum_{|\alpha| \geq 2} c_{\alpha}\left(w_{k}-w_{i}\right)^{\alpha}=\sum_{|\alpha| \geq 2} c_{\alpha} \sum_{\beta \leq \alpha}\left(\begin{array}{l}
\alpha \\
\beta
\end{array}\right) w_{k}^{\beta}\left(-w_{i}\right)^{\alpha-\beta}
$$

for multi-indices $\alpha, \beta \in \mathbb{N}_{0}^{n}$. Thus, the third term in (18) is

$$
\sum_{|\alpha| \geq 2} c_{\alpha} \sum_{\beta \leq \alpha}\left(\begin{array}{l}
\alpha \\
\beta
\end{array}\right) \sum_{k}\left(-w_{i}\right)^{\alpha-\beta} \underline{\underline{D}}_{i, k} w_{k}^{\beta},
$$

where $\beta \leq \alpha$ means $\forall j: \beta_{j} \leq \alpha_{j}$. By the product rule, a smooth function $w$ of $x$ satisfies

$$
\begin{aligned}
\partial_{x} w^{\beta}=\partial_{x}\left(w_{1}^{\beta_{1}} \ldots w_{n}^{\beta_{n}}\right) & =\sum_{j=1}^{n} \beta_{j} w_{1}^{\beta_{1}} \ldots w_{j-1}^{\beta_{j-1}} w_{j}^{\beta_{j}-1} w_{j+1}^{\beta_{j+1}} \ldots w_{n}^{\beta_{n}} \partial_{x} w_{j} \\
& =\sum_{j=1}^{n} \beta_{j} w^{\beta-e_{j}} \partial_{x} w_{j}
\end{aligned}
$$

where $e_{j}$ is the $j$-th unit vector, $\left(e_{j}\right)_{l}=\delta_{j l}$. Thus, the third sum in (18) is an approximation of the same order of accuracy as the derivative matrix $\underline{\underline{D}}$ to

$$
\begin{aligned}
& \sum_{|\alpha| \geq 2} c_{\alpha} \sum_{\beta \leq \alpha}\left(\begin{array}{l}
\alpha \\
\beta
\end{array}\right)\left(-w_{i}\right)^{\alpha-\beta} \sum_{j=1}^{n} \beta_{j} w_{i}^{\beta-e_{j}} \sum_{k} \underline{\underline{D}}_{i, k} w_{k, j} \\
= & \sum_{|\alpha| \geq 2} c_{\alpha} \sum_{j=1}^{n} \underbrace{\sum_{\beta \leq \alpha}\left(\begin{array}{l}
\alpha \\
\beta
\end{array}\right) \beta_{j}(-\mathbb{1})^{\alpha-\beta}} w_{i}^{\alpha-e_{j}} \sum_{k} \underline{\underline{D}}_{i, k} w_{k, j},
\end{aligned}
$$


where $\mathbb{1}$ is the vector with components 1 of the same size as $w_{i}$ and $w_{k, j}$ is the $j$-th component of the vector $w_{k}$ approximating $w$ at $x=x_{k}$. The sum depending on $\beta$ vanishes, since

$$
\begin{aligned}
& \partial_{w_{j}}(-\mathbb{1}+w)^{\alpha}=\partial_{w_{j}} \sum_{\beta \leq \alpha}\left(\begin{array}{l}
\alpha \\
\beta
\end{array}\right)(-\mathbb{1})^{\alpha-\beta} w^{\beta}=\sum_{\beta \leq \alpha}\left(\begin{array}{l}
\alpha \\
\beta
\end{array}\right)(-\mathbb{1})^{\alpha-\beta} \beta_{j} w^{\beta-e_{j}} \\
& \stackrel{w=\mathbb{1}}{\Longrightarrow} 0=\alpha_{j}(-\mathbb{1}+\mathbb{1})^{\alpha-e_{j}}=\sum_{\beta \leq \alpha}\left(\begin{array}{l}
\alpha \\
\beta
\end{array}\right)(-\mathbb{1})^{\alpha-\beta} \beta_{j} .
\end{aligned}
$$

Thus, the volume terms (11) are an approximation of the same order of accuracy as the derivative matrix $\underline{D}$ to $\partial_{x} f(w)$ at $x_{i}$.

\subsection{Entropy Conservation}

Fisher and Carpenter [10, Theorem 3.2] considered diagonal-norm SBP operators including the boundary nodes in one space dimension and showed that the semidiscretisation (10) using the volume terms (11) and the surface terms (12) is semidiscretely entropy conservative if the volume flux $f^{\mathrm{vol}}$ is consistent, symmetric and entropy conservative. They proved additional subcell entropy conservation properties that are not considered here, since its extension to multidimensional SBP operators on simplices does not seem clear. Instead, only entropy conservation across elements will be considered. Here, the following generalisation / variation of Theorem 3.2 of [10] will be proven.

Theorem 3.2. If the numerical (volume) flux $f^{\mathrm{vol}}$ is consistent with $f$, symmetric, and entropy conservative, the nodal mass matrix $\underline{\underline{M}}$ is diagonal, and the boundary operator $\underline{\underline{R}}^{T} \underline{\underline{B}} \underline{\underline{N}} \underline{\underline{R}}$ is diagonal, too, the semidiscrete scheme (10) is entropy conservative / stable across elements, if the numerical (surface) flux $f^{\text {num }}$ is entropy conservative / stable.

Proof of Theorem 3.2. In the semidiscrete scheme (10), the rate of change of the total entropy $\int_{\Omega} U$ is given as $\frac{\mathrm{d}}{\mathrm{d} t} \int_{\Omega} U=\underline{w}^{T} \underline{\underline{M}} \partial_{t} \underline{u}$. Multiplying the volume term (11) with $\underline{w}^{T} \underline{\underline{M}}$ results in

$$
\sum_{i, k} 2 w_{i} \cdot[\underline{\underline{M}} \underline{\underline{D}}]_{i, k} f_{i, k}^{\mathrm{vol}}=\sum_{i, k} w_{i} \cdot\left[\underline{\underline{M}} \underline{\underline{D}}+\underline{\underline{R}}^{T} \underline{\underline{B}} \underline{\underline{N}} \underline{\underline{R}}-\underline{\underline{D}}^{T} \underline{\underline{M}}\right]_{i, k} f_{i, k}^{\mathrm{vol}}
$$

where $f_{i, k}^{\mathrm{vol}}=f^{\mathrm{vol}}\left(u_{i}, u_{k}\right)$. Since the mass matrix $\underline{\underline{M}}$ is diagonal,

$$
\begin{aligned}
\sum_{i, k} w_{i} \cdot\left[\underline{\underline{M}} \underline{\underline{D}}-\underline{\underline{D}}^{T} \underline{\underline{M}}\right]_{i, k} f_{i, k}^{\mathrm{vol}} & =\sum_{i, k}\left(M_{i i} D_{i k}-M_{k k} D_{k i}\right) w_{i} \cdot f_{i, k}^{\mathrm{vol}} \\
& =\sum_{i, k} M_{i i} D_{i k}\left(w_{i}-w_{k}\right) \cdot f_{i, k}^{\mathrm{vol}},
\end{aligned}
$$

where the indices $i, j$ have been exchanged in the second part of the sum, using the symmetry of $f^{\mathrm{vol}}$. Then, by entropy conservation $\left(w_{i}-w_{k}\right) \cdot f_{i, k}^{\mathrm{vol}}=\psi_{i}-\psi_{k}$,

$$
\begin{aligned}
& \sum_{i, k} M_{i i} D_{i k}\left(w_{i}-w_{k}\right) \cdot f_{i, k}^{\mathrm{vol}}=\sum_{i, k} M_{i i} D_{i k}\left(\psi_{i}-\psi_{k}\right)=-\sum_{i, k} M_{i i} D_{i k} \psi_{k} \\
= & -\sum_{i, k}[\underline{\underline{M}} \underline{\underline{D}}]_{i k} \psi_{k}=-\sum_{i, k}\left[\underline{\underline{R}}^{T} \underline{\underline{B}} \underline{\underline{N}} \underline{\underline{R}}-\underline{\underline{D}}^{T} \underline{\underline{M}}\right]_{i k} \psi_{k}=-\sum_{i, k}\left[\underline{\underline{R}}^{T} \underline{\underline{B}} \underline{\underline{N}} \underline{\underline{R}}\right]_{i k} \psi_{k},
\end{aligned}
$$

since the derivative $\underline{D}$ is exact for constants, i.e. $\underline{D} \underline{1}=0$.

The boundary term with the diagonal matrix $\underline{\underline{R}}^{\underline{\underline{B}}} \underline{\underline{\underline{N}}} \underline{\underline{R}}$ can be written as

$$
\sum_{i, k} w_{i} \cdot\left[\underline{\underline{R}}^{T} \underline{\underline{B}} \underline{\underline{N}} \underline{\underline{R}}\right]_{i, k} f_{i, k}^{\mathrm{vol}}=\sum_{k}\left[\underline{\underline{R}}^{T} \underline{\underline{B}} \underline{\underline{N}} \underline{\underline{R}}\right]_{k, k} w_{k} \cdot \underbrace{f_{k, k}^{\mathrm{vol}}}_{=f_{k}},
$$

since the volume flux $f^{\mathrm{vol}}$ is consistent with the flux $f$. Therefore, the total expression becomes

$$
\begin{aligned}
\sum_{i, k} 2 w_{i} \cdot[\underline{\underline{M}} \underline{\underline{D}}]_{i, k} f_{i, k}^{\mathrm{vol}} & =\sum_{k}\left[\underline{\underline{R}}^{T} \underline{\underline{B}} \underline{\underline{N}} \underline{\underline{R}}\right]_{k, k} \underbrace{\left(w_{k} \cdot f_{k}-\psi_{k}\right)}_{=F_{k}} \\
& =\sum_{i, k}\left[\underline{\underline{R}}^{T} \underline{\underline{B}} \underline{\underline{N}} \underline{\underline{R}}\right]_{i, k} F_{k}=\underline{1}^{T} \underline{\underline{R}}^{T} \underline{\underline{B}} \underline{\underline{N}} \underline{\underline{R}} \underline{F},
\end{aligned}
$$


since the entropy flux $F$ is given by $F=w \cdot f-\psi$.

The surface term (12) multiplied with $\underline{w}^{T} \underline{\underline{M}}$ is $\underline{w}^{T} \underline{\underline{R}}^{T} \underline{\underline{B}} \underline{\underline{N}}\left(\underline{f}^{\text {num }}-\underline{\underline{R}} \underline{f}\right)$. Thus, the semidiscrete rate of change of the entropy $U$ in one element is

$$
\underline{w}^{T} \underline{\underline{M}} \partial_{t} \underline{u}=-\underline{1}^{T} \underline{\underline{R}}^{T} \underline{\underline{B}} \underline{\underline{N}} \underline{\underline{R}} \underline{F}-\underline{w}^{T} \underline{\underline{R}}^{T} \underline{\underline{B}} \underline{\underline{N}}\left(\underline{f}^{\text {num }}-\underline{\underline{R}} \underline{f}\right) .
$$

Since $\underline{\underline{R}}^{T} \underline{\underline{B}} \underline{\underline{N}} \underline{\underline{R}}$ is diagonal, $\underline{1}^{T} \underline{\underline{R}}^{T} \underline{\underline{B}} \underline{\underline{N}} \underline{\underline{R}} \underline{F}=\underline{w}^{T} \underline{\underline{R}}^{T} \underline{\underline{B}} \underline{\underline{N}} \underline{\underline{R}} \underline{f}-\underline{1}^{T} \underline{\underline{R}}^{T} \underline{\underline{B}} \underline{\underline{N}} \underline{\underline{R}} \underline{\psi}$. Therefore,

$$
\underline{w}^{T} \underline{\underline{M}} \partial_{t} \underline{u}=\underline{1}^{T} \underline{\underline{R}}^{T} \underline{\underline{B}} \underline{\underline{N}} \underline{\underline{R}} \underline{\psi}-\underline{w}^{T} \underline{\underline{R}}^{T} \underline{\underline{B}} \underline{\underline{N}} \underline{f}^{\text {num }} .
$$

Since the numerical flux is defined per boundary, the contribution of one boundary between cells with indices,-+ is given as

$$
\left(w_{+}-w_{-}\right) \cdot f^{\text {num }}-\left(\psi_{+}-\psi_{-}\right)
$$

which vanishes for an entropy conservative flux $f^{\text {num }}$ and is non-positive for an entropy stable flux.

Remark 3.7. A multi-dimensional analogue of Theorem 3.2 can be obtained if the contributions of the coordinate directions are summed up as mentioned in Remark 3.3. However, the assumption of diagonal mass and boundary matrices is still crucial. To the author's knowledge, there are no known SBP operators on simplices in general with diagonal $\underline{\underline{R}}^{T} \underline{\underline{B}} \underline{N_{j}} \underline{\underline{R}}$. In the framework of Hicken et al. [14], this operator is called $\mathrm{E}_{j}$ and they mention (Remark 4 in section 4.2) that they have not been able to get diagonal operators that are sufficiently accurate. However, using tensor products of Lobatto-Legendre nodes in cubes, these operators are diagonal. Additionally, it can be conjectured that it is possible to get diagonal operators $\underline{\underline{R}}^{T} \underline{\underline{B}} N_{j} \underline{\underline{R}}$ if enough nodes are added at the boundaries. However, this would probably reduce the efficiency of the scheme.

Remark 3.8. To sum up, the semidiscretisation (10) using the flux differencing form of the volume terms (11) and surface terms (12) with entropy stable numerical fluxes is entropy stable and high order accurate. However, additional dissipation will still be needed in general if discontinuities appear. Thus, it should only be considered as an entropy stable baseline scheme.

\section{Entropy Conservative Fluxes}

In the semidiscrete setting of Tadmor $[25,26]$, an entropy conservative numerical flux has to fulfil

$$
\llbracket w \rrbracket \cdot f^{\text {num }, j}-\llbracket \psi_{j} \rrbracket=0,
$$

where $w$ are the entropy variables $(5), f^{\text {num, } j}$ is the numerical flux in space direction $j, \psi_{j}$ is the flux potential in space direction $j$, and

$$
\llbracket a \rrbracket=a_{+}-a_{-}
$$

denotes the jump of a quantity, cf. Definition 3.3. Since the flux $f_{j}$ is the gradient of the potential $\psi_{j}$, i.e. $f_{j}=\partial_{w} \psi_{j}$, the condition (33) for an entropy conservative flux determines $f^{\text {num }, j}$ as an appropriate mean value of $f_{j}$. Indeed, the entropy conservative flux proposed by Tadmor [26, Equation (4.6a)] has the form of an integral mean

$$
f^{\text {num }, j}\left(w_{-}, w_{+}\right)=\int_{s=0}^{1} f_{j}\left(u\left(w_{-}+s\left(w_{+}-w_{-}\right)\right)\right) \mathrm{d} s .
$$

However, this integral mean value is difficult to compute in general. Tadmor [25, Theorem 6.1] proposed another integral mean based on a piecewise linear path in phase space to compute an integral mean similar to (35). Nevertheless, another approach will be used here.

Following the well-known proverb "Differentiation is mechanics, integration is art.", the integral mean can be exchanged by some kind of differential mean. Sadly, there is no differential 
mean value theorem giving some kind of numerical flux fulfilling (33) directly in general. However, the mean value theorem can be used for scalar variables. Indeed, if a scalar conservation law is considered, both the flux potential $\psi$ and the entropy variable $w$ in (33) are scalar. Thus, the entropy conservative flux $f^{\text {num, } j}$ is uniquely determined as $f^{\text {num, }, j}=\llbracket \psi_{j} \rrbracket / \llbracket w \rrbracket$ for $\llbracket w \rrbracket \neq 0$.

A similar procedure can be used for systems of conservation laws, where the entropy variables $w$ are vector-valued. Thus, expressing both the entropy variables and the flux potential in a common set of scalar variables (e.g. primitive variables), differential mean values can be used for each scalar variable. There are several mean values that can be used for this task. The simplest one is the arithmetic mean

$$
\{a\}=\left(a_{-}+a_{+}\right) / 2,
$$

with corresponding product and chain rule

$$
\llbracket a b \rrbracket=\left\{\{a\} \llbracket \llbracket b \rrbracket+\left\{\{b\} \llbracket \llbracket a \rrbracket, \quad \llbracket a^{2} \rrbracket=2\{\{a\} \llbracket a \rrbracket .\right.\right.
$$

This is enough to get some entropy conservative fluxes for the shallow water equations, since the entropy variables $w$ and the flux potential $\psi$ can be expressed as polynomials in both the primitive variables and the entropy variables [21]. However, this is not true for the Euler equations. Therefore, other means have to be used. Roe [22] proposed the logarithmic mean

$$
\left\{\{a\}_{\log }=\frac{a_{+}-a_{-}}{\log a_{+}-\log a_{-}},\right.
$$

described in [15], including a numerically stable implementation. The corresponding chain rule reads as

$$
\llbracket \log a \rrbracket=\llbracket a \rrbracket /\left\{\{a\}_{\log } .\right.
$$

As an example, the derivation of the entropy conservative flux of [22] is carried out in section 4.1. Thereafter, the basic idea is distilled as Procedure 4.1 in section 4.2. Afterwards, the framework of kinetic energy preserving fluxes of [16] is presented and commented in section 4.3. Finally, the entropy conservative numerical flux of [3] is given and several new fluxes are constructed. While the fluxes of [22] and [3] in sections 4.1 and 4.4 are well-known in the literature, the other ones are new.

\subsection{Using $\sqrt{\frac{\varrho}{p}}, \sqrt{\frac{\varrho}{p}} v, \sqrt{\varrho p}$ as Variables}

The entropy conservative flux of $[15,22]$ can be derived using the variables

$$
z_{1}:=\sqrt{\frac{\varrho}{p}}, \quad z_{2}:=\sqrt{\frac{\varrho}{p}} v_{x}, \quad z_{3}:=\sqrt{\frac{\varrho}{p}} v_{y}, \quad z_{5}:=\sqrt{\varrho p} .
$$

In these variables, the flux potentials $\psi_{x / y}=\varrho v_{x / y}$, the entropy $s$, and the entropy variables $w$ (5) are given by

$$
\begin{gathered}
\psi_{x}=z_{2} z_{5}, \quad \psi_{y}=z_{3} z_{5}, \quad s=-(\gamma+1) \log z_{1}-(\gamma-1) \log z_{5} \\
w=\left(\frac{\gamma}{\gamma-1}-\frac{s}{\gamma-1}-\frac{1}{2} z_{2}^{2}-\frac{1}{2} z_{3}^{2}, z_{1} z_{2}, z_{1} z_{3},-z_{1}^{2}\right)^{T} .
\end{gathered}
$$

Thus, the jumps can be expressed using the chain rules / discrete differential mean value theorems (37) and (39) as

$$
\begin{aligned}
& \llbracket w_{1} \rrbracket=-\frac{1}{\gamma-1} \llbracket s \rrbracket-\frac{1}{2} \llbracket z_{2}^{2} \rrbracket-\frac{1}{2} \llbracket z_{3}^{2} \rrbracket=\frac{\gamma+1}{\gamma-1} \llbracket \log z_{1} \rrbracket+\llbracket \log z_{5} \rrbracket-\frac{1}{2} \llbracket z_{2}^{2} \rrbracket-\frac{1}{2} \llbracket z_{3}^{2} \rrbracket \\
& =\frac{\gamma+1}{\gamma-1} \frac{1}{\left\{\left\{z_{1}\right\}_{\log }\right.} \llbracket z_{1} \rrbracket+\frac{1}{\left\{\left\{z_{5}\right\}_{\log }\right.} \llbracket z_{5} \rrbracket-\left\{\left\{z_{2}\right\} \llbracket \llbracket z_{2} \rrbracket-\left\{\left\{z_{3}\right\}\right\} \llbracket z_{3} \rrbracket,\right. \\
& \llbracket w_{2} \rrbracket=\llbracket z_{1} z_{2} \rrbracket=\left\{\left\{z_{1}\right\}\right\} \llbracket z_{2} \rrbracket+\left\{\left\{z_{2}\right\} \llbracket \llbracket z_{1} \rrbracket, \quad \llbracket w_{3} \rrbracket=\llbracket z_{1} z_{3} \rrbracket=\left\{\left\{z_{1}\right\} \llbracket \llbracket z_{3} \rrbracket+\left\{\left\{z_{3}\right\} \llbracket \llbracket z_{1} \rrbracket,\right.\right.\right.
\end{aligned}
$$




$$
\llbracket w_{4} \rrbracket=-\llbracket z_{1}^{2} \rrbracket=-2\left\{\left\{z_{1}\right\}\right\} \llbracket z_{1} \rrbracket, \quad \llbracket \psi_{x} \rrbracket=\llbracket z_{2} z_{5} \rrbracket=\left\{\left\{z_{2} \rrbracket \llbracket \llbracket z_{5} \rrbracket+\left\{\left\{z_{5}\right\}\right\} \llbracket z_{2} \rrbracket,\right.\right.
$$

and the entropy conservation conditions $\llbracket w \rrbracket \cdot f^{\text {num,x }}-\llbracket \psi_{x} \rrbracket=0(33)$ becomes ( $f^{\text {num }, y}$ analogously)

$$
\begin{aligned}
0= & \left(\frac{\gamma+1}{\gamma-1} \frac{1}{\left\{\left\{z_{1}\right\}\right\}_{\log }} f_{\varrho}^{\text {num }, x}+\left\{\left\{z_{2}\right\} f_{\varrho v_{x}}^{\text {num }, x}+\left\{\left\{z_{3}\right\} f_{\varrho v_{y}}^{\text {num }, x}-2\left\{\left\{z_{1}\right\}\right\} f_{\varrho e}^{\text {num }, x}\right) \llbracket z_{1} \rrbracket\right.\right. \\
& +\left(-\left\{\left\{z_{2}\right\}\right\} f_{\varrho}^{\text {num }, x}+\left\{\left\{z_{1}\right\}\right\} f_{\varrho v_{x}}^{\text {num }, x}-\left\{\left\{z_{5}\right\}\right\}\right) \llbracket z_{2} \rrbracket+\left(-\left\{\left\{z_{3}\right\} f_{\varrho}^{\text {num }, x}+\left\{\left\{z_{1}\right\} f_{\varrho v_{y}}^{\text {num } x}\right) \llbracket z_{3} \rrbracket\right.\right. \\
& +\left(\frac{1}{\left\{\left\{z_{5}\right\}_{\log }\right.} f_{\varrho}^{\text {num }, x}-\left\{\left\{z_{2}\right\}\right) \llbracket z_{5} \rrbracket .\right.
\end{aligned}
$$

Thus, the fluxes ( $f^{\text {num,y }}$ analogously)

$$
\begin{gathered}
f_{\varrho}^{\text {num }, x}=\left\{\left\{z_{2}\right\}\right\}\left\{\left\{z_{5}\right\}_{\log }, f_{\varrho v_{x}}^{\text {num }, x}=\frac{\left\{\left\{z_{2}\right\}\right\}}{\left\{\left\{z_{1}\right\}\right\}} f_{\varrho}^{\text {num }, x}+\frac{\left\{\left\{z_{5}\right\}\right\}}{\left\{z_{1}\right\}}, f_{\varrho v_{y}}^{\text {num }, x}=\frac{\left\{\left\{z_{3}\right\}\right\}}{\left\{\left\{z_{1}\right\}\right.} f_{\varrho}^{\text {num }, x},\right. \\
f_{\varrho e}^{\text {num }, x}=\frac{1}{2} \frac{\gamma+1}{\gamma-1} \frac{1}{\left\{\left\{z_{1}\right\}\right\}\left\{z_{1}\right\}_{\log }} f_{\varrho}^{\text {num }, x}+\frac{1}{2} \frac{\left.\left\{z_{2}\right\}\right\}}{\left\{\left\{z_{1}\right\}\right\}} f_{\varrho v_{x}}^{\text {num }, x}+\frac{1}{2} \frac{\left.\left\{z_{3}\right\}\right\}}{\left\{\left\{z_{1}\right\}\right\}} f_{\varrho v_{y}}^{\text {num }, x},
\end{gathered}
$$

proposed (in one space dimension) in $[15,22]$ can be seen to be consistent and entropy conservative. However, by this choice of variables $z$, the pressure influences the numerical density flux. As explained by Derigs et al. [6], this can lead to problems if there are discontinuities in the pressure, see also Remark 6.2 and the numerical tests in section 7 .

\subsection{General Procedure to Construct Affordable Entropy Conservative Fluxes}

The general procedure to construct affordable entropy conservative fluxes that has been mentioned in the introduction of this section has been exemplified in the previous section 4.1. Similarly, the affordable, entropy conservative numerical flux of [3] can be constructed using the same general approach that can be described as

Procedure 4.1. $\quad$ 1. Express the flux potentials $\psi$ and the entropy variables $w(5)$ using the chosen set of variables.

2. Express the jumps of $\psi, w$ as products of some mean values and jumps of the chosen variables using some kind of product/chain rule as in the mean value theorem.

\subsection{Kinetic Energy Preservation}

Besides entropy conservation / stability (cf. Definition 3.3), kinetic energy preservation has been proposed as a desirable property of numerical fluxes for the Euler equations (1) and has therefore been used as a design criterion $[3,12,16]$. The kinetic energy $\frac{1}{2} \varrho v^{2}$ satisfies (for smooth solutions)

$$
\partial_{t}\left(\frac{1}{2} \varrho v^{2}\right)+\partial_{x}\left(\frac{1}{2} \varrho v^{2} v_{x}\right)+\partial_{y}\left(\frac{1}{2} \varrho v^{2} v_{y}\right)+v_{x} \partial_{x} p+v_{y} \partial_{y} p=0 .
$$

In order to mimic this behaviour discretely in one space dimension, Jameson [16, Equation (2.23)] formulated the following condition, also used in [3, Section 3] and [12, Equation (3.23)].

Definition 4.1. A numerical flux for the Euler equations (1) is said to be kinetic energy preserving, if the momentum flux $f_{\varrho v}^{\text {num }}$ can be written as $f_{\varrho v}^{\text {num }}=\left\{\{v\} f_{\varrho}^{\text {num }}+p^{\text {num }}\right.$, where $p^{\text {num }}$ is a consistent approximation of the pressure.

Remark 4.1. Every consistent numerical flux $f_{\varrho v}^{\text {num }}$ can be written in the form required in Definition 4.1, if some differences are accepted, i.e. if $p^{\text {num }}=f_{\varrho v}^{\text {num }}-\left\{\{v\} f_{\varrho}^{\text {num }}\right.$ is accepted as numerical approximation of the pressure. Thus, Definition 4.1 alone does not seem to yield a useful criterion for the construction of numerical fluxes, i.e. the structural property "kinetic energy preserving" is not well-defined. 
Remark 4.2. In this article, some properties of numerical fluxes proposed in the literature as desirable design criteria are used, including entropy conservation / stability (Definition 3.3) and kinetic energy preservation (Definition 4.1). It is not the purpose of this article to judge these criteria or attempt to use them for convergence proofs. However, as described in Remark 4.1, the property "kinetic energy preserving" should be considered carefully. Furthermore, robustness properties of entropy conservative numerical fluxes enhanced with additional dissipation operators are investigated in section 6 . There, some fluxes fulfilling an additional structural property are proven to preserve the non-negativity of the density under a non-vanishing CFL condition.

\subsection{Using $\varrho, v, \beta$ as Variables}

Using the inverse of the temperature

$$
\beta=\frac{1}{2 R T}=\frac{\varrho}{2 p},
$$

Chandrashekar [3] derived some entropy conservative fluxes. The flux potential and the entropy variables are

$$
\begin{gathered}
\psi_{x}=\varrho v_{x}, \quad \psi_{y}=\varrho v_{y}, \\
w=\left(\frac{\gamma}{\gamma-1}-\frac{s}{\gamma-1}-\beta v^{2}, 2 \beta v_{x}, 2 \beta v_{y},-2 \beta\right)^{T}, \quad s=\log \frac{p}{\varrho^{\gamma}}=-\log \beta-(\gamma-1) \log \varrho-\log 2 .
\end{gathered}
$$

\subsubsection{Variant 1}

Writing the jumps using the chain rules (37) and (39) as

$$
\begin{aligned}
& \llbracket w_{1} \rrbracket=-\frac{1}{\gamma-1} \llbracket s \rrbracket-\llbracket \beta v^{2} \rrbracket=\llbracket \log \varrho \rrbracket+\frac{1}{\gamma-1} \llbracket \log \beta \rrbracket-\llbracket \beta v^{2} \rrbracket \\
& =\frac{1}{\left\{\{\varrho\}_{\log }\right.} \llbracket \varrho \rrbracket+\frac{1}{\gamma-1} \frac{1}{\left\{\{\beta\}_{\log }\right.} \llbracket \beta \rrbracket-\left\{\left\{v_{x}^{2}\right\}\right\} \llbracket \beta \rrbracket-\left\{\left\{v_{y}^{2}\right\}\right\} \llbracket \beta \rrbracket-2\left\{\{\beta\}\left\{\left\{v_{x}\right\}\right] \llbracket v_{x} \rrbracket-2\{\{\beta\}\}\left\{v_{y}\right\}\right\} \llbracket v_{y} \rrbracket, \\
& \llbracket w_{2} \rrbracket=2 \llbracket \beta v_{x} \rrbracket=2\left\{\{\beta\} \llbracket v_{x} \rrbracket+2\left\{\left\{v_{x}\right\} \llbracket \llbracket \beta \rrbracket,\right.\right. \\
& \llbracket w_{3} \rrbracket=2 \llbracket \beta v_{y} \rrbracket=2\left\{\{\beta\} \llbracket v_{y} \rrbracket+2\left\{\left\{v_{y}\right\}\right\} \llbracket \beta \rrbracket\right. \text {, } \\
& \llbracket w_{4} \rrbracket=-2 \llbracket \beta \rrbracket, \\
& \llbracket \psi_{x} \rrbracket=\llbracket \varrho v_{x} \rrbracket=\{\{\varrho\}\} \llbracket v_{x} \rrbracket+\left\{\left\{v_{x}\right\}\right\} \llbracket \varrho \rrbracket, \\
& \llbracket \psi_{y} \rrbracket=\llbracket \varrho v_{y} \rrbracket=\{\{\varrho\}\} \llbracket v_{y} \rrbracket+\left\{\left\{v_{y}\right\} \llbracket \llbracket \varrho \rrbracket,\right.
\end{aligned}
$$

the entropy conservation conditions $\llbracket w \rrbracket \cdot f^{\text {num, } x / y}-\llbracket \psi_{x / y} \rrbracket=0$ (33) become

$$
\begin{aligned}
& 0=\left(\frac{1}{\{\varrho\}_{\log }} f_{\varrho}^{\text {num }, x}-\left\{\left\{v_{x}\right\}\right\}\right) \llbracket \varrho \rrbracket+\left(-2\left\{\{\beta\}\left\{\left\{v_{x}\right\}\right\} f_{\varrho}^{\text {num }, x}+2\left\{\{\beta\} f_{\varrho v_{x}}^{\text {num }, x}-\{\{\varrho\}) \llbracket v_{x} \rrbracket\right.\right.\right. \\
& +\left(-2\left\{\{\beta\}\left\{\left\{v_{y}\right\}\right\} f_{\varrho}^{\text {num }, x}+2\left\{\{\beta\} f_{\varrho v_{y}}^{\text {num }, x}\right) \llbracket v_{y} \rrbracket+\left(\frac{1}{\gamma-1} \frac{1}{\{\{\beta\}} f_{\varrho}^{\text {num }, x}-\left\{\left\{v_{x}^{2}\right\}\right\} f_{\varrho}^{\text {num }, x}\right.\right.\right. \\
& \left.-\left\{\left\{v_{y}^{2}\right\}\right\} f_{\varrho}^{\text {num }, x}+2\left\{\left\{v_{x}\right\}\right\} f_{\varrho v_{x}}^{\text {num }, x}+2\left\{\left\{v_{y}\right\}\right\} f_{\varrho v_{y}}^{\text {num }, x}-2 f_{\varrho e}^{\text {num }, x}\right) \llbracket \beta \rrbracket, \\
& 0=\left(\frac{1}{\left\{\{\varrho\}_{\log }\right.} f_{\varrho}^{\text {num }, y}-\left\{\left\{v_{y}\right\}\right) \llbracket \varrho \rrbracket+\left(-2\left\{\{\beta\}\left\{\left\{v_{x}\right\}\right\} f_{\varrho}^{\text {num, } y}+2\left\{\{\beta\} f_{\varrho v_{x}}^{\text {num }, y}\right) \llbracket v_{x} \rrbracket\right.\right.\right. \\
& +\left(-2\left\{\{\beta\}\left\{\left\{v_{y}\right\}\right\} f_{\varrho}^{\text {num }, y}+2\left\{\{\beta\} f_{\varrho v_{y}}^{\text {num }, y}-\{\{\varrho\}\}\right) \llbracket v_{y} \rrbracket+\left(\frac{1}{\gamma-1} \frac{1}{\left\{\{\beta\}_{\log }\right.} f_{\varrho}^{\text {num }, y}-\left\{\left\{v_{x}^{2}\right\}\right\} f_{\varrho}^{\text {num }, y}\right.\right.\right. \\
& \left.-\left\{\left\{v_{y}^{2}\right\}\right\} f_{\varrho}^{\text {num }, y}+2\left\{\left\{v_{x}\right\}\right\} f_{\varrho v_{x}}^{\text {num }, y}+2\left\{\left\{v_{y}\right\}\right\} f_{\varrho v_{y}}^{\text {num }, y}-2 f_{\varrho e}^{\text {num }, y}\right) \llbracket \beta \rrbracket .
\end{aligned}
$$


Thus, the fluxes

$$
\begin{aligned}
& f^{\text {num }, x}\left\{\begin{array}{l}
f_{\varrho}^{\text {num }, x}=\left\{\{\varrho\}_{\log }\left\{\left\{v_{x}\right\}\right\},\right. \\
f_{\varrho v_{x}}^{\text {num }, x}=\left\{\left\{v_{x}\right\} f_{\varrho}^{\text {num }, x}+\frac{\{\{\varrho\}\}}{2\{\beta\}\}},\right. \\
f_{\varrho v_{y}}^{\text {num }, x}=\left\{\left\{v_{y}\right\}\right\} f_{\varrho}^{\text {num }, x}, \\
f_{\varrho e}^{\text {num }, x}=\frac{1}{2(\gamma-1)} \frac{1}{\left\{\{\beta\}_{l o g}\right.} f_{\varrho}^{\text {num }, x}-\frac{\left\{\left\{v_{x}^{2}\right\}+\left\{\left\{v_{y}^{2}\right\}\right\}\right.}{2} f_{\varrho}^{\text {num }, x}+\left\{\left\{v_{x}\right\}\right\} f_{\varrho v_{x}}^{\text {num }, x}+\left\{\left\{v_{y}\right\}\right\} f_{\varrho v_{y}}^{\text {num }, x},
\end{array}\right. \\
& f^{\text {num }, y}\left\{\begin{array}{l}
f_{\varrho}^{\text {num }, y}=\{\{\varrho\}\}_{\log }\left\{\left\{v_{y}\right\}\right\}, \\
f_{\varrho v_{x}}^{\text {num }, y}=\left\{\left\{v_{x}\right\} f_{\varrho}^{\text {num }, y},\right. \\
f_{\varrho v_{y}}^{\text {num }, y}=\left\{\left\{v_{y}\right\}\right\} f_{\varrho}^{\text {num }, y}+\frac{\{\lfloor\varrho\}}{2\{\{\beta\}}, \\
f_{\varrho e}^{\text {num }, y}=\frac{1}{2(\gamma-1)} \frac{1}{\left\{\{\beta\}_{\log }\right.} f_{\varrho}^{\text {num }, y}-\frac{\left\{\left\{v_{x}^{2}\right\}\right\}+\left\{\left\{v_{y}^{2}\right\}\right\}}{2} f_{\varrho}^{\text {num }, y}+\left\{\left\{v_{x}\right\}\right\} f_{\varrho v_{x}}^{\text {num }, y}+\left\{\left\{v_{y}\right\}\right\} f_{\varrho v_{y}}^{\text {num }, y},
\end{array}\right.
\end{aligned}
$$

proposed by Chandrashekar [3] can be seen to be entropy conservative. Since $p=\frac{\varrho}{2 \beta}$, they are consistent. Additionally, they are kinetic energy preserving with numerical pressure flux $p^{\text {num }}=\frac{\{\{\varrho\}}{2\{\{\beta\}}$.

\subsubsection{Variant 2}

Choosing another possibility to split the jumps

$$
\begin{aligned}
\llbracket \beta v_{x / y}^{2} \rrbracket & =\left\{\left\{\beta v_{x / y}\right\}\right\} \llbracket v_{x / y} \rrbracket+\left\{\left\{v_{x / y}\right\}\right\} \llbracket \beta v_{x / y} \rrbracket \\
& =\left\{\left\{v_{x / y}\right\}\right\} \llbracket v_{x / y} \rrbracket+\{\{\beta\}\}\left\{\left\{v_{x / y}\right\}\right\} \llbracket v_{x / y} \rrbracket+\left\{\left\{v_{x / y}\right\}\right\}^{2} \llbracket \beta \rrbracket,
\end{aligned}
$$

the entropy conservation conditions (33) can be written as

$$
\begin{aligned}
& 0=\left(\frac{1}{\left\{\lfloor\varrho\}_{\log }\right.} f_{\varrho}^{\text {num }, x}-\left\{\left\{v_{x}\right\}\right\}\right) \llbracket \varrho \rrbracket \\
& +\left(-\left\{\left\{\beta v_{x}\right\}\right\} f_{\varrho}^{\text {num }, x}-\{\{\beta\}\}\left\{v_{x}\right\}\right\} f_{\varrho}^{\text {num }, x}+2\{\{\beta\}\} f_{\varrho v_{x}}^{\text {num }, x}-\{\{\varrho\}) \llbracket v_{x} \rrbracket \\
& +\left(-\left\{\left\{\beta v_{y}\right\}\right\} f_{\varrho}^{\text {num }, x}-\left\{\{\beta\}\left\{\left\{v_{y}\right\}\right\} f_{\varrho}^{\text {num }, x}+2\left\{\{\beta\} f_{\varrho v_{y}}^{\text {num }, x}\right) \llbracket v_{y} \rrbracket\right.\right. \\
& +\left(\frac{1}{\gamma-1} \frac{1}{\{\beta \beta\}_{\log }} f_{\varrho}^{\text {num }, x}-\left\{\left\{v_{x}^{2}\right\}\right\} f_{\varrho}^{\text {num }, x}-\left\{\left\{v_{y}^{2}\right\}\right\} f_{\varrho}^{\text {num }, x}\right. \\
& +2\left\{\left\{v_{x}\right\} f_{\varrho v_{x}}^{\text {num }, x}+2\left\{\left\{v_{y}\right\}\right\} f_{\varrho v_{y}}^{\text {num }, x}-2 f_{\varrho e}^{\text {num }, x}\right) \llbracket \beta \rrbracket, \\
& 0=\left(\frac{1}{\left\{\{\varrho\}_{\log }\right.} f_{\varrho}^{\text {num }, y}-\left\{\left\{v_{y}\right\}\right\}\right) \llbracket \varrho \rrbracket \\
& +\left(-\left\{\left\{\beta v_{x}\right\}\right\} f_{\varrho}^{\text {num }, y}-\left\{\{ \beta \} \left\{\left\{v_{x}\right\} f_{\varrho}^{\text {num, }, y}+2\left\{\{\beta\} f_{\varrho v_{x}}^{\text {num }, y}\right) \llbracket v_{x} \rrbracket\right.\right.\right. \\
& +\left(-\left\{\left\{\beta v_{y}\right\}\right\} f_{\varrho}^{\text {num }, y}-\left\{\{\beta\}\left\{\left\{v_{y}\right\}\right\} f_{\varrho}^{\text {num, }, y}+2\left\{\{\beta\} f_{\varrho v_{y}}^{\text {num }, y}-\{\{\varrho\}\}\right) \llbracket v_{y} \rrbracket\right.\right. \\
& +\left(\frac{1}{\gamma-1} \frac{1}{\{\beta\}_{\log }} f_{\varrho}^{\text {num }, y}-\left\{\left\{v_{x}^{2}\right\}\right\} f_{\varrho}^{\text {num }, y}-\left\{\left\{v_{y}^{2}\right\}\right\} f_{\varrho}^{\text {num }, y}\right. \\
& +2\left\{\left\{v_{x}\right\} f_{\varrho v_{x}}^{\text {num }, y}+2\left\{\left\{v_{y}\right\} f_{\varrho v_{y}}^{\text {num }, y}-2 f_{\varrho e}^{\text {num }, y}\right) \llbracket \beta \rrbracket .\right.
\end{aligned}
$$


Thus, the fluxes

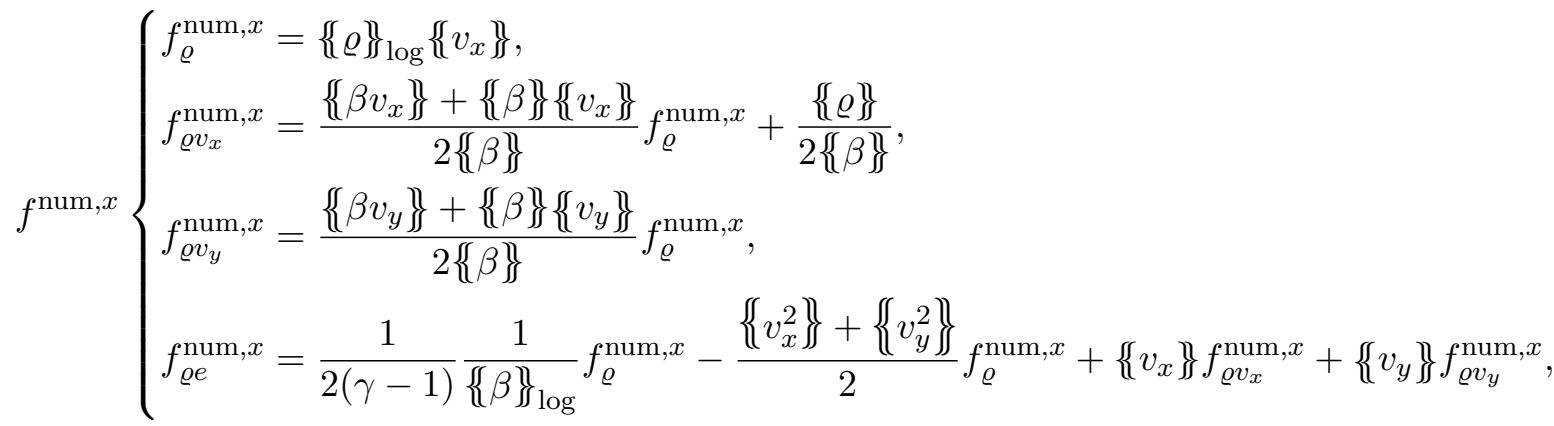

$$
\begin{aligned}
& f^{\text {num }, y}\left\{\begin{array}{l}
f_{\varrho}^{\text {num }, y}=\{\{\varrho\}\}_{\log }\left\{\left\{v_{y}\right\}\right\}, \\
f_{\varrho v_{x}}^{\text {num }, y}=\frac{\left\{\beta v_{x}\right\}+\{\{\beta\}\}\left\{\left\{v_{x}\right\}\right\}}{2\{\{\beta\}} f_{\varrho}^{\text {num }, y}, \\
f_{\varrho v_{y}}^{\text {num }, y}=\frac{\left.\left\{\beta v_{y}\right\}\right\}+\{\{\beta\}\}\left\{\left\{v_{y}\right\}\right\}}{2\{\{\beta\}} f_{\varrho}^{\text {num }, y}+\frac{\{\{\varrho\}\}}{2\{\{\beta\}}, \\
f_{\varrho e}^{\text {num }, y}=\frac{1}{2(\gamma-1)} \frac{1}{\{\{\beta\}} f_{\varrho}^{\text {num }, y}-\frac{\left.\left\{\left\{v_{x}^{2}\right\}\right\}+\left\{v_{y}^{2}\right\}\right\}}{2} f_{\varrho}^{\text {num }, y}+\left\{\left\{v_{x}\right\} f_{\varrho v_{x}}^{\text {num }, y}+\left\{\left\{v_{y}\right\} f_{\varrho v_{y}}^{\text {num }, y},\right.\right.
\end{array}\right.
\end{aligned}
$$

proposed by Chandrashekar [3] can be seen to be entropy conservative. Since the property "kinetic energy preserving" is not well-defined, they could possibly be considered as kinetic energy preserving, cf. Remark 4.1.

\subsection{Using $\varrho, v, \frac{1}{p}$ as Variables}

Using the variables $\varrho, v, \frac{1}{p}$, the flux potentials and the entropy variables (5) can be written as

$$
\begin{gathered}
\psi_{x}=\varrho v_{x}, \quad \psi_{y}=\varrho v_{y}, \\
w=\left(\frac{\gamma}{\gamma-1}-\frac{s}{\gamma-1}-\frac{\varrho v^{2}}{2 p}, \frac{\varrho v_{x}}{p}, \frac{\varrho v_{y}}{p},-\frac{\varrho}{p}\right)^{T}, \quad s=\log \frac{p}{\varrho^{\gamma}}=-\log \frac{1}{p}-\gamma \log \varrho .
\end{gathered}
$$

One variant to write the jumps is given by setting

$$
\begin{aligned}
& \llbracket w_{1} \rrbracket=-\frac{1}{\gamma-1} \llbracket s \rrbracket-\frac{1}{2} \llbracket \frac{\varrho v^{2}}{p} \rrbracket=\frac{1}{\gamma-1} \llbracket \log \frac{1}{p}+\gamma \log \varrho \rrbracket-\frac{1}{2} \llbracket \frac{\varrho v^{2}}{p} \rrbracket \\
& =\frac{1}{\gamma-1} \frac{1}{\left\{\left\{p^{-1}\right\}_{\log }\right.} \llbracket p^{-1} \rrbracket+\frac{\gamma}{\gamma-1} \frac{1}{\{\varrho \varrho\}_{\log }} \llbracket \varrho \rrbracket-\left\{\left\{\frac{\varrho}{p}\right\}\right\}\left\{\left\{v_{x}\right\} \llbracket \llbracket v_{x} \rrbracket\right. \\
& \left.-\left\{\left\{\frac{\varrho}{p}\right\}\right\}\left\{v_{y}\right\}\right\} \llbracket v_{y} \rrbracket-\left\{\{\varrho\} \frac{\left.\left\{v_{x}^{2}\right\}\right\}+\left\{\left\{v_{y}^{2}\right\}\right\}}{2} \llbracket p^{-1} \rrbracket-\frac{\left\{v_{x}^{2}\right\}+\left\{\left\{v_{y}^{2}\right\}\right.}{2}\left\{\left\{p^{-1}\right\} \llbracket \varrho \rrbracket,\right.\right. \\
& \llbracket w_{2} \rrbracket=\llbracket \frac{\varrho v_{x}}{p} \rrbracket=\left\{\left\{\frac{\varrho}{p}\right\}\right\} \llbracket v_{x} \rrbracket+\{\{\varrho\}\}\left\{\left\{v_{x}\right\}\right\} \llbracket p^{-1} \rrbracket+\left\{\left\{v_{x}\right\}\right\}\left\{\left\{p^{-1}\right\}\right\} \llbracket \varrho \rrbracket, \\
& \llbracket w_{3} \rrbracket=\llbracket \frac{\varrho v_{y}}{p} \rrbracket=\left\{\left\{\frac{\varrho}{p}\right\} \llbracket \llbracket v_{y} \rrbracket+\{\{\varrho\}\}\left\{\left\{v_{y}\right\}\right\} \llbracket p^{-1} \rrbracket+\left\{\left\{v_{y}\right\}\right\}\left\{\left\{p^{-1}\right\}\right\} \llbracket \varrho \rrbracket,\right. \\
& \llbracket w_{4} \rrbracket=-\llbracket \frac{\varrho}{p} \rrbracket=-\{\{\varrho\}\} \llbracket p^{-1} \rrbracket-\left\{\left\{p^{-1}\right\} \llbracket \llbracket \varrho \rrbracket,\right. \\
& \llbracket \psi_{x} \rrbracket=\llbracket \varrho v_{x} \rrbracket=\left\{\{\varrho\} \llbracket \llbracket v_{x} \rrbracket+\left\{\left\{v_{x}\right\}\right\} \llbracket \varrho \rrbracket,\right. \\
& \llbracket \psi_{y} \rrbracket=\llbracket \varrho v_{y} \rrbracket=\left\{\{\varrho\} \llbracket v_{y} \rrbracket+\left\{\left\{v_{y}\right\}\right\} \llbracket \varrho \rrbracket .\right.
\end{aligned}
$$


Therefore, the entropy conservation conditions (33) can be written as

$$
\begin{aligned}
& 0=\left(\frac{\gamma}{\gamma-1} \frac{1}{\left\{\{\varrho\}_{\log }\right.} f_{\varrho}^{\text {num }, x}-\frac{\left.\left\{v_{x}^{2}\right\}\right\}+\left\{\left\{v_{y}^{2}\right\}\right\}}{2}\left\{\left\{p^{-1}\right\} f_{\varrho}^{\text {num }, x}+\left\{\left\{p^{-1}\right\}\right\}\left\{v_{x}\right\}\right\} f_{\varrho v_{x}}^{\text {num }, x}\right. \\
& +\left\{\left\{p^{-1}\right\}\left\{\left\{v_{y}\right\}\right\} f_{\varrho v_{y}}^{\text {num }, x}-\left\{\left\{p^{-1}\right\} f_{\varrho e}^{\text {num }, x}-\left\{\left\{v_{x}\right\}\right) \llbracket \varrho \rrbracket\right.\right. \\
& \left.\left.\left.+\left(-\left\{\int \frac{\varrho}{p}\right\}\right\}\left\{\left\{v_{x}\right\}\right\} f_{\varrho}^{\text {num }, x}+\left\{\int \frac{\varrho}{p}\right\}\right\} f_{\varrho v_{x}}^{\text {num }, x}-\{\{\varrho\}\}\right) \llbracket v_{x} \rrbracket+\left(-\left\{\left\{\frac{\varrho}{p}\right\}\right\}\left\{\left\{v_{y}\right\}\right\} f_{\varrho}^{\text {num }, x}+\int\left\{\frac{\varrho}{p}\right\}\right\} f_{\varrho v_{y}}^{\text {num }, x}\right) \llbracket v_{y} \rrbracket \\
& +\left(\frac{1}{\gamma-1} \frac{1}{\left\{\left\{p^{-1}\right\}_{\log }\right.} f_{\varrho}^{\text {num }, x}-\{\{\varrho\}\} \frac{\left\{\left\{v_{x}^{2}\right\}\right\}+\left\{\left\{v_{y}^{2}\right\}\right\}}{2} f_{\varrho}^{\text {num }, x}+\left\{\{\varrho\}\left\{\left\{v_{x}\right\}\right\} f_{\varrho v_{x}}^{\text {num } x}\right.\right. \\
& +\left\{\{\varrho\}\left\{\left\{v_{y}\right\}\right\} f_{\varrho v_{y}}^{\text {num }, x}-\{\{\varrho\}\} f_{\varrho e}^{\text {num }, x}\right) \llbracket p^{-1} \rrbracket,
\end{aligned}
$$

$$
\begin{aligned}
& 0=\left(\frac{\gamma}{\gamma-1} \frac{1}{\left\{\{\varrho\}_{\log }\right.} f_{\varrho}^{\text {num }, y}-\frac{\left\{\left\{v_{x}^{2}\right\}\right\}+\left\{\left\{v_{y}^{2}\right\}\right\}}{2}\left\{\left\{p^{-1}\right\} f_{\varrho}^{\text {num }, y}+\left\{\left\{p^{-1}\right\}\left\{\left\{v_{x}\right\}\right\} f_{\varrho v_{x}}^{\text {num }, y}\right.\right.\right. \\
& +\left\{\left\{p^{-1}\right\}\left\{\left\{v_{y}\right\}\right\} f_{\varrho v_{y}}^{\text {num }, y}-\left\{\left\{p^{-1}\right\} f_{\varrho e}^{\text {num }, y}-\left\{\left\{v_{y}\right\}\right\}\right) \llbracket \varrho \rrbracket\right. \\
& +\left(-\left\{\left\{\frac{\varrho}{p}\right\}\right\}\left\{\left\{v_{x}\right\}\right\} f_{\varrho}^{\text {num }, y}+\left\{\left\{\frac{\varrho}{p}\right\}\right\} f_{\varrho v_{x}}^{\text {num }, y}\right) \llbracket v_{x} \rrbracket+\left(-\left\{\left\{\frac{\varrho}{p}\right\}\right\}\left\{\left\{v_{y}\right\}\right\} f_{\varrho}^{\text {num }, y}+\left\{\left\{\frac{\varrho}{p}\right\}\right\} f_{\varrho v_{y}}^{\text {num }, y}-\{\{\varrho\}) \llbracket v_{y} \rrbracket\right. \\
& +\left(\frac{1}{\gamma-1} \frac{1}{\left\{\left\{p^{-1}\right\}_{\log }\right.} f_{\varrho}^{\text {num }, y}-\left\{\{\varrho\} \frac{\left\{\left\{v_{x}^{2}\right\}\right\}+\left\{\left\{v_{y}^{2}\right\}\right\}}{2} f_{\varrho}^{\text {num }, y}+\{\{\varrho\}\}\left\{\left\{v_{x}\right\}\right\} f_{\varrho v_{x}}^{\text {num }, y}\right.\right. \\
& \left.+\{\{\varrho\}\}\left\{\left\{v_{y}\right\}\right\} f_{\varrho v_{y}}^{\text {num }, y}-\{\{\varrho\}\} f_{\varrho e}^{\text {num }, y}\right) \llbracket p^{-1} \rrbracket .
\end{aligned}
$$

Thus, the fluxes

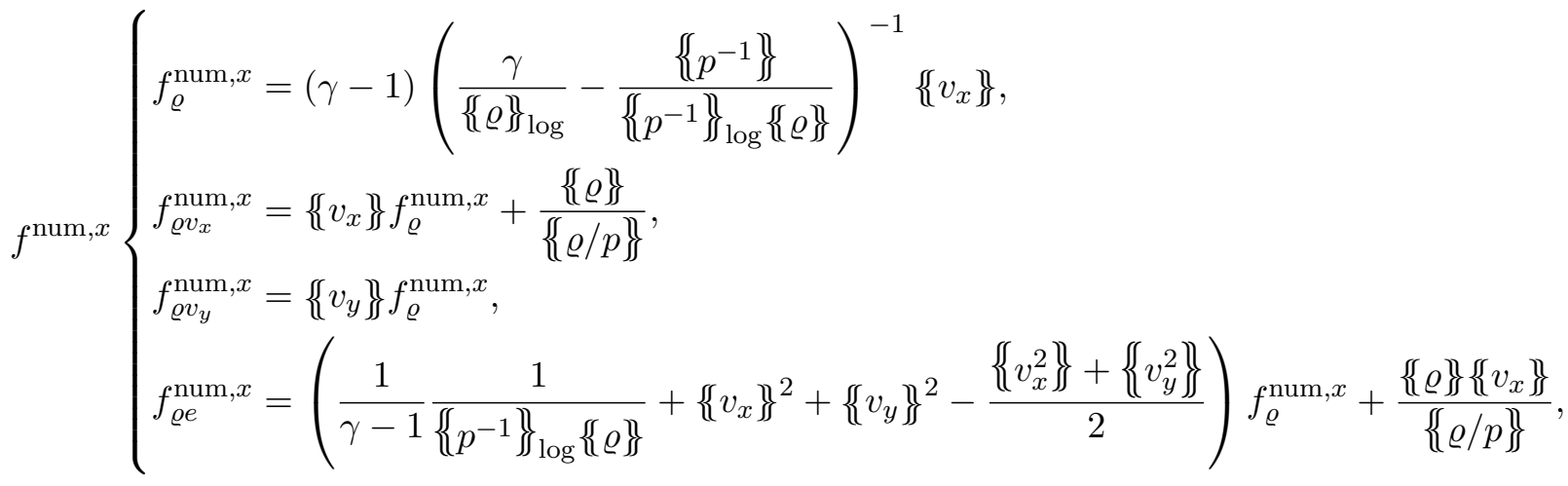

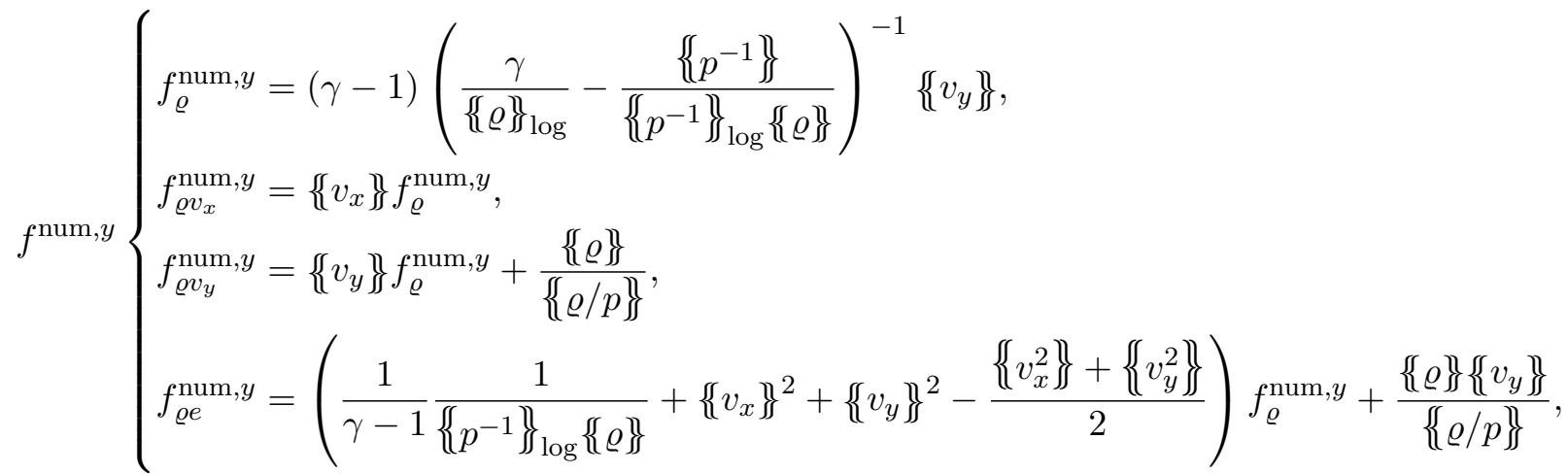


can be seen to be entropy conservative and consistent. Additionally, they are kinetic energy preserving with numerical pressure flux $p^{\text {num }}=\frac{\{\lfloor\varrho\}}{\{\{\varrho / p\}\}}$.

Again, similarly to the flux (45) of Ismail and Roe [15] and Roe [22], the pressure influences the numerical density flux, leading to some problems as explained by Derigs, Winters, Gassner, and Walch [6], see also Remark 6.2 and the numerical tests in section 7.

\subsection{Using $\varrho, v, p$ as Variables}

Using the variables $\varrho, v, p$, the flux potentials and the entropy variables (5) can be written as

$$
\begin{gathered}
\psi_{x}=\varrho v_{x}, \quad \psi_{y}=\varrho v_{y}, \\
w=\left(\frac{\gamma}{\gamma-1}-\frac{s}{\gamma-1}-\frac{\varrho v^{2}}{2 p}, \frac{\varrho v_{x}}{p}, \frac{\varrho v_{y}}{p},-\frac{\varrho}{p}\right)^{T}, \quad s=\log \frac{p}{\varrho^{\gamma}}=\log p-\gamma \log \varrho .
\end{gathered}
$$

In order to handle the terms $\frac{1}{p}$, a new mean value has to be used. Since

$$
\llbracket \frac{1}{a} \rrbracket=\frac{1}{a_{+}}-\frac{1}{a_{-}}=\frac{a_{-}-a_{+}}{a_{+} a_{-}}
$$

the geometric mean

$$
\left\{\{a\}_{\mathrm{geo}}:=\sqrt{a_{+} a_{-}},\right.
$$

fulfils

$$
\llbracket \frac{1}{a} \rrbracket=-\frac{1}{\{a\}_{\mathrm{geo}}^{2}} \llbracket a \rrbracket .
$$

One variant to write the jumps is

$$
\begin{aligned}
& \llbracket w_{1} \rrbracket=-\frac{1}{\gamma-1} \llbracket s \rrbracket-\frac{1}{2} \llbracket \frac{\varrho v^{2}}{p} \rrbracket=\frac{1}{\gamma-1} \llbracket-\log p+\gamma \log \varrho \rrbracket-\frac{1}{2} \llbracket \frac{\varrho v^{2}}{p} \rrbracket \\
& =-\frac{1}{\gamma-1} \frac{1}{\left\{\{p\}_{\log }\right.} \llbracket p \rrbracket+\frac{\gamma}{\gamma-1} \frac{1}{\left\{\{\varrho\}_{\log }\right.} \llbracket \varrho \rrbracket-\left\{\left\{\frac{\varrho}{p}\right\}\right\}\left\{\left\{v_{x}\right\} \rrbracket \llbracket v_{x} \rrbracket\right. \\
& -\left\{\left[\frac{\varrho}{p}\right\}\left\{\left\{v_{y}\right\}\right\} \llbracket v_{y} \rrbracket+\{\{\varrho]\} \frac{\left.\left\{v_{x}^{2}\right\}\right\}+\left\{\left\{v_{y}^{2}\right\}\right\}}{2\left\{\{p\}_{\text {geo }}^{2}\right.} \llbracket p \rrbracket-\frac{\left\{\left\{v_{x}^{2}\right\}\right]+\left\{\left\{v_{y}^{2}\right\}\right\}}{2}\left\{\left\{p^{-1}\right\} \llbracket \llbracket \varrho,\right.\right. \\
& \llbracket w_{2} \rrbracket=\llbracket \frac{\varrho v_{x}}{p} \rrbracket=\left\{\left\{\frac{\varrho}{p}\right\}\right\} \llbracket v_{x} \rrbracket-\frac{\{\{\varrho\}\}\left\{\left\{v_{x}\right\}\right\}}{\{p\}_{\text {geo }}^{2}} \llbracket p \rrbracket+\left\{\{ p ^ { - 1 } \} \left\{\left\{v_{x}\right\} \llbracket \llbracket \varrho \rrbracket,\right.\right. \\
& \llbracket w_{3} \rrbracket=\llbracket \frac{\varrho v_{y}}{p} \rrbracket=\left\{\left\{\frac{\varrho}{p}\right\}\right\} \llbracket v_{y} \rrbracket-\frac{\{\{\varrho\}\}\left\{\left\{v_{y}\right\}\right\}}{\left\{\{p\}_{\text {geo }}^{2}\right.} \llbracket p \rrbracket+\left\{\{ p ^ { - 1 } \} \left\{\left\{v_{y}\right\} \llbracket \llbracket \varrho \rrbracket,\right.\right. \\
& \llbracket w_{4} \rrbracket=-\llbracket \frac{\varrho}{p} \rrbracket=\frac{\{\{\varrho\}}{\{p\}_{\text {geo }}^{2}} \llbracket p \rrbracket-\left\{\left\{p^{-1}\right\} \llbracket \llbracket \varrho,\right. \\
& \llbracket \psi \rrbracket=\llbracket \varrho v \rrbracket=\{\{\varrho\} \llbracket \llbracket v \rrbracket+\{\{v\}\} \llbracket \varrho \rrbracket .
\end{aligned}
$$


Therefore, the entropy conservation conditions (33) can be written as

$$
\begin{aligned}
& 0=\left(\frac{\gamma}{\gamma-1} \frac{1}{\left\{\{\varrho\}_{\log }\right.} f_{\varrho}^{\text {num }, x}-\frac{\left\{\left\{v_{x}^{2}\right\}\right\}+\left\{\left\{v_{y}^{2}\right\}\right\}}{2}\left\{\left\{p^{-1}\right\} f_{\varrho}^{\text {num }, x}+\left\{\left\{p^{-1}\right\}\left\{\left\{v_{x}\right\}\right\} f_{\varrho v_{x}}^{\text {num }, x}\right.\right.\right. \\
& +\left\{\left\{p^{-1}\right\}\left\{\left\{v_{y}\right\}\right\} f_{\varrho v_{y}}^{\text {num }, x}-\left\{\left\{p^{-1}\right\} f_{\varrho e}^{\text {num }, x}-\left\{\left\{v_{x}\right\}\right) \llbracket \varrho \rrbracket\right.\right. \\
& \left.+\left(-\left\{\left\{\frac{\varrho}{p}\right\}\right\}\left\{\left\{v_{x}\right\}\right\} f_{\varrho}^{\text {num }, x}+\left\{\left\{\frac{\varrho}{p}\right\}\right\} f_{\varrho v_{x}}^{\text {num }, x}-\{\{\varrho\}\}\right) \llbracket v_{x} \rrbracket+\left(-\left\{\left\{\frac{\varrho}{p}\right\}\right\}\left\{\left\{v_{y}\right\}\right\} f_{\varrho}^{\text {num }, x}+\int\left\{\frac{\varrho}{p}\right\}\right\} f_{\varrho v_{y}}^{\text {num }, x}\right) \llbracket v_{y} \rrbracket \\
& +\left(-\frac{1}{\gamma-1} \frac{1}{\left\{\{p\}_{\log }\right.} f_{\varrho}^{\text {num }, x}+\{\{\varrho\}\} \frac{\left\{\left\{v_{x}^{2}\right\}\right\}+\left\{\left\{v_{y}^{2}\right\}\right\}}{2\left\{\{p\}_{\text {geo }}^{2}\right.} f_{\varrho}^{\text {num, } x}-\frac{\left\{\{\varrho\}\left\{\left\{v_{x}\right\}\right\}\right.}{\left\{\{p\}_{\text {geo }}^{2}\right.} f_{\varrho v_{x}}^{\text {num }, x}\right. \\
& \left.-\frac{\{\{\varrho\}\}\left\{\left\{v_{y}\right\}\right\}}{\left\{\{p\}_{\text {geo }}^{2}\right.} f_{\varrho v_{y}}^{\text {num }, x}+\frac{\{\{\varrho\}}{\left\{\{p\}_{\text {geo }}^{2}\right.} f_{\varrho e}^{\text {num }, x}\right) \llbracket p \rrbracket,
\end{aligned}
$$

$$
\begin{aligned}
& 0=\left(\frac{\gamma}{\gamma-1} \frac{1}{\left\{\{\varrho\}_{\log }\right.} f_{\varrho}^{\text {num }, y}-\frac{\left\{\left\{v_{x}^{2}\right\}\right\}+\left\{\left\{v_{y}^{2}\right\}\right\}}{2}\left\{\left\{p^{-1}\right\} f_{\varrho}^{\text {num }, y}+\left\{\left\{p^{-1}\right\}\left\{\left\{v_{x}\right\}\right\} f_{\varrho v_{x}}^{\text {num }, y}\right.\right.\right. \\
& +\left\{\left\{p^{-1}\right\}\left\{\left\{v_{y}\right\}\right\} f_{\varrho v_{y}}^{\text {num }, y}-\left\{\left\{p^{-1}\right\} f_{\varrho e}^{\text {num }, y}-\left\{\left\{v_{y}\right\}\right\}\right) \llbracket \varrho \rrbracket\right. \\
& +\left(-\left\{\left\{\frac{\varrho}{p}\right\}\right\}\left\{\left\{v_{x}\right\} f_{\varrho}^{\text {num }, y}+\left\{\left\{\frac{\varrho}{p}\right\}\right\} f_{\varrho v_{x}}^{\text {num }, y}\right) \llbracket v_{x} \rrbracket+\left(-\left\{\left\{\frac{\varrho}{p}\right\}\right\}\left\{v_{y}\right\}\right\} f_{\varrho}^{\text {num }, y}+\left\{\left\{\frac{\varrho}{p}\right\}\right\} f_{\varrho v_{y}}^{\text {num }, y}-\{\{\varrho\}) \llbracket v_{y} \rrbracket\right. \\
& +\left(-\frac{1}{\gamma-1} \frac{1}{\left\{\{p\}_{\log }\right.} f_{\varrho}^{\text {num }, y}+\{\{\varrho\}\} \frac{\left\{\left\{v_{x}^{2}\right\}\right\}+\left\{\left\{v_{y}^{2}\right\}\right\}}{2\left\{\{p\}_{\text {geo }}^{2}\right.} f_{\varrho}^{\text {num }, y}-\frac{\left\{\{\varrho\}\left\{\left\{v_{x}\right\}\right\}\right.}{\{p p\}_{\text {geo }}^{2}} f_{\varrho v_{x}}^{\text {num }, y}\right. \\
& \left.-\frac{\{\varrho\}\left\{\left\{v_{y}\right\}\right\}}{\left\{\{p\}_{\text {geo }}^{2}\right.} f_{\varrho v_{y}}^{\text {num }, y}+\frac{\{\{\varrho\}}{\left\{\{p\}_{\text {geo }}^{2}\right.} f_{\varrho e}^{\text {num }, y}\right) \llbracket p \rrbracket .
\end{aligned}
$$

Thus, the fluxes

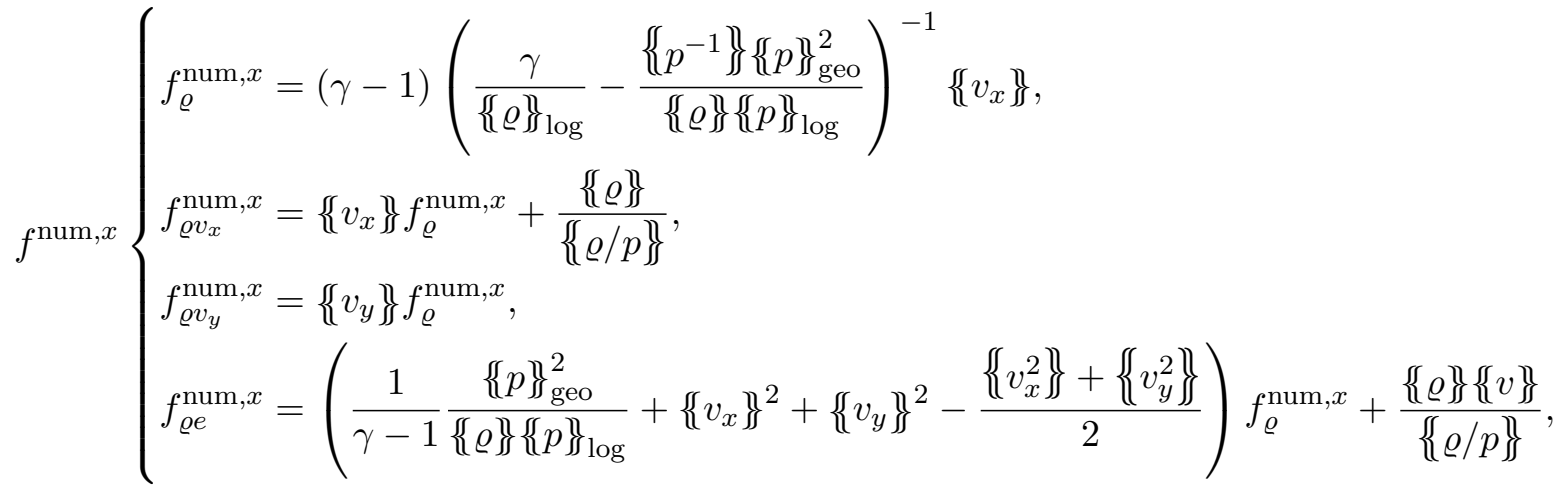

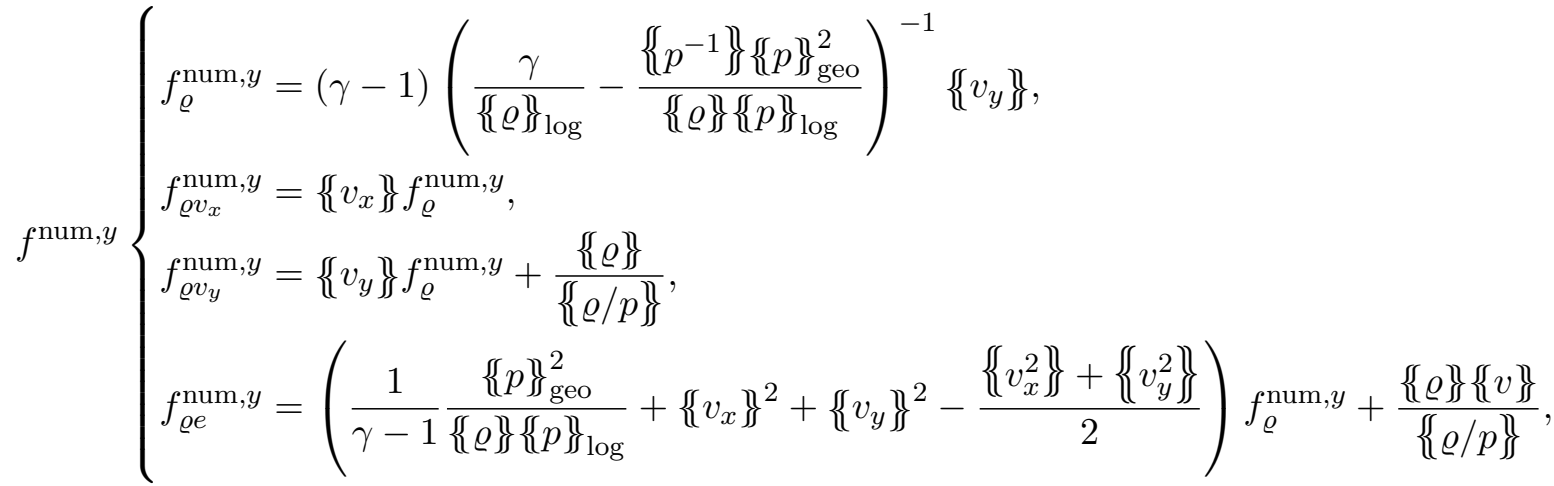


can be seen to be entropy conservative and consistent. Additionally, they are kinetic energy preserving with numerical pressure flux $p^{\text {num }}=\frac{\{\lfloor\varrho\}\}}{\{\lfloor\varrho / p\}\}}$.

As before, the pressure influences the numerical density flux, leading to some problems as explained by Derigs, Winters, Gassner, and Walch [6], see also Remark 6.2 and the numerical tests in section 7 .

\subsection{Using $\varrho, v, T$ as Variables}

Using the variables $\varrho, v$, and $R T=\frac{p}{\varrho}$, the flux potentials and the entropy variables (5) can be written as

$$
\begin{gathered}
\psi_{x}=\varrho v_{x}, \quad \psi_{y}=\varrho v_{y}, \\
w=\left(\frac{\gamma}{\gamma-1}-\frac{s}{\gamma-1}-\frac{v^{2}}{2 R T}, \frac{v_{x}}{R T}, \frac{v_{y}}{R T},-\frac{1}{R T}\right)^{T}, \quad s=\log \frac{p}{\varrho^{\gamma}}=\log R T-(\gamma-1) \log \varrho .
\end{gathered}
$$

\subsubsection{Variant 1}

One way to write the jumps is

$$
\begin{aligned}
& \llbracket w_{1} \rrbracket=-\frac{1}{\gamma-1} \llbracket s \rrbracket-\frac{1}{2} \llbracket \frac{v^{2}}{R T} \rrbracket=-\frac{1}{\gamma-1} \llbracket \log R T \rrbracket+\llbracket \log \varrho \rrbracket-\frac{1}{2} \llbracket \frac{v^{2}}{R T} \rrbracket \\
&=-\frac{1}{\gamma-1} \frac{1}{\{R T\}_{\log }} \llbracket R T \rrbracket+\frac{1}{\left\{\{\varrho\}_{\log }\right.} \llbracket \varrho \rrbracket-\left\{\frac{1}{R T}\right\}\left\{\left\{v_{x}\right\} \llbracket \llbracket v_{x} \rrbracket-\left\{\left\{\frac{1}{R T}\right\}\right\}\left\{v_{y}\right\}\right\} \llbracket v_{y} \rrbracket \\
&+\frac{\left\{\left\{v_{x}^{2}\right\}\right\}+\left\{\left\{v_{y}^{2}\right\}\right\}^{2} \llbracket R T \rrbracket,}{2\left\{\{R T\}_{\text {geo }}^{2}\right.} \\
& \llbracket w_{2} \rrbracket= \llbracket \frac{v_{x}}{R T} \rrbracket=\left\{\frac{1}{R T}\right\} \llbracket \llbracket v_{x} \rrbracket-\frac{\left\{\left\{v_{x}\right\}\right\}}{\left\{\{R T\}_{\text {geo }}^{2}\right.} \llbracket R T \rrbracket, \\
& \llbracket w_{3} \rrbracket= \llbracket \frac{v_{y}}{R T} \rrbracket=\left\{\left\{\frac{1}{R T} \rrbracket \llbracket \llbracket v_{y} \rrbracket-\frac{\left\{\left\{v_{y}\right\}\right\}}{\left\{\{R T\}_{\text {geo }}^{2}\right.} \llbracket R T \rrbracket,\right.\right. \\
& \llbracket w_{4} \rrbracket=-\llbracket \frac{1}{R T} \rrbracket=\frac{1}{\left\{\{R T\}_{\text {geo }}^{2}\right.} \llbracket R T \rrbracket, \\
& \llbracket \psi_{x} \rrbracket=\llbracket \varrho v_{x} \rrbracket=\{\{\varrho\}\} \llbracket v_{x} \rrbracket+\left\{\left\{v_{x}\right\} \llbracket \llbracket \varrho \rrbracket,\right. \\
& \llbracket \psi_{y} \rrbracket=\llbracket \varrho v_{y} \rrbracket=\left\{\{\varrho\} \llbracket \llbracket v_{y} \rrbracket+\left\{\left\{v_{y}\right\} \llbracket \llbracket \varrho \rrbracket .\right.\right.
\end{aligned}
$$


Therefore, the entropy conservation conditions (33) can be written as

$$
\begin{aligned}
& 0=\left(\frac{1}{\left\{\{\varrho\}_{\log }\right.} f_{\varrho}^{\text {num }, x}-\left\{\left\{v_{x}\right\}\right) \llbracket \varrho \rrbracket+\left(-\left\{\left\{\frac{1}{R T}\right\}\right\}\left\{\left\{v_{x}\right\} f_{\varrho}^{\text {num }, x}+\left\{\left\{\frac{1}{R T}\right\}\right\} f_{\varrho v_{x}}^{\text {num }, x}-\{\{\varrho\}) \llbracket v_{x} \rrbracket\right.\right.\right. \\
& +\left(-\left\{\left\{\frac{1}{R T}\right\}\right\}\left\{v_{y}\right\}\right\} f_{\varrho}^{\text {num }, x}+\left\{\left\{\frac{1}{R T}\right\} f_{\varrho}^{\text {num }, x}\right) \llbracket v_{y} \rrbracket \\
& +\left(-\frac{1}{\gamma-1} \frac{1}{\left\{\{R T\}_{\log }\right.} f_{\varrho}^{\text {num }, x}+\frac{\left\{\left\{v_{x}^{2}\right\}\right\}+\left\{\left\{v_{y}^{2}\right\}\right\}}{2\left\{\{R T\}_{\text {geo }}^{2}\right.} f_{\varrho}^{\text {num }, x}-\frac{\left\{\left\{v_{x}\right\}\right\}}{\left\{\{R T\}_{\text {geo }}^{2}\right.} f_{\varrho v_{x}}^{\text {num }, x}\right. \\
& \left.-\frac{\left\{\left\{v_{y}\right\}\right\}}{\left\{\{R T\}_{\text {geo }}^{2}\right.} f_{\varrho v_{y}}^{\text {num }, x}+\frac{1}{\left\{\{R T\}_{\text {geo }}^{2}\right.} f_{\varrho e}^{\text {num }, x}\right) \llbracket R T \rrbracket, \\
& 0=\left(\frac{1}{\left\{\{\varrho\}_{\log }\right.} f_{\varrho}^{\text {num }, y}-\left\{\left\{v_{y}\right\}\right) \llbracket \varrho \rrbracket+\left(-\left\{\left\{\frac{1}{R T}\right\}\right\}\left\{\left\{v_{x}\right\}\right\} f_{\varrho}^{\text {num }, y}+\left\{\left\{\frac{1}{R T}\right\}\right\} f_{\varrho v_{x}}^{\text {num }, y}\right) \llbracket v_{x} \rrbracket\right. \\
& +\left(-\left\{\frac{1}{R T}\right\}\left\{\left\{v_{y}\right\} f_{\varrho}^{\text {num }, y}+\left\{\left\{\frac{1}{R T}\right\}\right\} f_{\varrho v_{y}}^{\text {num }, y}-\{\{\varrho\}) \llbracket v_{y} \rrbracket\right.\right. \\
& +\left(-\frac{1}{\gamma-1} \frac{1}{\left\{\{R T\}_{\log }\right.} f_{\varrho}^{\text {num }, y}+\frac{\left\{\left\{v_{x}^{2}\right\}\right\}+\left\{\left\{v_{y}^{2}\right\}\right\}}{2\left\{\{R T\}_{\text {geo }}^{2}\right.} f_{\varrho}^{\text {num, }, y}-\frac{\left\{\left\{v_{x}\right\}\right\}}{\left\{\{R T\}_{\text {geo }}^{2}\right.} f_{\varrho v_{x}}^{\text {num }, y}\right. \\
& \left.-\frac{\left\{\left\{v_{y}\right\}\right.}{\left\{\{R T\}_{\text {geo }}^{2}\right.} f_{\varrho v_{y}}^{\text {num }, y}+\frac{1}{\left\{\{R T\}_{\text {geo }}^{2}\right.} f_{\varrho e}^{\text {num }, y}\right) \llbracket R T \rrbracket .
\end{aligned}
$$

Thus, the fluxes

$$
\begin{aligned}
& f^{\text {num }, x}\left\{\begin{array}{l}
f_{\varrho}^{\text {num }, x}=\{\{\varrho\}\}_{\log }\left\{\left\{v_{x}\right\}\right\}, \\
f_{\varrho v_{x}}^{\text {num }, x}=\left\{\left\{v_{x}\right\}\right\} f_{\varrho}^{\text {num }, x}+\frac{\{\lfloor\varrho\}}{\{1 / R T\}}, \\
f_{\varrho v_{y}}^{\text {num }, x}=\left\{\left\{v_{y}\right\}\right\} f_{\varrho}^{\text {num }, x}, \\
f_{\varrho e}^{\text {num }, x}=\left(\frac{1}{\gamma-1} \frac{\{R T\}_{\text {geo }}^{2}}{\{\{R T\}\}_{\text {log }}}-\frac{\left\{\left\{v_{x}^{2}\right\}\right\}+\left\{\left\{v_{y}^{2}\right\}\right\}}{2}\right) f_{\varrho}^{\text {num }, x}+\left\{\left\{v_{x}\right\}\right\} f_{\varrho v_{x}}^{\text {num }, x}+\left\{\left\{v_{y}\right\}\right\} f_{\varrho v_{y}}^{\text {num }, x},
\end{array}\right. \\
& f^{\text {num }, y}\left\{\begin{array}{l}
f_{\varrho}^{\text {num }, y}=\{\{\varrho\}\}_{\log }\left\{\left\{v_{y}\right\}\right\}, \\
f_{\varrho v_{x}, y}^{\text {num }}=\left\{\left\{v_{x}\right\}\right\} f_{\varrho}^{\text {num }, y}, \\
f_{\varrho v_{y}, y}^{\text {num }}=\left\{\left\{v_{y}\right\}\right\} f_{\varrho}^{\text {num }, y}+\frac{\{\{\varrho\}}{\{\{1 / R T\}}, \\
f_{\varrho e}^{\text {num }, y}=\left(\frac{1}{\gamma-1} \frac{\{R T\}\}_{\text {geo }}^{2}}{\{\{R T\}\}_{\text {log }}}-\frac{\left\{\left\{v_{x}^{2}\right\}\right\}+\left\{\left\{v_{y}^{2}\right\}\right\}}{2}\right) f_{\varrho}^{\text {num }, y}+\left\{\left\{v_{x}\right\}\right\} f_{\varrho v_{x}}^{\text {num }, y}+\left\{\left\{v_{y}\right\}\right\} f_{\varrho v_{y}}^{\text {num }, y},
\end{array}\right.
\end{aligned}
$$

can be seen to be entropy conservative and consistent. Additionally, they are kinetic energy preserving with numerical pressure flux $p^{\text {num }}=\frac{\{\{\varrho\}}{\{1 / R T\}}$, i.e. the same as for the entropy conservative and kinetic energy preserving flux (52) proposed by Chandrashekar [3]. Moreover, the density flux $f_{\varrho}^{\text {num }}$ is the same. However, the energy fluxes $f_{\varrho e}^{\text {num }}$ are different. 


\subsubsection{Variant 2}

Similarly to the derivation of (55), choosing another possibility to split the jump

$$
\begin{aligned}
\llbracket \frac{v_{x / y}^{2}}{R T} \rrbracket & =\left\{\left\{\frac{v_{x / y}}{R T}\right\}\right\rfloor \llbracket v_{x / y} \rrbracket+\left\{\left\{v_{x / y}\right\}\right\} \llbracket \frac{v_{x / y}}{R T} \rrbracket \\
& =\left\{\left\{\frac{v_{x / y}}{R T}\right\}\right\} \llbracket v_{x / y} \rrbracket+\left\{\left\{\frac{1}{R T}\right\}\right\}\left\{\left\{v_{x / y}\right\}\right\} \llbracket v_{x / y} \rrbracket-\frac{\left\{\left\{v_{x / y}\right\}\right\}^{2}}{\{R T\}_{\text {geo }}^{2}} \llbracket R T \rrbracket,
\end{aligned}
$$

the entropy conservation conditions (33) can be written as

$$
\begin{aligned}
& 0=\left(\frac{1}{\left\{\lfloor\varrho\}_{\log }\right.} f_{\varrho}^{\text {num }, x}-\left\{\left\{v_{x}\right\}\right\}\right) \llbracket \varrho \rrbracket \\
& +\left(-\frac{1}{2}\left\{\left\{\frac{v_{x}}{R T}\right\}\right\} f_{\varrho}^{\text {num }, x}-\frac{1}{2}\left\{\frac{1}{R T}\right\}\left\{\left\{v_{x}\right\} f_{\varrho}^{\text {num }, x}+\left\{\frac{1}{R T}\right\} f_{\varrho v_{x}}^{\text {num }, x}-\{\{\varrho\}\}\right) \llbracket v_{x} \rrbracket\right. \\
& +\left(-\frac{1}{2}\left\{\left\{\frac{v_{y}}{R T}\right\}\right\} f_{\varrho}^{\text {num }, x}-\frac{1}{2}\left\{\left\{\frac{1}{R T}\right\}\left\{\left\{v_{y}\right\}\right\} f_{\varrho}^{\text {num }, x}+\left\{\left\{\frac{1}{R T}\right\}\right\} f_{\varrho v_{y}}^{\text {num }, x}\right) \llbracket v_{y} \rrbracket\right. \\
& +\left(-\frac{1}{\gamma-1} \frac{1}{\left\{\{R T\}_{\log }\right.} f_{\varrho}^{\text {num }, x}+\frac{\left\{\left\{v_{x}\right\}\right\}^{2}+\left\{\left\{v_{y}^{2}\right\}\right\}}{2\left\{\{R T\}_{\text {geo }}^{2}\right.} f_{\varrho}^{\text {num }, x}-\frac{\left\{\left\{v_{x}\right\}\right\}}{\left\{\{R T\}_{\text {geo }}^{2}\right.} f_{\varrho v_{x}}^{\text {num }, x}\right. \\
& \left.-\frac{\left\{\left\{v_{y}\right\}\right\}}{\left\{\{R T\}_{\text {geo }}^{2}\right.} f_{\varrho v_{y}}^{\text {num }, x}+\frac{1}{\left\{\{R T\}_{\text {geo }}^{2}\right.} f_{\varrho e}^{\text {num }, x}\right) \llbracket R T \rrbracket, \\
& 0=\left(\frac{1}{\left\{\lfloor\varrho\}_{\log }\right.} f_{\varrho}^{\text {num, } y}-\left\{\left\{v_{y}\right\}\right\}\right) \llbracket \varrho \rrbracket \\
& \left.+\left(-\frac{1}{2}\left\{\left\{\frac{v_{x}}{R T}\right\}\right\} f_{\varrho}^{\text {num }, y}-\frac{1}{2}\left\{\left\{\frac{1}{R T}\right\}\right\}\left\{\left\{v_{x}\right\}\right\} f_{\varrho}^{\text {num }, y}+\left\{\frac{1}{R T}\right\}\right\} f_{\varrho v_{x}}^{\text {num }, y}\right) \llbracket v_{x} \rrbracket \\
& +\left(-\frac{1}{2}\left\{\left\{\frac{v_{y}}{R T}\right\}\right\} f_{\varrho}^{\text {num }, y}-\frac{1}{2}\left\{\left\{\frac{1}{R T}\right\}\right\}\left\{v_{y}\right\}\right\} f_{\varrho}^{\text {num }, y}+\left\{\left\{\frac{1}{R T}\right\}\right\} f_{\varrho v_{y}}^{\text {num }, y}-\{\{\varrho\}) \llbracket v_{y} \rrbracket \\
& +\left(-\frac{1}{\gamma-1} \frac{1}{\left\{\{R T\}_{\log }\right.} f_{\varrho}^{\text {num }, y}+\frac{\left\{\left\{v_{x}\right\}\right\}^{2}+\left\{\left\{v_{y}^{2}\right\}\right\}}{2\left\{\{R T\}_{\text {geo }}^{2}\right.} f_{\varrho}^{\text {num }, y}-\frac{\left\{\left\{v_{x}\right\}\right\}}{\left\{\{R T\}_{\text {geo }}^{2}\right.} f_{\varrho v_{x}}^{\text {num }, y}\right. \\
& \left.-\frac{\left\{\left\{v_{y}\right\}\right\}}{\left\{\{R T\}_{\text {geo }}^{2}\right.} f_{\varrho v_{y}}^{\text {num }, y}+\frac{1}{\left\{\{R T\}_{\text {geo }}^{2}\right.} f_{\varrho e}^{\text {num }, y}\right) \llbracket R T \rrbracket .
\end{aligned}
$$


Thus, the fluxes

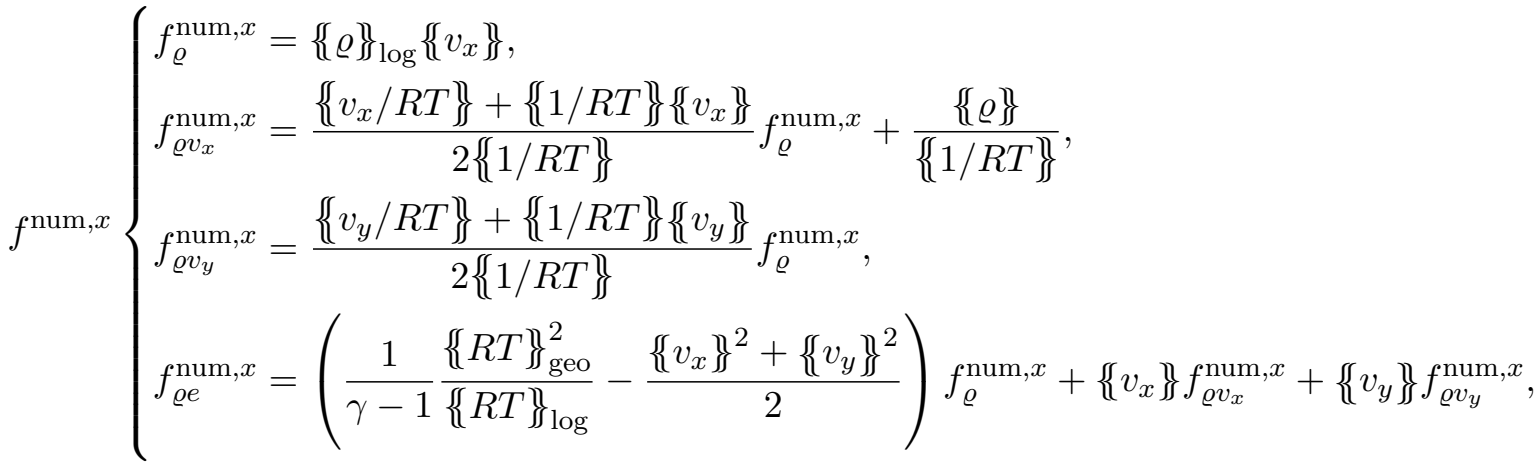

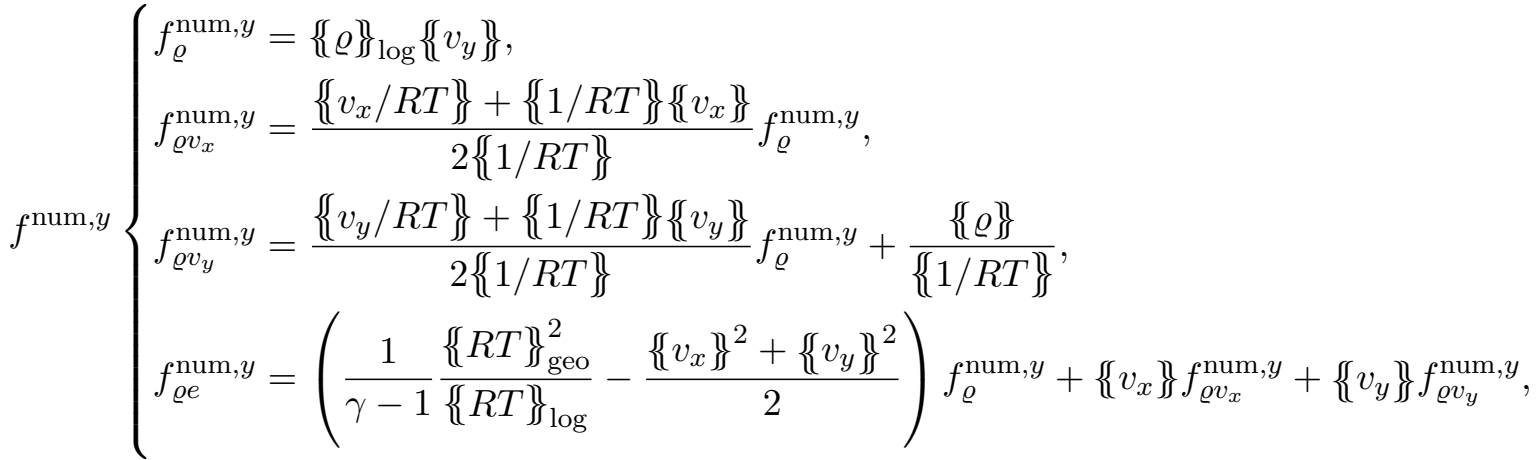

can be seen to be entropy conservative. Again, since the property "kinetic energy preserving" is not well-defined, they could possibly be considered as kinetic energy preserving, cf. Remark 4.1.

\subsection{Using $\varrho, v, g^{-1}(\varrho / p)$ as Variables}

As can be seen in the previous subsections, there are many entropy conservative and kinetic energy preserving numerical fluxes in the sense described at the beginning of section 4 , obtained using the general procedure described there. However, they are different and will thus have advantages or disadvantages compared to each other. Looking at the entropy variables (5)

$$
w=\left(\frac{\gamma}{\gamma-1}-\frac{s}{\gamma-1}-\frac{\varrho v^{2}}{2 p}, \frac{\varrho v_{x}}{p}, \frac{\varrho v_{y}}{p},-\frac{\varrho}{p}\right)^{T}, \quad s=\log \frac{p}{\varrho^{\gamma}},
$$

it can be seen that the term $\frac{\varrho}{p}$ has a crucial role. If the variables $\varrho, v, \chi$ are chosen (where $\chi$ is some third variable), and the expression $\frac{\varrho}{p}=g(\chi)$ depends only on this third variable $\chi$, a kinetic energy preserving flux can be constructed using a density flux depending only on $\varrho, v$. Indeed, writing the jumps as

$$
\begin{aligned}
& \llbracket w_{1} \rrbracket=-\frac{1}{\gamma-1} \llbracket s \rrbracket-\frac{1}{2} \llbracket \frac{\varrho}{p} v^{2} \rrbracket=\llbracket \log \varrho \rrbracket+\frac{1}{\gamma-1} \llbracket \log \frac{\varrho}{p} \rrbracket-\frac{1}{2} \llbracket \frac{\varrho}{p} v^{2} \rrbracket \\
& =\frac{1}{\left\{\{\varrho\}_{\log }\right.} \llbracket \varrho \rrbracket+\frac{1}{\gamma-1} \llbracket \log \frac{\varrho}{p} \rrbracket-\frac{\left\{\left\{v_{x}^{2}\right\}+\left\{\left\{v_{y}^{2}\right\}\right\}\right.}{2} \llbracket \frac{\varrho}{p} \rrbracket-\left\{\left\{\frac{\varrho}{p}\right\}\right\}\left\{\left\{v_{x}\right\}\right\} \llbracket v_{x} \rrbracket-\left\{\left\{\frac{\varrho}{p}\right\}\right\}\left\{\left\{v_{y}\right\}\right\} \llbracket v_{y} \rrbracket, \\
& \llbracket w_{2} \rrbracket=\llbracket \frac{\varrho}{p} v_{x} \rrbracket=\left\{\left\{\frac{\varrho}{p}\right\} \llbracket \llbracket v_{x} \rrbracket+\left\{\left\{v_{x}\right\}\right\} \llbracket \frac{\varrho}{p} \rrbracket,\right. \\
& \llbracket w_{3} \rrbracket=\llbracket \frac{\varrho}{p} v_{y} \rrbracket=\left\{\left\{\frac{\varrho}{p}\right\} \rrbracket \llbracket v_{y} \rrbracket+\left\{\left\{v_{y}\right\}\right\} \llbracket \frac{\varrho}{p} \rrbracket,\right. \\
& \llbracket w_{4} \rrbracket=-\llbracket \frac{\varrho}{p} \rrbracket, \\
& \llbracket \psi_{x} \rrbracket=\llbracket \varrho v_{x} \rrbracket=\{\{\varrho\}\} \llbracket v_{x} \rrbracket+\left\{\left\{v_{x}\right\}\right\} \llbracket \varrho, \\
& \llbracket \psi_{y} \rrbracket=\llbracket \varrho v_{y} \rrbracket=\{\{\varrho\}] \llbracket v_{y} \rrbracket+\left\{\left\{v_{y}\right\}\right\} \llbracket \varrho \rrbracket,
\end{aligned}
$$


the entropy conservation conditions $\llbracket w \rrbracket \cdot f^{\text {num, } x / y}-\llbracket \psi_{x / y} \rrbracket=0$ (33) become

$$
\begin{aligned}
& 0=\left(\frac{1}{\left\{\{\varrho\}_{\log }\right.} f_{\varrho}^{\text {num }, x}-\left\{\left\{v_{x}\right\}\right) \llbracket \varrho \rrbracket+\left(-\left\{\int \frac{\varrho}{p}\right\}\left\{\left\{v_{x}\right\}\right\} f_{\varrho}^{\text {num }, x}+\left\{\left\{\frac{\varrho}{p}\right\}\right\} f_{\varrho v_{x}}^{\text {num }, x}-\{\{\varrho\}\}\right) \llbracket v_{x} \rrbracket\right. \\
& +\left(-\left\{\left\{\frac{\varrho}{p}\right\}\right\}\left\{\left\{v_{y}\right\}\right\} f_{\varrho}^{\text {num }, x}+\left\{\left\{\frac{\varrho}{p}\right\}\right\} f_{\varrho}^{\text {num }, x}\right) \llbracket v_{y} \rrbracket \\
& +\left(\frac{1}{\gamma-1} \llbracket \log \frac{\varrho}{p} \rrbracket f_{\varrho}^{\text {num }, x}-\frac{\left\{\left\{v_{x}^{2}\right\}\right\}+\left\{\left\{v_{y}^{2}\right\}\right\}}{2} \llbracket \frac{\varrho}{p} \rrbracket f_{\varrho}^{\text {num }, x}+\left\{\left\{v_{x}\right\}\right] \llbracket \frac{\varrho}{p} \rrbracket f_{\varrho v_{x}}^{\text {num }, x}\right. \\
& +\left\{\left\{v_{y}\right\} \llbracket \llbracket \frac{\varrho}{p} \rrbracket f_{\varrho v_{y}}^{\text {num }, x}-\llbracket \frac{\varrho}{p} \rrbracket f_{\varrho e}^{\text {num }, x}\right), \\
& 0=\left(\frac{1}{\left\{\{\varrho\}_{\log }\right.} f_{\varrho}^{\text {num }, y}-\left\{\left\{v_{y}\right\}\right) \llbracket \varrho \rrbracket+\left(-\left\{\int \frac{\varrho}{p}\right\}\right\}\left\{\left\{v_{x}\right\} f_{\varrho}^{\text {num }, y}+\left\{\int \frac{\varrho}{p}\right\}\right\} f_{\varrho}^{\text {num }, y}\right) \llbracket v_{x} \rrbracket \\
& +\left(-\left\{\left\{\frac{\varrho}{p}\right\}\right\}\left\{\left\{v_{y}\right\}\right\} f_{\varrho}^{\text {num }, y}+\left\{\left\{\frac{\varrho}{p}\right\}\right\} f_{\varrho v_{y}}^{\text {num }, y}-\{\{\varrho\}) \llbracket v_{y} \rrbracket\right. \\
& +\left(\frac{1}{\gamma-1} \llbracket \log \frac{\varrho}{p} \rrbracket f_{\varrho}^{\text {num }, y}-\frac{\left\{\left\{v_{x}^{2}\right\}\right\}+\left\{\left\{v_{y}^{2}\right\}\right\}}{2} \llbracket \frac{\varrho}{p} \rrbracket f_{\varrho}^{\text {num }, y}+\left\{\left\{v_{x}\right\}\right] \llbracket \frac{\varrho}{p} \rrbracket f_{\varrho v_{x}}^{\text {num }, y}\right. \\
& +\left\{\left\{v_{y}\right\} \llbracket \llbracket \frac{\varrho}{p} \rrbracket f_{\varrho v_{y}}^{\text {num }, y}-\llbracket \frac{\varrho}{p} \rrbracket f_{\varrho e}^{\text {num }, y}\right) .
\end{aligned}
$$

Thus, the density and momentum fluxes

$$
\begin{aligned}
& f^{\text {num }, x}\left\{\begin{array}{l}
f_{\varrho}^{\text {num }, x}=\{\{\varrho\}\}_{\log }\left\{\left\{v_{x}\right\},\right. \\
f_{\varrho v_{x}}^{\text {num }, x}=\left\{\left\{v_{x}\right\}\right\} f_{\varrho}^{\text {num }, x}+\frac{\{\varrho \varrho\}}{\{\varrho \rho p\}}, \\
f_{\varrho v_{y}, x}^{\text {num }}=\left\{\left\{v_{y}\right\}\right\} f_{\varrho}^{\text {num }, x},
\end{array}\right. \\
& f^{\text {num }, y}\left\{\begin{array}{l}
f_{\varrho}^{\text {num }, y}=\{\{\varrho\}\}_{\log }\left\{\left\{v_{y}\right\},\right. \\
f_{\varrho v_{y}, y}^{\text {num }}=\left\{\left\{v_{y}\right\}\right\} f_{\varrho}^{\text {num }, x}, \\
f_{\varrho v_{y}, y}^{\text {num },}=\left\{\left\{v_{y}\right\}\right\} f_{\varrho}^{\text {num }, y}+\frac{\{\lfloor\varrho\}}{\{\lfloor\varrho / p\}},
\end{array}\right.
\end{aligned}
$$

set the two terms to zero. These fluxes are the same as in (52) and (75), i.e. the same as the ones used by Chandrashekar [3]. However, depending on the expression of $\llbracket \log \frac{\varrho}{p} \rrbracket$, different energy fluxes can be constructed, resulting in entropy conservative and kinetic energy preserving schemes.

Choosing $\chi=\beta \propto \frac{\varrho}{p}$, Chandrashekar [3] set $\llbracket \log \frac{\varrho}{p} \rrbracket=\frac{1}{\left\{\{\chi\}_{\log }\right.} \llbracket \chi \rrbracket$ and derived his EC and KEP flux (52). Choosing $\chi=R T=\left(\frac{\varrho}{p}\right)^{-1}$ and setting $\llbracket \log \frac{\underline{o}}{p} \rrbracket=-\frac{1}{\{\chi \chi\}_{\log }} \llbracket \chi \rrbracket$, the flux (75) has been derived in section 4.7 .

\subsubsection{Variant 1}

More generally, choosing $r \in \mathbb{R} \backslash\{0\}$, and setting $\frac{\varrho}{p}=\chi^{r}$, the jumps become

$$
\llbracket \log \frac{\varrho}{p} \rrbracket=\llbracket \log \chi^{r} \rrbracket=r \llbracket \log \chi \rrbracket=\frac{r}{\left\{\chi \chi \rrbracket_{\log }\right.} \llbracket \chi \rrbracket, \quad \llbracket \frac{\varrho}{p} \rrbracket=\llbracket \chi^{r} \rrbracket=\frac{\chi_{+}^{r}-\chi_{-}^{r}}{\chi_{+}-\chi_{-}} \llbracket \chi \rrbracket,
$$

where the mean value

$$
\left\{\{\chi\}_{x \mapsto x^{r}}:=\left(\frac{1}{r} \frac{\chi_{+}^{r}-\chi_{-}^{r}}{\chi_{+}-\chi_{-}}\right)^{1 /(r-1)}\right.
$$


can be introduced to yield

$$
\llbracket \chi^{r} \rrbracket=r\{\chi \chi\}_{x \mapsto x^{r}}^{r-1} \llbracket \chi \rrbracket .
$$

Thus, the arithmetic mean (36) becomes $\left\{\{a\}=\left\{\{a\}_{x \mapsto x^{2}}\right.\right.$ and the geometric mean (65) becomes $\left\{\{a\}_{\mathrm{geo}}=\left\{\{a\}_{x \mapsto 1 / x}\right.\right.$.

Using this mean value, entropy conservative, kinetic energy preserving, and consistent numerical fluxes are

$$
\begin{aligned}
& f^{\text {num }, x}\left\{\begin{array}{l}
f_{\varrho}^{\text {num }, x}=\{\{\varrho\}\}_{\log }\left\{\left\{v_{x}\right\},\right. \\
f_{\varrho v_{x}}^{\text {num }, x}=\left\{\left\{v_{x}\right\}\right\} f_{\varrho}^{\text {num }, x}+\frac{\{\lfloor\varrho\}}{\{\varrho \rho p\}\}}, \\
f_{\varrho v_{y}}^{\text {num }, x}=\left\{\left\{v_{y}\right\} f_{\varrho}^{\text {num }, x},\right. \\
f_{\varrho e}^{\text {num }, x}=\left(\frac{1}{\gamma-1} \frac{1}{\left\{\{\chi\}_{x \mapsto x^{r}}^{r-1}\{\{\chi\}\}_{l o g}\right.}-\frac{\left\{\left\{v_{x}^{2}\right\}\right\}+\left\{\left\{v_{y}^{2}\right\}\right\}}{2}\right) f_{\varrho}^{\text {num }, x}+\left\{\left\{v_{x}\right\}\right\} f_{\varrho v_{x}}^{\text {num }, x}+\left\{\left\{v_{y}\right\} f_{\varrho v_{y}}^{\text {num }, x},\right.
\end{array}\right. \\
& f^{\text {num }, y}\left\{\begin{array}{l}
f_{\varrho}^{\text {num }, y}=\{\{\varrho\}\}_{\log }\left\{\left\{v_{y}\right\}\right\}, \\
f_{\varrho v_{y}}^{\text {num }, y}=\left\{\left\{v_{y}\right\} f_{\varrho}^{\text {num }, x},\right. \\
f_{\varrho v_{y}, y}^{\text {num } y}=\left\{\left\{v_{y}\right\} f_{\varrho}^{\text {num }, y}+\frac{\{\{\varrho\}}{\{\{\varrho / p\}\}},\right. \\
f_{\varrho e}^{\text {num, }, y}=\left(\frac{1}{\gamma-1} \frac{1}{\left\{\{\chi\}_{x \mapsto x^{r}}^{r-1}\{\{\chi\}\}_{\log }\right.}-\frac{\left\{\left\{v_{x}^{2}\right\}\right\}+\left\{\left\{v_{y}^{2}\right\}\right\}}{2}\right) f_{\varrho}^{\text {num }, y}+\left\{\left\{v_{x}\right\}\right\} f_{\varrho v_{x}}^{\text {num }, y}+\left\{\left\{v_{y}\right\}\right\} f_{\varrho v_{y}}^{\text {num }, y} .
\end{array}\right.
\end{aligned}
$$

Of course, some numerically stable procedure to compute $\left\{\{\chi\}_{x \mapsto x^{r}}^{r-1}=\frac{1}{r} \frac{\chi_{+}^{r}-\chi_{-}^{r}}{\chi_{+}-\chi_{-}}\right.$has to be derived.

\subsubsection{Variant 2}

The choice $\frac{\varrho}{p}=\exp \chi$ results in

$$
\llbracket \log \frac{\varrho}{p} \rrbracket=\llbracket \log \exp \chi \rrbracket=\llbracket \chi \rrbracket, \quad \llbracket \frac{\varrho}{p} \rrbracket=\llbracket \exp \chi \rrbracket=\frac{\exp \chi_{+}-\exp \chi_{-}}{\chi_{+}-\chi_{-}} \llbracket \chi \rrbracket,
$$

where the mean value

$$
\left\{\{\chi\}_{x \mapsto \exp x}:=\log \frac{\exp \chi_{+}-\exp \chi_{-}}{\chi_{+}-\chi_{-}}\right.
$$

can be introduced to yield

$$
\llbracket \exp \chi \rrbracket=\exp \left(\left\{\chi \rrbracket_{x \mapsto \exp x}\right) \llbracket \chi \rrbracket .\right.
$$

Using this mean value, an entropy conservative, kinetic energy preserving, and consistent 
numerical flux is

$$
\begin{aligned}
& f^{\text {num }, x}\left\{\begin{array}{l}
f_{\varrho}^{\text {num }, x}=\left\{\{\varrho\}_{\log }\left\{\left\{v_{x}\right\}\right\},\right. \\
f_{\varrho v_{x}}^{\text {num } x}=\left\{\left\{v_{x}\right\} f_{\varrho}^{\text {num }, x}+\frac{\{\{\varrho\}\}}{\{\{\varrho / p\}\}},\right. \\
f_{\varrho v_{y}}^{\text {num }, x}=\left\{\left\{v_{y}\right\}\right\} f_{\varrho}^{\text {num }, x}, \\
f_{\varrho \varrho}^{\text {num }, x}=\left(\frac{1}{\gamma-1} \frac{1}{\exp \left\{\{\chi\}_{x \mapsto \exp x}\right.}-\frac{\left\{\left\{v_{x}^{2}\right\}\right]+\left\{\left\{v_{y}^{2}\right\}\right\}}{2}\right) f_{\varrho}^{\text {num }, x}+\left\{\left\{v_{x}\right\}\right\} f_{\varrho v_{x}}^{\text {num }, x}+\left\{\left\{v_{y}\right\}\right\} f_{\varrho v_{y}}^{\text {num }, x},
\end{array}\right. \\
& f^{\text {num }, y}\left\{\begin{array}{l}
f_{\varrho}^{\text {num }, y}=\left\{\{ \varrho \} _ { \operatorname { l o g } } \left\{\left\{v_{y}\right\},\right.\right. \\
f_{\varrho v_{y}}^{\text {num }, y}=\left\{\left\{v_{y}\right\} f_{\varrho}^{\text {num }, x},\right. \\
f_{\varrho v_{y}}^{\text {num }, y}=\left\{\left\{v_{y}\right\}\right\} f_{\varrho}^{\text {num }, y}+\frac{\{\{\varrho\}}{\{\{\varrho / p\}\}}, \\
f_{\varrho e}^{\text {num }, y}=\left(\frac{1}{\gamma-1} \frac{1}{\exp \left\{\{\chi\}_{x \mapsto \exp x}\right.}-\frac{\left.\left\{v_{x}^{2}\right\}\right\}+\left\{\left\{v_{y}^{2}\right\}\right\}}{2}\right) f_{\varrho}^{\text {num }, y}+\left\{\left\{v_{x}\right\}\right\} f_{\varrho v_{x}}^{\text {num }, y}+\left\{\left\{v_{y}\right\}\right\} f_{\varrho v_{y}}^{\text {num }, y} .
\end{array}\right.
\end{aligned}
$$

Again, some numerically stable procedure to compute $\exp \left\{\{\chi\}_{x \mapsto \exp x}=\frac{\exp \chi_{+}-\exp \chi_{-}}{\chi_{+}-\chi_{-}}\right.$has to be derived.

\subsection{Using Other Variables}

Of course, some other sets of variables can be used to derive entropy conservative numerical fluxes similar to the previous sections. However, since there is no clear intuition which choice of variables might be "good", this is not carried out in detail here. As noted by Derigs, Winters, Gassner, and Walch [6], an influence of the pressure in the numerical density flux should be avoided, see also Remark 6.2 and the numerical tests in section 7 .

\section{Reversing the Role of Energy and Entropy}

As proposed in [5] and used in [2, Section 2.4.6] to derive an approximate Riemann solver based on the Suliciu relaxation approach, the role of energy and entropy for the Euler equations can be reversed, i.e. a conservation law for the entropy and an inequality for the energy can be considered, cf. [7]. Then, the system reads

$$
\partial_{t} \underbrace{\left(\begin{array}{c}
\varrho \\
\varrho v_{x} \\
\varrho v_{y} \\
\varrho s
\end{array}\right)}_{=u}+\partial_{x} \underbrace{\left(\begin{array}{c}
\varrho v_{x} \\
\varrho v_{x}^{2}+p \\
\varrho v_{x} v_{y} \\
\varrho s v_{x}
\end{array}\right)}_{=f_{x}(u)}+\partial_{y} \underbrace{\left(\begin{array}{c}
\varrho v_{y} \\
\varrho v_{x} v_{y} \\
\varrho v_{y}^{2}+p \\
\varrho s v_{y}
\end{array}\right)}_{=f_{y}(u)}=0,
$$

and the 'entropy' condition becomes

$$
\partial_{t} \underbrace{(\varrho e)}_{=U}+\partial_{x} \underbrace{\left((\varrho e+p) v_{x}\right)}_{=F_{x}}+\partial_{y} \underbrace{\left((\varrho e+p) v_{y}\right)}_{=F_{y}} \leq 0 .
$$

Since smooth solutions satisfy (93) with equality, they are also smooth solutions of the Euler equations (1) with equality in the usual entropy condition (4). In the same spirit, an 'entropy' conserving numerical flux for (92), (93) is an entropy conserving flux for (1), (4) and vice versa.

Remark 5.1. This reversion of the energy and the entropy is very specific to the Euler equations. Exchanging some conserved quantity with the entropy will in general not result in a convex "entropy" with such nice properties as used here. 
In order to express the energy $\varrho e=\frac{1}{2} \varrho v^{2}+\varrho \varepsilon$ as a function of the new conserved variables $\varrho, \varrho v, \varrho s$, the pressure $p=(\gamma-1) \varrho \varepsilon$ can be inserted into the specific entropy $s=\log \frac{p}{\varrho^{\gamma}}$ to yield

$$
\varrho \varepsilon=\frac{\varrho^{\gamma}}{\gamma-1} \exp s
$$

Thus, the energy can be written as

$$
\varrho e=\frac{1}{2} \frac{(\varrho v)^{2}}{\varrho}+\frac{1}{\gamma-1} \varrho^{\gamma} \exp \left(\frac{\varrho s}{\varrho}\right) .
$$

Therefore, the new 'entropy' variables are

$$
\begin{aligned}
w=\frac{\partial(\varrho e)}{\partial\left(\varrho, \varrho v_{x}, \varrho v_{y}, \varrho s\right)}= & \left(\begin{array}{c}
-\frac{1}{2} \frac{(\varrho v)^{2}}{\varrho^{2}}+\frac{\gamma}{\gamma-1} \varrho^{\gamma-1} \exp \left(\frac{\underline{\varrho} s}{\varrho}\right)-\frac{1}{\gamma-1} \varrho^{\gamma} \frac{\underline{\varrho}}{\varrho^{2}} \exp \left(\frac{\varrho s}{\varrho}\right) \\
\frac{\varrho v_{x}}{\varrho} \\
\frac{\varrho v_{y}}{\varrho} \\
\frac{1}{\gamma-1} \varrho^{\gamma-1} \exp \left(\frac{\varrho s}{\varrho}\right) \\
=
\end{array}\right) \\
& \left(\begin{array}{c}
-\frac{1}{2} v^{2}+\frac{\gamma}{\gamma-1} \varrho^{\gamma-1} \exp (s)-\frac{1}{\gamma-1} \varrho^{\gamma-1} s \exp (s) \\
v_{x} \\
v_{y} \\
\frac{1}{\gamma-1} \varrho^{\gamma-1} \exp (s)
\end{array}\right) .
\end{aligned}
$$

The new 'entropy' fluxes are

$$
\begin{aligned}
& F_{x}=(\varrho e+p) v_{x}=\frac{1}{2} \varrho v^{2} v_{x}+\gamma \varrho \varepsilon v_{x}=\frac{1}{2} \varrho v^{2} v_{x}+\frac{\gamma}{\gamma-1} \varrho^{\gamma} v_{x} \exp (s), \\
& F_{y}=(\varrho e+p) v_{y}=\frac{1}{2} \varrho v^{2} v_{y}+\gamma \varrho \varepsilon v_{y}=\frac{1}{2} \varrho v^{2} v_{y}+\frac{\gamma}{\gamma-1} \varrho^{\gamma} v_{y} \exp (s),
\end{aligned}
$$

so that the new flux potentials $\psi_{x / y}$ fulfilling $\partial_{w} \psi_{x / y}=f_{x / y}(u(w))$ become

$$
\begin{aligned}
\psi_{x / y}=w \cdot f_{x / y}-F_{x / y}= & \left(-\frac{1}{2} v^{2}+\frac{\gamma}{\gamma-1} \varrho^{\gamma-1} \exp (s)-\frac{1}{\gamma-1} \varrho^{\gamma-1} s \exp (s)\right) \varrho v_{x / y} \\
& +v_{x / y}\left(\varrho v_{x / y}^{2}+\varrho^{\gamma} \exp (s)\right)+v_{y / x} \varrho v_{x / y} v_{y / x} \\
& +\frac{1}{\gamma-1} \varrho^{\gamma-1} \exp (s) \varrho s v_{x / y}-\frac{1}{2} \varrho v^{2} v_{x / y}-\frac{\gamma}{\gamma-1} \varrho^{\gamma} v_{x / y} \exp (s) \\
= & \varrho^{\gamma} v_{x / y} \exp (s) .
\end{aligned}
$$

As before, the conditions for 'entropy' conservation in the semidiscrete setting of Tadmor [25, 26] are $\llbracket w \rrbracket \cdot f^{\text {num }, x / y}-\llbracket \psi_{x / y} \rrbracket=0(33)$. The corresponding 'entropy' fluxes are $F_{x / y}^{\text {num }}=\{\{w\}\}$. $f^{\text {num }, x / y}-\left\{\left\{\psi_{x / y}\right\}\right\}$, i.e. the numerical energy fluxes corresponding to an 'entropy' conservative flux $f^{\text {num }, x / y}$ for (92) are

$$
f_{\varrho e}^{\text {num } x / y}=\{\{w\}\} \cdot f^{\text {num }, x / y}-\left\{\left\{\psi_{x / y}\right\},\right.
$$

with $w$ as in (96) and $\psi_{x / y}$ as in (98).

Choosing $v$ as variable and writing the jumps using the product rule (37) as

$$
\begin{aligned}
& \llbracket w_{1} \rrbracket=-\left\{v_{x}\right\} \llbracket v_{x} \rrbracket-\left\{v_{y}\right\} \llbracket \llbracket v_{y} \rrbracket+\frac{\gamma}{\gamma-1} \llbracket \varrho^{\gamma-1} \exp (s) \rrbracket-\frac{1}{\gamma-1} \llbracket \varrho^{\gamma-1} s \exp (s) \rrbracket, \\
& \llbracket w_{2} \rrbracket=\llbracket v_{x} \rrbracket, \\
& \llbracket w_{3} \rrbracket=\llbracket v_{y} \rrbracket, \\
& \llbracket w_{4} \rrbracket=\frac{1}{\gamma-1} \llbracket \varrho^{\gamma-1} \exp (s) \rrbracket, \\
& \llbracket \psi_{x} \rrbracket=\left\{\left\{\varrho^{\gamma} \exp (s)\right\} \llbracket \llbracket v_{x} \rrbracket+\left\{\left\{v_{x}\right\} \llbracket \llbracket \varrho^{\gamma} \exp (s) \rrbracket,\right.\right. \\
& \llbracket \psi_{y} \rrbracket=\left\{\left\{\varrho^{\gamma} \exp (s)\right\} \llbracket v_{y} \rrbracket+\left\{\left\{v_{y}\right\} \llbracket \llbracket \varrho^{\gamma} \exp (s) \rrbracket,\right.\right.
\end{aligned}
$$


the coefficients of $\llbracket v_{x / y} \rrbracket$ in the entropy conditions $\llbracket w \rrbracket \cdot f^{\text {num }, x / y}-\llbracket \psi_{x / y} \rrbracket=0$ become

$$
-\left\{\left\{v_{x}\right\}\right\} f_{\varrho}^{\text {num, } x}+f_{\varrho v_{x}}^{\text {num }, x}-\left\{\left\{\varrho^{\gamma} \exp (s)\right\}, \quad-\left\{\left\{v_{y}\right\}\right\} f_{\varrho}^{\text {num }, y}+f_{\varrho v_{y}}^{\text {num }, y}-\left\{\left\{\varrho^{\gamma} \exp (s)\right\}\right\} .\right.
$$

Thus, general momentum fluxes for entropy conservative numerical fluxes can be written as

$$
\begin{aligned}
f^{\text {num }, x}\left\{\begin{array}{l}
f_{\varrho v_{x}}^{\text {num }, x}=\left\{\left\{v_{x}\right\} f_{\varrho}^{\text {num }, x}+\left\{\left\{\varrho^{\gamma} \exp (s)\right\}\right\}=\left\{\left\{v_{x}\right\}\right\} f_{\varrho}^{\text {num }, x}+\{\{p\},\right. \\
f_{\varrho v_{y}}^{\text {num }, x}=\left\{\left\{v_{y}\right\}\right\} f_{\varrho}^{\text {num }, x},
\end{array}\right. \\
f^{\text {num, }, y}\left\{\begin{array}{l}
f_{\varrho v_{x}, y}^{\text {num }}=\left\{\left\{v_{x}\right\} f_{\varrho}^{\text {num }, y},\right. \\
f_{\varrho v_{y}}^{\text {num }, y}=\left\{\left\{v_{y}\right\}\right\} f_{\varrho}^{\text {num }, y}+\left\{\left\{\varrho^{\gamma} \exp (s)\right\}\right\}=\left\{\left\{v_{y}\right\}\right\} f_{\varrho}^{\text {num }, y}+\{\{p\},
\end{array}\right.
\end{aligned}
$$

i.e. in the form proposed by Jameson [16] for a kinetic energy preserving flux (in one space dimension). Another possibility would be to split the jump of $\psi_{x / y}$ in some other way, resulting in a numerical pressure flux $p^{\text {num }}$ different from $\{\{p\}$.

\subsection{Using $\varrho, v, T$ as Variables}

Using the variables $\varrho, v$, and $R T=\frac{p}{\varrho}=\varrho^{\gamma-1} \exp (s)$, the flux potentials $\psi_{x / y}=\varrho^{\gamma} v_{x / y} \exp (s)$ (98) and the entropy variables (96) can be written as

$$
\psi_{x / y}=\varrho R T v_{x / y}, \quad w=\left(\begin{array}{c}
-\frac{1}{2} v^{2}+\frac{\gamma}{\gamma-1} R T-\frac{1}{\gamma-1} s R T \\
v_{x} \\
v_{y} \\
\frac{1}{\gamma-1} R T
\end{array}\right), \quad s=\log R T-(\gamma-1) \log \varrho .
$$

\subsubsection{Variant 1}

Using these variables, the jumps can be written using the chain rules (37) and (39) as

$$
\begin{aligned}
\llbracket w_{1} \rrbracket= & -\frac{1}{2} \llbracket v^{2} \rrbracket+\frac{\gamma}{\gamma-1} \llbracket R T \rrbracket-\frac{1}{\gamma-1} \llbracket R T \log R T \rrbracket+\llbracket R T \log \varrho \rrbracket \\
= & -\left\{\left\{v_{x} \rrbracket \llbracket \llbracket v_{x} \rrbracket-\left\{\left\{v_{y}\right\}\right\} \llbracket v_{y} \rrbracket+\frac{\gamma}{\gamma-1} \llbracket R T \rrbracket-\frac{1}{\gamma-1} \frac{\{\{R T\}]}{\left\{\{R T\}_{\log }\right.} \llbracket R T \rrbracket\right.\right. \\
& -\frac{1}{\gamma-1}\{\{\log R T\}\} \llbracket R T \rrbracket+\{\{\log \varrho\}\} \llbracket R T \rrbracket+\frac{\{\{R T\}}{\left\{\{\varrho\}_{\log }\right.} \llbracket \varrho \rrbracket, \\
\llbracket w_{2} \rrbracket= & \llbracket v_{x} \rrbracket \\
\llbracket w_{3} \rrbracket= & \llbracket v_{y} \rrbracket, \\
\llbracket w_{4} \rrbracket= & \frac{1}{\gamma-1} \llbracket R T \rrbracket, \\
\llbracket \psi_{x} \rrbracket= & \left\{\{\varrho R T\} \llbracket \llbracket v_{x} \rrbracket+\left\{\left\{v_{x}\right\}\right\} \llbracket \varrho R T \rrbracket=\{\{\varrho R T\}\} \llbracket v_{x} \rrbracket+\{\{\varrho\}\}\left\{\left\{v_{x}\right\} \rrbracket \llbracket R T \rrbracket+\left\{\left\{v_{x}\right\}\right\}\{\{R T\} \llbracket \varrho \rrbracket,\right.\right. \\
\llbracket \psi_{y} \rrbracket= & \left\{\{\varrho R T\} \llbracket \llbracket v_{y} \rrbracket+\left\{\left\{v_{y}\right\}\right\} \llbracket \varrho R T \rrbracket=\left\{\{\varrho R T\} \llbracket \llbracket v_{y} \rrbracket+\{\{\varrho\}\}\left\{v_{y}\right\}\right\} \llbracket R T \rrbracket+\left\{\left\{v_{y}\right\}\right\}\{\{R T\} \llbracket \varrho \rrbracket .\right.
\end{aligned}
$$


Inserting this in the entropy conditions $\llbracket w \rrbracket \cdot f^{\text {num }, x / y}-\llbracket \psi_{x / y} \rrbracket=0$,

$$
\begin{aligned}
0= & \left(\frac{\{R T\}}{\left\{\lfloor\varrho\}_{\log }\right.} f_{\varrho}^{\text {num, } x}-\left\{\left\{v_{x}\right\}\right\}\{\{R T\}) \llbracket \varrho \rrbracket\right. \\
& +\left(-\left\{\left\{v_{x}\right\}\right\} f_{\varrho}^{\text {num }, x}+f_{\varrho v_{x}}^{\text {num }, x}-\{\{\varrho R T\}) \llbracket v_{x} \rrbracket+\left(-\left\{\left\{v_{y}\right\}\right\} f_{\varrho}^{\text {num }, x}+f_{\varrho v_{y}}^{\text {num } x}\right) \llbracket v_{y} \rrbracket\right. \\
& +\left(\left(\frac{\gamma}{\gamma-1}-\frac{1}{\gamma-1} \frac{\{\{R T\}}{\left\{\{R T\}_{\log }\right.}-\frac{1}{\gamma-1}\left\{\{\log R T\}+\{\{\log \varrho\}) f_{\varrho}^{\text {num }, x}\right.\right.\right. \\
& +\frac{1}{\gamma-1} f_{\varrho s}^{\text {num }, x}-\left\{\{\varrho\}\left\{\left\{v_{x}\right\}\right\}\right) \llbracket R T \rrbracket, \\
0= & \frac{\{\{R T\}}{\left\{\{\varrho\}_{\log }\right.} f_{\varrho}^{\text {num, } y}-\left\{\left\{v_{y}\right\}\right\}\{\{R T\}) \llbracket \varrho \rrbracket \\
& +\left(-\left\{\left\{v_{x}\right\} f_{\varrho}^{\text {num }, y}+f_{\varrho v_{x}}^{\text {num }, y}\right) \llbracket v_{x} \rrbracket+\left(-\left\{\left\{v_{y}\right\}\right\} f_{\varrho}^{\text {num }, y}+f_{\varrho v_{y}}^{\text {num }, y}-\{\{\varrho R T\}) \llbracket v_{y} \rrbracket\right.\right. \\
& +\left(\left(\frac{\gamma}{\gamma-1}-\frac{1}{\gamma-1} \frac{\{\{R T\}\}}{\{\{R T\}\}_{\log }}-\frac{1}{\gamma-1}\left\{\{\log R T\}+\{\{\log \varrho\}) f_{\varrho}^{\text {num }, y}\right.\right.\right. \\
& \quad+\frac{1}{\gamma-1} f_{\varrho s}^{\text {num }, y}-\left\{\{\varrho\}\left\{\left\{v_{y}\right\}\right\}\right) \llbracket R T \rrbracket,
\end{aligned}
$$

the fluxes

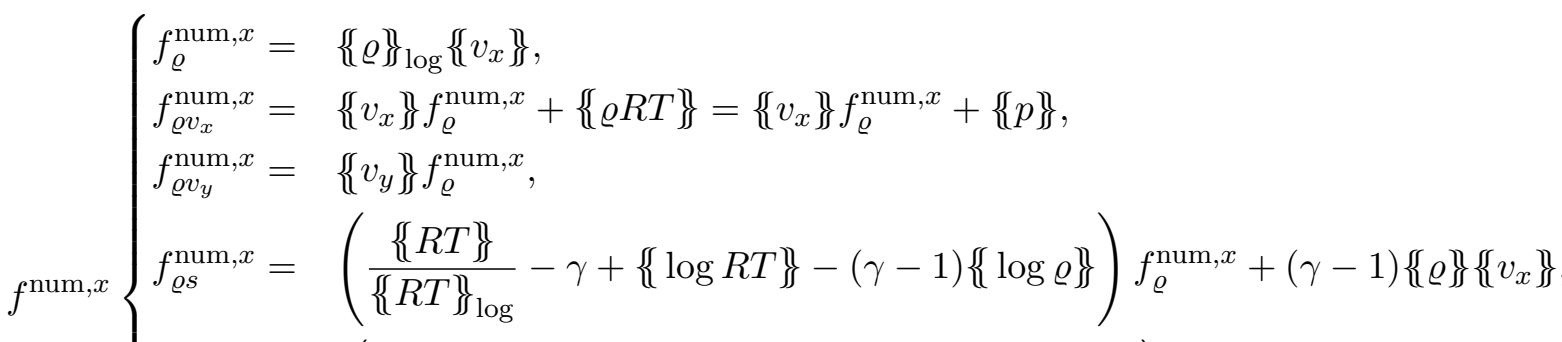

$$
\begin{aligned}
& f_{\varrho e}^{\text {num }, x}=\left(-\frac{\left.\left\{v_{x}^{2}\right\}\right\}+\left\{\left\{v_{y}^{2}\right\}\right\}}{2}+\frac{\gamma}{\gamma-1}\left\{\{R T\}-\frac{1}{\gamma-1}\{\{s R T\}) f_{\varrho}^{\text {num }, x}\right.\right. \\
& +\left\{\left\{v_{x}\right\} f_{\varrho v_{x}}^{\text {num }, x}+\left\{\left\{v_{y}\right\}\right\} f_{\varrho v_{y}}^{\text {num }, x}+\frac{1}{\gamma-1}\{\{R T\}\} f_{\varrho s}^{\text {num }, x}-\left\{\left\{\varrho R T v_{x}\right\}\right\},\right. \\
& \left\{\begin{array}{l}
f_{\varrho}^{\text {num }, y}=\{\{\varrho\}\}_{\log }\left\{\left\{v_{y}\right\},\right. \\
f_{\varrho v_{x}, y}^{\text {num }, y}=\left\{\left\{v_{x}\right\} f_{\varrho}^{\text {num, }, y},\right. \\
f_{\varrho v_{y}}^{\text {num }, y}=\left\{\left\{v_{y}\right\} f_{\varrho}^{\text {num }, y}+\{\{\varrho R T\}\}=\left\{\left\{v_{y}\right\}\right\} f_{\varrho}^{\text {num }, y}+\{\{p\}\}\right.
\end{array}\right. \\
& f^{\text {num }, y}\left\{f_{\varrho s}^{\text {num }, y}=\left(\frac{\{\{R T\}}{\left\{\{R T\}_{\log }\right.}-\gamma+\left\{\{\log R T\}-(\gamma-1)\{\{\log \varrho\}) f_{\varrho}^{\text {num }, y}+(\gamma-1)\left\{\{\varrho\}\left\{\left\{v_{y}\right\}\right\},\right.\right.\right.\right. \\
& f_{\varrho e}^{\text {num }, y}=\left(-\frac{\left.\left\{v_{x}^{2}\right\}\right\}+\left\{\left\{v_{y}^{2}\right\}\right\}}{2}+\frac{\gamma}{\gamma-1}\left\{\{R T\}-\frac{1}{\gamma-1}\{\{s R T\}) f_{\varrho}^{\text {num }, y}\right.\right. \\
& +\left\{\left\{v_{x}\right\}\right\} f_{\varrho v_{x}}^{\text {num }, y}+\left\{\left\{v_{y}\right\}\right\} f_{\varrho v_{y}, y}^{\text {num }, y}+\frac{1}{\gamma-1}\{\{R T\}\} f_{\varrho s}^{\text {num }, y}-\left\{\left\{\varrho R T v_{y}\right\}\right\},
\end{aligned}
$$

can be seen to be consistent, entropy conservative, and kinetic energy preserving fluxes for the Euler equations (1), where the energy fluxes $f_{\varrho e}^{\text {num, } x / y}$ have been computed as $\{\{w\}\} \cdot f^{\text {num, } x / y}-$ $\left\{\left\{\psi_{x / y}\right\}\right.$. 
Inserting the numerical fluxes into the definition of the energy fluxes $f_{\varrho e}^{\text {num, } x}, f_{\varrho e}^{\text {num,y } y}$ yields

$$
\begin{aligned}
& f_{\varrho e}^{\text {num }, x}=\left(-\frac{\left\{\left\{v_{x}^{2}\right\}\right\}+\left\{\left\{v_{y}^{2}\right\}\right\}}{2}+\frac{\gamma}{\gamma-1}\left\{\{R T\}-\frac{1}{\gamma-1}\{\{s R T\})\left\{\{\varrho\}_{\log }\left\{\left\{v_{x}\right\}\right\}\right.\right.\right. \\
& +\left\{\left\{v_{x}\right\}\right\}\left(\left\{\{ \varrho \} _ { \operatorname { l o g } } \left\{\left\{v_{x}\right\}^{2}+\{\{\varrho R T\})+\left\{\left\{v_{y}\right\}\right\}\left(\left\{\{\varrho\}_{\log }\left\{\left\{v_{x}\right\}\right\}\left\{\left\{v_{y}\right\}\right\}\right)\right.\right.\right.\right. \\
& +\frac{1}{\gamma-1}\left\{\{ R T \} \left(\frac{\{\{R T\}}{\left\{\{R T\}_{\log }\right.}-\gamma+\{\{\log R T\}\}-(\gamma-1)\{\{\log \varrho\})\left\{\{\varrho\}_{\log }\left\{\left\{v_{x}\right\}\right\}\right.\right.\right. \\
& +\left\{\{R T\}\{\{\varrho\}\}\left\{\left\{v_{x}\right\}\right\}-\left\{\left\{\varrho v_{x} R T\right\}\right.\right. \\
& =\left\{\{\varrho\}_{\log }\left\{\left\{v_{x}\right\}\right\}\left(\left\{v_{x}\right\}^{2}+\left\{\left\{v_{y}\right\}\right\}^{2}-\frac{\left\{\left\{v_{x}^{2}\right\}\right\}+\left\{\left\{v_{y}^{2}\right\}\right\}}{2}\right)\right. \\
& \left.+\{\{\varrho\}\}\left\{v_{x}\right\}\right\}\{\{R T\}\}+\left\{\{\varrho R T\}\left\{\left\{v_{x}\right\}\right\}-\left\{\left\{\varrho v_{x} R T\right\}+\frac{1}{\gamma-1}\left\{\{\varrho\}_{\log }\left\{\left\{v_{x}\right\}\right\} \frac{\{\{R T\}\}^{2}}{\{\{R T\}\}_{\log }}\right.\right.\right. \\
& +\left\{\{ \varrho \} _ { \operatorname { l o g } } \{ \{ v _ { x } \} \} \left(\frac { 1 } { \gamma - 1 } \{ \{ R T \} \} \left\{\{\log R T\}-\left\{\{ \operatorname { l o g } \varrho \} \left\{\{R T\}-\frac{1}{\gamma-1}\{\{s R T\}),\right.\right.\right.\right.\right. \\
& f_{\varrho e}^{\text {num }, y}=\left(-\frac{\left\{\left\{v_{x}^{2}\right\}\right\}+\left\{\left\{v_{y}^{2}\right\}\right\}}{2}+\frac{\gamma}{\gamma-1}\left\{\{R T\}-\frac{1}{\gamma-1}\{\{s R T\})\{\{\varrho\}\}_{\log }\left\{\left\{v_{y}\right\}\right\}\right.\right. \\
& +\left\{\left\{v_{x}\right\}\right\}\left(\left\{\{\varrho\}_{\log }\left\{\left\{v_{x}\right\}\right\}\left\{\left\{v_{y}\right\}\right\}\right)+\left\{\left\{v_{y}\right\}\right\}\left(\{\{\varrho\}\}_{\log }\left\{\left\{v_{y}\right\}\right\}^{2}+\{\{\varrho R T\})\right.\right. \\
& +\frac{1}{\gamma-1}\left\{\{ R T \} \left(\frac{\{\{R T\}}{\left\{\{R T\}_{\log }\right.}-\gamma+\{\{\log R T\}\}-(\gamma-1)\{\{\log \varrho\})\left\{\{\varrho\}_{\log }\left\{\left\{v_{y}\right\}\right\}\right.\right.\right. \\
& +\left\{\{R T\}\{\{\varrho\}\}\left\{\left\{v_{y}\right\}\right\}-\left\{\left\{\varrho v_{y} R T\right\}\right\}\right. \\
& =\left\{\{ \varrho \} _ { \operatorname { l o g } } \{ \{ v _ { y } \} \} \left(\left\{\left\{v_{x}\right\}^{2}+\left\{\left\{v_{y}\right\}\right\}^{2}-\frac{\left\{\left\{v_{x}^{2}\right\}\right\}+\left\{\left\{v_{y}^{2}\right\}\right\}}{2}\right)\right.\right. \\
& +\left\{\{ \varrho \} \{ \{ v _ { y } \} \} \left\{\{R T\}+\left\{\{\varrho R T\}\left\{\left\{v_{y}\right\}\right\}-\left\{\left\{\varrho v_{y} R T\right\}+\frac{1}{\gamma-1}\left\{\{\varrho\}_{\log }\left\{\left\{v_{y}\right\}\right\} \frac{\left\{\{R T\}^{2}\right.}{\left\{\{R T\}_{\log }\right.}\right.\right.\right.\right.\right. \\
& +\{\{\varrho\}\}_{\log }\left\{\left\{v_{y}\right\}\right\}\left(\frac{1}{\gamma-1}\{\{R T\}\}\{\log R T\}-\left\{\{ \operatorname { l o g } \varrho \} \left\{\{R T\}-\frac{1}{\gamma-1}\{\{s R T\}) .\right.\right.\right.
\end{aligned}
$$

Here, the first two lines of the results are consistent approximations of the fluxes $\frac{1}{2} \varrho v^{2} v_{x / y}+\frac{\gamma}{\gamma-1} p$ with additional terms that are consistent with zero, since $s=\log R T-(\gamma-1) \log \varrho$.

\subsection{Other Variables}

As in section 4 , other choices of variables are possible, e.g. $\varrho, v, \chi$ with $\frac{p}{\varrho}=g(\chi)$. However, this approach is not further pursued here, since there does not seem to be a clear intuition, which choice is preferable.

\section{Numerical Surface Fluxes / Riemann Solvers}

The numerical fluxes $f^{\text {num }}$ used in the surface terms (12) of the semidiscretisation (10) are an important ingredient for stability and robustness of the method. In a first order finite volume setting, they determine the method completely. Here, some choices of these numerical fluxes will be presented and compared. Since they are used in the surface terms, they are frequently called surface fluxes in order to distinguish them from the volume fluxes $f^{\text {vol }}$ used in the volume terms (11) of the semidiscretisation (10). 


\subsection{Adding Dissipation to Entropy Conservative Fluxes}

Similarly to the local Lax-Friedrichs flux

$$
f_{\mathrm{LLF}}^{\mathrm{num}}\left(u_{-}, u_{+}\right)=\frac{f\left(u_{+}\right)+f\left(u_{-}\right)}{2}-\frac{\lambda}{2}\left(u_{+}-u_{-}\right),
$$

an entropy stable flux can be constructed as an entropy conservative central flux plus an additional dissipation term.

The simplest choice is to add a local Lax-Friedrichs type dissipation of the form $-\frac{\lambda}{2} \llbracket u \rrbracket$. The resulting flux is entropy stable, since multiplication with the jump of the entropy variables results in

$$
\begin{aligned}
-\frac{\lambda}{2} \llbracket w \rrbracket \cdot \llbracket u \rrbracket & =-\frac{\lambda}{2} \llbracket w \rrbracket \cdot \int_{0}^{1} \frac{\partial u}{\partial w}\left(w_{-}+\sigma\left(w_{+}-w_{-}\right)\right) \cdot\left(w_{+}-w_{-}\right) \mathrm{d} \sigma \\
& =-\frac{\lambda}{2} \llbracket w \rrbracket \cdot \int_{0}^{1} \frac{\partial u}{\partial w}\left(w_{-}+\sigma\left(w_{+}-w_{-}\right)\right) \mathrm{d} \sigma \cdot \llbracket w \rrbracket,
\end{aligned}
$$

and $\frac{\partial u}{\partial w}=\left(\frac{\partial w}{\partial u}\right)^{-1}$ is positive definite, since $w=\partial_{u} U$ and the entropy $U$ is convex.

Another construction uses a dissipation term of the form $-\frac{1}{2}\left|f^{\prime}(u)\right| \llbracket u \rrbracket \approx-\frac{1}{2}\left|f^{\prime}(u)\right| \partial_{w} u \cdot \llbracket w \rrbracket$. Using the scaling of the eigenvectors proposed by Barth [1, Theorem 4] results in $\left|f^{\prime}(u)\right|=$ $R|\Lambda| R^{-1}$ and $\partial_{w} u=R R^{T}$, where $\Lambda$ contains the eigenvalues of $f^{\prime}(u)$ on the diagonal. Thus, $-\left|f^{\prime}(u)\right| \llbracket u \rrbracket \approx-R|\Lambda| R^{T} \cdot \llbracket w \rrbracket$ and the matrix $R|\Lambda| R^{T}$ is positive definite.

Using this form, choosing $|\Lambda|=\lambda \mathrm{I}$ and some intermediate value $H=R R^{T}=\partial_{w} u$ results in a scalar dissipation term (SD) $-\frac{\lambda}{2} H \llbracket w \rrbracket$. A matrix dissipation term (MD) is obtained by setting $|\Lambda|=\operatorname{diag}\left(\left|\lambda_{i}\right|\right)$.

Of course, the matrices $H, R,|\Lambda|$ have to be evaluated at some suitable intermediate values. Derigs et al. [6, 28] investigated this problem for ideal MHD and the Euler equations using the entropy conservative flux (52) of [3] and derived scalar and matrix dissipation operators of the forms described above. Additionally, they proposed to use the convex combination $-\Xi \frac{\lambda}{2} H \llbracket w \rrbracket-(1-\Xi) \frac{1}{2} R|\Lambda| R^{T} \cdot \llbracket w \rrbracket$, where $\Xi=\left|\frac{p_{+}-p_{-}}{p_{+}+p_{-}}\right|^{\frac{1}{2}}$ is the indicator of the shock strength also used in [3].

\subsection{Preserving Positivity of the Density}

The Euler equations are valid for positive density $\varrho$ and pressure $p$. In order to be robust, the numerical flux $f^{\text {num }}$ should preserve these invariant regions of the Euler equations in a first order finite volume update procedure using an explicit Euler step in time

$$
u_{i}^{+}=u_{i}-\frac{\Delta t}{\Delta x}\left(f^{\text {num }}\left(u_{i}, u_{i+1}\right)-f^{\text {num }}\left(u_{i-1}, u_{i}\right)\right) .
$$

Extensions of this property to higher order methods can be constructed using the framework of Zhang and Shu [30]. As described inter alia in [31, Remark 2.4], the (local) Lax-Friedrichs flux preserves positivity of both density and pressure. Here, the entropy conservative numerical fluxes described in sections 4 and 5 are investigated. The main result concerning positivity of the density is

Theorem 6.1. Suppose that the numerical density flux $f_{\varrho}^{\text {num }}$ can be written as $f_{\varrho}^{\text {num }}=\bar{\varrho} \cdot\{\{v\}-$ $\frac{\lambda}{2} \llbracket \varrho \rrbracket$, where $\lambda \geq \max \left\{\left|v_{i}\right|,\left|v_{i+1}\right|\right\}$ and $\varrho$ is some mean value satisfying $\varrho \leq\{\{\varrho\}$, i.e.

$$
\bar{\varrho}\left(\varrho_{i}, \varrho_{i+1}\right) \in\left[\min \left\{\varrho_{i}, \varrho_{i+1}\right\}, \max \left\{\varrho_{i}, \varrho_{i+1}\right\}\right], \bar{\varrho}\left(\varrho_{i}, \varrho_{i+1}\right) \leq \frac{\varrho_{i}+\varrho_{i+1}}{2} .
$$

Then the first order FV scheme (110) preserves the non-negativity of the density @ under the CFL condition

$$
\Delta t \leq \frac{\Delta x}{2 \lambda}
$$


Remark 6.1. Note that the CFL condition (112) does not depend explicitly on the densities and that it does not require a vanishing time step $\Delta t$ for $\varrho \rightarrow 0$.

Corollary 6.1. If the numerical density flux $f_{\varrho}^{\text {num }}=\left\{\{\varrho\}_{\log }\{v\}\right\}-\frac{\lambda}{2} \llbracket \varrho \rrbracket$ is used with $\lambda \geq$ $\max \left\{\left|v_{i}\right|,\left|v_{i+1}\right|\right\}$, the first order FV scheme (110) preserves the non-negativity of the density $\varrho$ under the CFL condition (112).

Proof of Corollary 6.1. The logarithmic mean fulfils the conditions of Theorem 6.1, especially $\left\{\{\varrho\}_{\log } \leq\{\{\varrho\}\}[4]\right.$.

Remark 6.2. Due to Corollary 6.1, if the entropy conservative fluxes described in sections 4 and 5 containing no contribution of the pressure in the density flux are used with dissipation of LLF type in the variable $\varrho$, the first order FV scheme (110) preserves the non-negativity of the density $\varrho$ under the CFL condition (112). This is also fulfilled for the scalar dissipation operator SD of [6]. Contrary, the entropy conservative fluxes containing an influence of the pressure in the density flux do not fulfil the conditions of Theorem 6.1. This agrees with physical intuition, since the pressure has no influence on the density flux in the Euler equations (1) [6].

Proof of Theorem 6.1. The FV step (110) for the density can be separated into two parts as

$$
\varrho_{i}^{+}=\left(\frac{1}{2} \varrho_{i}-\frac{\Delta t}{\Delta x} f_{\varrho}^{\text {num }}\left(u_{i}, u_{i+1}\right)\right)+\left(\frac{1}{2} \varrho_{i}+\frac{\Delta t}{\Delta x} f_{\varrho}^{\text {num }}\left(u_{i-1}, u_{i}\right)\right) .
$$

Since both can be handled similarly, only the first one will be analysed. Inserting the numerical density flux, the mean value $\bar{\varrho}$ can be written as a convex combination $\bar{\varrho}=\alpha \varrho_{i}+(1-\alpha) \varrho_{i+1}$, $\alpha \in[0,1]$. Thus, the first term becomes

$$
\begin{aligned}
& \frac{1}{2} \varrho_{i}-\frac{\Delta t}{\Delta x}\left(\bar{\varrho}\left\{\{v\}-\frac{\lambda}{2} \llbracket \varrho \rrbracket\right)\right. \\
= & \varrho_{i}\left(\frac{1}{2}-\frac{\lambda}{2} \frac{\Delta t}{\Delta x}-\alpha\left\{\{v\} \frac{\Delta t}{\Delta x}\right)+\varrho_{i+1} \frac{\Delta t}{\Delta x}\left(\frac{\lambda}{2}-(1-\alpha)\{\{v\}\}\right) .\right.
\end{aligned}
$$

Using $\varrho_{i}, \varrho_{i+1} \geq 0$ and $\lambda \geq \max \left\{\left|v_{i}\right|,\left|v_{i+1}\right|\right\}$,

$$
\frac{1}{2} \varrho_{i}-\frac{\Delta t}{\Delta x}\left(\bar{\varrho}\left\{\{v\}-\frac{\lambda}{2} \llbracket \varrho \rrbracket\right) \geq \varrho_{i}\left(\frac{1}{2}-\left(\frac{1}{2}+\alpha\right) \frac{\lambda \Delta t}{\Delta x}\right)+\varrho_{i+1} \frac{\lambda \Delta t}{\Delta x}\left(\alpha-\frac{1}{2}\right) .\right.
$$

Two cases can be considered.

1. $\varrho_{i} \geq \varrho_{i+1}$. In this case, $\alpha \leq \frac{1}{2}$, since $\bar{\varrho} \leq\{\{\varrho\}$. Thus, the second term on the right hand side of (115) can be bounded as

$$
\varrho_{i+1} \frac{\lambda \Delta t}{\Delta x}\left(\alpha-\frac{1}{2}\right) \geq \varrho_{i} \frac{\lambda \Delta t}{\Delta x}\left(\alpha-\frac{1}{2}\right) .
$$

2. $\varrho_{i} \leq \varrho_{i+1}$. In this case, $\alpha \geq \frac{1}{2}$, since $\bar{\varrho} \leq\{\{\varrho\}$. Again, the second term on the right hand side of (115) can be bounded via (116).

In both cases, the term with $\varrho_{i+1}$ in $(115)$ can be estimated via $\varrho_{i}$, yielding

$$
\begin{aligned}
\frac{1}{2} \varrho_{i}-\frac{\Delta t}{\Delta x}\left(\bar{\varrho}\{v\}-\frac{\lambda}{2} \llbracket \varrho \rrbracket\right) & \geq \varrho_{i}\left(\frac{1}{2}-\left(\frac{1}{2}+\alpha\right) \frac{\lambda \Delta t}{\Delta x}\right)+\varrho_{i} \frac{\lambda \Delta t}{\Delta x}\left(\alpha-\frac{1}{2}\right) \\
& =\varrho_{i}\left(\frac{1}{2}-\frac{\lambda \Delta t}{\Delta x}\right) .
\end{aligned}
$$

This is non-negative under the CFL condition (112). 


\subsection{Preserving Positivity of the Pressure}

Preserving the positivity of the pressure / internal energy is more complicated than the corresponding property of the density. For the (local) Lax-Friedrichs flux, it can be proven as described inter alia in [31, Remark 2.4]. Further investigations have to be conducted for the case of the numerical fluxes considered here.

As a general procedure, the reversed roles of entropy and energy as in section 5 can be used to get entropy stable fluxes that preserve the positivity of the internal energy as described by Bouchut [2]. This corresponds to a computation of the pressure via the entropy, which has also been used in [7] in an a posteriori manner. However, this direction of further research will not be pursued here.

\subsection{Suliciu Relaxation Solver}

The Suliciu relaxation solver described in [2, Section 2.4] for the two-dimensional Euler equations in $x$ direction can be summed up as follows. At first, intermediate wave speeds are computed via

$$
\begin{aligned}
& \text { if } p_{+} \geq p_{-},\left\{\begin{array}{l}
\frac{c_{-}}{\varrho_{-}}=\sqrt{\gamma \frac{p_{-}}{\varrho_{-}}}+\frac{\gamma+1}{2} \max \left\{\frac{p_{+}-p_{-}}{\varrho_{+} \sqrt{\gamma p_{+} / \varrho_{+}}}+v_{x-}-v_{x+}, 0\right\}, \\
\frac{c_{+}}{\varrho_{+}}=\sqrt{\gamma \frac{p_{+}}{\varrho_{+}}}+\frac{\gamma+1}{2} \max \left\{\frac{p_{-}-p_{+}}{c_{-}}+v_{x-}-v_{x+}, 0\right\},
\end{array}\right. \\
& \text { if } p_{+} \leq p_{-},\left\{\begin{array}{l}
\frac{c_{+}}{\varrho_{+}}=\sqrt{\gamma \frac{p_{+}}{\varrho_{+}}}+\frac{\gamma+1}{2} \max \left\{\frac{p_{-}-p_{+}}{\varrho_{-} \sqrt{\gamma p_{-} / \varrho_{-}}}+v_{x-}-v_{x+}, 0\right\}, \\
\frac{c_{-}}{\varrho_{-}}=\sqrt{\gamma \frac{p_{-}}{\varrho_{-}}}+\frac{\gamma+1}{2} \max \left\{\frac{p_{+}-p_{-}}{c_{+}}+v_{x-}-v_{x+}, 0\right\} .
\end{array}\right.
\end{aligned}
$$

Then, intermediate values are computed using $c_{ \pm}=\varrho_{ \pm} \frac{c_{ \pm}}{\varrho_{ \pm}}$as

$$
\begin{array}{lll}
p_{-}^{*}=p_{+}^{*}=\frac{c_{+} p_{-}+c_{-} p_{+}-c_{-} c_{+}\left(v_{x+}-v_{x-}\right)}{c_{-}+c_{+}}, & \frac{1}{\varrho_{+}^{*}}=\frac{1}{\varrho_{+}}+\frac{c_{-}\left(v_{x+}-v_{x-}\right)+p_{+}-p_{-}}{c_{+}\left(c_{-}+c_{+}\right)}, \\
v_{x-}^{*}=v_{x+}^{*}=\frac{c_{-} v_{x-}+c_{+} v_{x+}+p_{-}-p_{+}}{c_{-}+c_{+}}, & \varepsilon_{-}^{*}=\varepsilon_{-}+\frac{\left(p_{-}^{*}\right)^{2}-p_{-}^{2}}{2 c_{-}^{2}}, \\
\frac{1}{\varrho_{-}^{*}}=\frac{1}{\varrho_{-}}+\frac{c_{+}\left(v_{x+}-v_{x-}\right)+p_{-}-p_{+}}{c_{-}\left(c_{-}+c_{+}\right)}, & \varepsilon_{+}^{*}=\varepsilon_{+}+\frac{\left(p_{+}^{*}\right)^{2}-p_{+}^{2}}{2 c_{+}^{2}} .
\end{array}
$$


Finally, the numerical fluxes are given by ( $f^{\text {num,y } y}$ analogously $)$

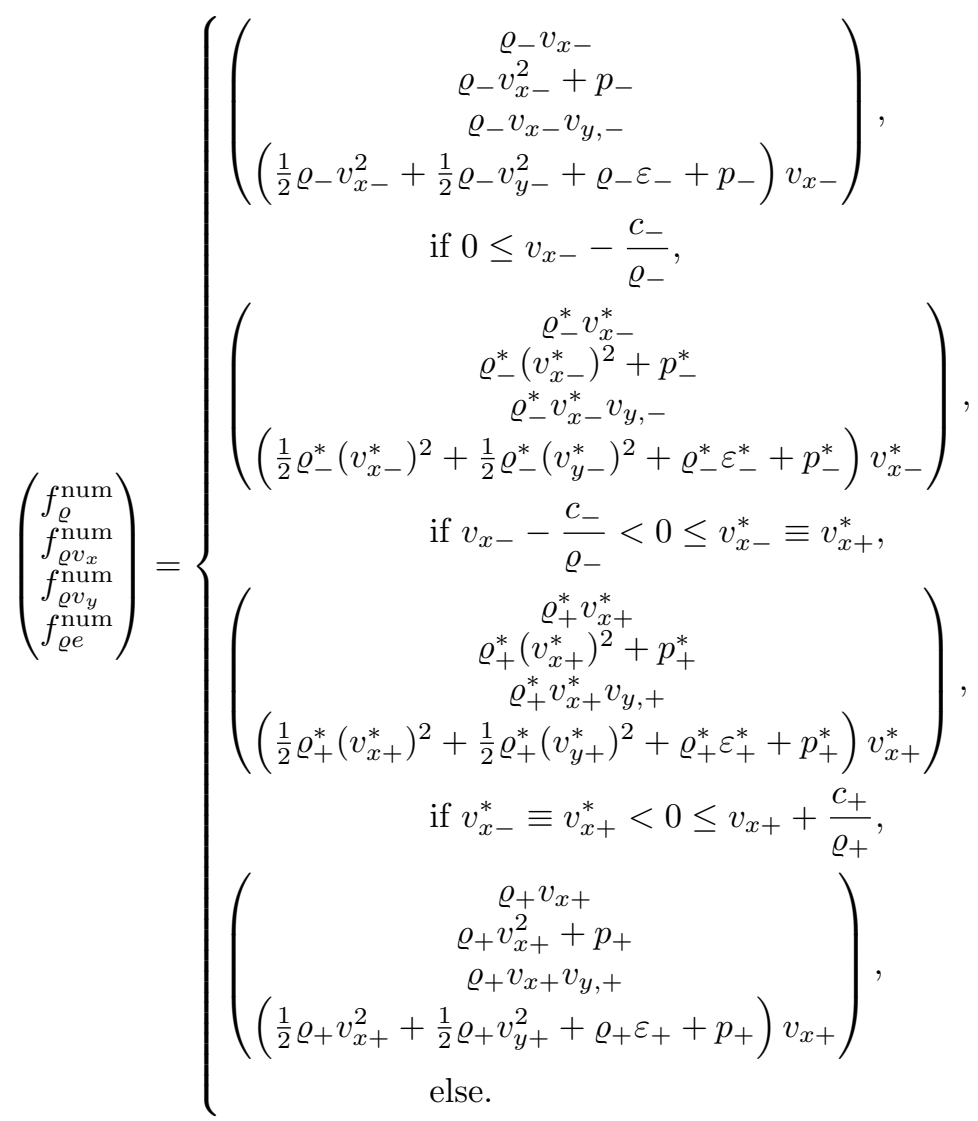

This flux is entropy stable and positivity preserving for $\varrho$ and $p$, with corresponding CFL condition

$$
\frac{\Delta t}{\Delta x} \max \left\{\left|v_{x-}-\frac{c_{-}}{\varrho_{-}}\right|,\left|v_{x+}+\frac{c_{+}}{\varrho_{+}}\right|\right\} \leq \frac{1}{2} .
$$

Additionally, it satisfies the maximum principle on the specific entropy $s$ and resolves stationary contact discontinuities with $v_{x} \equiv 0, p \equiv$ const exactly.

\section{Numerical Tests}

In this section, some numerical experiments using the methods described in the previous sections will be conducted. Unless stated otherwise, the ratio of specific heats is set to $\gamma=1.4$ and the three stage, third-order, strong stability preserving Runge-Kutta method of Gottlieb and Shu [13] will be used as time integration method. If a one-dimensional Riemann problem is considered, the exact solution is computed as described in [27, Section 4].

\subsection{Isentropic Vortex}

At first, the isentropic vortex problem of Shu [23, Problem 8 in section 5.1] will be used to test the methods for a smooth solution. The initial condition is given by

$$
\left(\begin{array}{c}
\varrho_{0} \\
\varrho v_{x, 0} \\
\varrho v_{y, 0} \\
\varrho e_{0}
\end{array}\right)=\left(\begin{array}{c}
\varrho_{\infty}\left(\frac{R T_{0}}{R T_{\infty}}\right)^{1 /(\gamma-1)} \\
\varrho_{0} v_{x, 0} \\
\varrho_{0} v_{y, 0} \\
\varrho \frac{v_{x, 0}^{2}+v_{y, 0}^{2}}{2}+\frac{p_{0}}{\gamma-1}
\end{array}\right),
$$

where

$$
v_{x, 0}=v_{x, \infty}+\delta v_{x}, \quad v_{y, 0}=v_{y, \infty}+\delta v_{y}, \quad p_{0}=\varrho_{0} R T_{0}, \quad R T_{0}=R T_{\infty}+\delta R T,
$$


and

$$
\begin{gathered}
\delta v_{x}(x, y)=-y \frac{\beta}{2 \pi} \exp \left(\frac{1-x^{2}-y^{2}}{2 r}\right), \quad \delta v_{y}(x, y)=x \frac{\beta}{2 \pi} \exp \left(\frac{1-x^{2}-y^{2}}{2 r}\right), \\
\delta R T(x, y)=-\frac{\gamma-1}{\gamma} \frac{\beta}{8 \pi^{2}} \exp \left(\frac{1-x^{2}-y^{2}}{r}\right) .
\end{gathered}
$$

The parameters have been chosen as

$$
\varrho_{\infty}=1, \quad v_{x, \infty}=1, \quad v_{y, \infty}=0, \quad R T_{\infty}=1, \quad \beta=5, \quad r=\frac{1}{4} .
$$

The solution is computed on the domain $[-5,5]^{2}$ during the time interval $[0,10]$. Thus, the perturbation is of the order of magnitude $\exp \left(\frac{1-5^{2}}{2 r}\right) \approx 10^{-21}$ at the boundary and should be approximately negligible for 64 bit floating point values. Therefore, the isentropic vortex should be advected with the free stream velocity and reach its initial position at $t=10$.

The Suliciu relaxation solver has been used as numerical flux and several volume fluxes have been used for the subcell flux differencing form:

- Central: The central flux $\{\{f\}\}$ resulting in a standard nodal DG method as described by Gassner, Winters, and Kopriva [12].

- Morinishi: The flux resulting in the split form of Morinishi [18] as described by Gassner, Winters, and Kopriva [12].

- Ducros: The flux resulting in the split form of Ducros, Laporte, Souleres, Guinot, Moinat, and Caruelle [8] as described by Gassner, Winters, and Kopriva [12].

- KG: The flux resulting in the split form of Kennedy and Gruber [17] as described by Gassner, Winters, and Kopriva [12].

- Pirozzoli: The flux resulting in the split form of Pirozzoli [19] as described by Gassner, Winters, and Kopriva [12].

- IR: The entropy conservative flux (45) of Roe [22] and Ismail and Roe [15].

- Ch: The entropy conservative flux (52) of Chandrashekar [3].

- $\varrho, v, \beta(2)$ : The flux (55).

- $\varrho, v, T(1)$ : The flux (75).

- $\varrho, v, \frac{1}{p}$ : The flux (61).

- $\varrho, v, T(2)$ : The flux (79).

- $\varrho, v, p$ : The flux (70).

- $\varrho, v, T$ (rev): The flux (106).

The errors (computed via the mass matrix $\underline{\underline{M}}$, i.e. Lobatto quadrature) in the density for varying polynomial degrees $p$ on a mesh using $10 \times 10$ elements are shown in Table 1 . As can be seen there, there is not much variance in the error for $p=1$. For $p \in\{2,3\}$, there are variations up to approximately $15 \%$ [e.g. $p=2$, Ducros, Morinishi and $p=3$, Ducros, $\varrho, v, T$ (rev)]. There does not seem to be any advantage of the entropy stable formulations compared to the other ones in this test case, similar to the results of Gassner, Winters, and Kopriva [12] for the Taylor Green vortex.

\subsection{Sod's Shock Tube: Subcell Flux Differencing}

In this section, the classical shock tube of Sod [24] will be used to test the semidiscretisations (10) using the volume terms (11) and the surface terms (12). The initial condition is given in primitive variables by

$$
\varrho_{0}(x)=\left\{\begin{array}{lc}
1, & x<\frac{1}{2}, \\
0.125, & \text { else },
\end{array} \quad v_{0}(x)=0, \quad p_{0}(x)= \begin{cases}1, & x<\frac{1}{2}, \\
0.1, & \text { else },\end{cases}\right.
$$


Table 1: Errors for the isentropic vortex problem (122) using $10 \times 10$ elements of varying polynomial degrees $p$, several volume fluxes and the Suliciu relaxation solver as numerical flux.

\begin{tabular}{c|c|c|c|c|c|c|c}
\hline & $\begin{array}{c}\text { Central } \\
\left\|\operatorname{err}_{\varrho}\right\|_{M}\end{array}$ & $\begin{array}{c}\text { Morinishi } \\
\left\|\operatorname{err}_{\varrho}\right\|_{M}\end{array}$ & $\begin{array}{c}\text { Ducros } \\
\left\|\operatorname{err}_{\varrho}\right\|_{M}\end{array}$ & $\begin{array}{c}\text { KG } \\
\left\|\operatorname{err}_{\varrho}\right\|_{M}\end{array}$ & $\begin{array}{c}\text { Pirozzoli } \\
\left\|\operatorname{err}_{\varrho}\right\|_{M}\end{array}$ & $\begin{array}{c}\text { IR } \\
\left\|\operatorname{err}_{\varrho}\right\|_{M}\end{array}$ & $\begin{array}{c}\text { Ch } \\
\left\|\operatorname{err}_{\varrho}\right\|_{M}\end{array}$ \\
\hline 1 & $1.20 \mathrm{e}-1$ & $1.21 \mathrm{e}-1$ & $1.20 \mathrm{e}-1$ & $1.20 \mathrm{e}-1$ & $1.20 \mathrm{e}-1$ & $1.20 \mathrm{e}-1$ & $1.21 \mathrm{e}-1$ \\
2 & $4.28 \mathrm{e}-2$ & $4.36 \mathrm{e}-2$ & $3.79 \mathrm{e}-2$ & $3.83 \mathrm{e}-2$ & $3.83 \mathrm{e}-2$ & $3.93 \mathrm{e}-2$ & $4.11 \mathrm{e}-2$ \\
3 & $1.73 \mathrm{e}-2$ & $1.81 \mathrm{e}-2$ & $1.60 \mathrm{e}-2$ & $1.62 \mathrm{e}-2$ & $1.62 \mathrm{e}-2$ & $1.64 \mathrm{e}-2$ & $1.80 \mathrm{e}-2$ \\
\hline \hline & $\varrho, v, \beta(2)$ & $\varrho, v, \frac{1}{p}$ & $\varrho, v, p$ & $\varrho, v, T(1)$ & $\varrho, v, T(2)$ & $\varrho, V, T\left(\mathrm{rev}^{2}\right)$ & \\
\hline & $\left\|\operatorname{err}_{\varrho}\right\|_{M}$ & $\left\|\mathrm{err}_{\varrho}\right\|_{M}$ & $\left\|\operatorname{err}_{\varrho}\right\|_{M}$ & $\left\|\operatorname{err}_{\varrho}\right\|_{M}$ & $\left\|\operatorname{err}_{\varrho}\right\|_{M}$ & $\left\|\operatorname{err}_{\varrho}\right\|_{M}$ & \\
\hline 1 & $1.21 \mathrm{e}-1$ & $1.21 \mathrm{e}-1$ & $1.21 \mathrm{e}-1$ & $1.21 \mathrm{e}-1$ & $1.20 \mathrm{e}-1$ & $1.20 \mathrm{e}-1$ & \\
2 & $4.13 \mathrm{e}-2$ & $4.11 \mathrm{e}-2$ & $4.11 \mathrm{e}-2$ & $4.11 \mathrm{e}-2$ & $3.93 \mathrm{e}-2$ & $4.12 \mathrm{e}-2$ & \\
3 & $1.81 \mathrm{e}-2$ & $1.79 \mathrm{e}-2$ & $1.79 \mathrm{e}-2$ & $1.80 \mathrm{e}-2$ & $1.64 \mathrm{e}-2$ & $1.89 \mathrm{e}-2$ & \\
\hline
\end{tabular}

and the conservative variables are computed via $\varrho v_{0}=\varrho_{0} v_{0}$ and $\varrho e_{0}=\frac{1}{2} \varrho_{0} v_{0}^{2}+\frac{p_{0}}{\gamma-1}$. The solution is computed on the domain $[-0.5,1.5]$ from $t=0$ until $t=0.25$ using 3000 time steps.

The error of the numerical solution for the density $\varrho$ is calculated using Lobatto quadrature, i.e. the diagonal mass matrix $\underline{M}$ of the SBP operator, for varying numbers of elements $N$ and polynomial degrees $p$. The results using the Suliciu relaxation solver and the local Lax-Friedrichs flux as numerical flux are shown in Tables 2 and 3, respectively.

There is some variance across the results for different volume fluxes up to approximately $25 \%$ [e.g. for $p=1, N=10,(\varrho, v, p)$ vs. $\varrho, v, T(\mathrm{rev})]$. However, there is no clear bias towards one volume flux across all combinations of the polynomial degree $p$ and the number of elements $N$ [e.g. for $p=1$ and $N=320,(\varrho, v, p)$ has a smaller error than $\varrho, v, T($ rev $)]$.

Contrary, comparing the numerical surface fluxes, there is some clear bias. Although the local Lax-Friedrichs flux yields a smaller error in some cases [e.g. $p=1, N=10,(\varrho, v, p)$ ], the Suliciu relaxation solver results in smaller errors if the resolution is good enough. Therefore, it can be considered superior to the LLF flux in this test case.

Additionally, the volume fluxes recovering the central form as well as the split forms of Morinishi [18], Ducros, Laporte, Souleres, Guinot, Moinat, and Caruelle [8], Kennedy and Gruber [17], and Pirozzoli [19] have been used. The relevant results are shown in the Tables 4 (Suliciu) and 5 (LLF). As can be seen there, the central flux and the splitting of Morinishi [18] are unstable. The splitting of Ducros, Laporte, Souleres, Guinot, Moinat, and Caruelle [8] crashes for polynomial degree $p=5$ whereas the other splittings remain stable. There is not much variance in the error for the stable calculations.

As for the entropy conservative volume fluxes, the Suliciu relaxation solver yields less error if the resolution is good enough. 


\begin{tabular}{|c|c|c|c|c|c|}
\hline 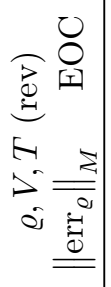 & 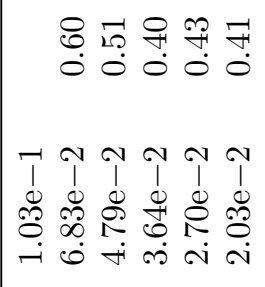 & 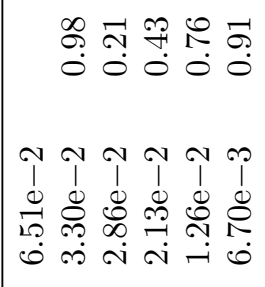 & 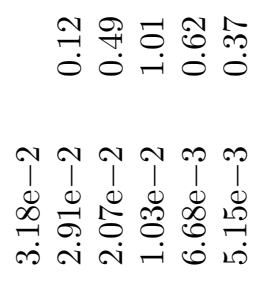 & 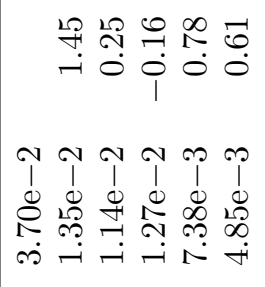 & 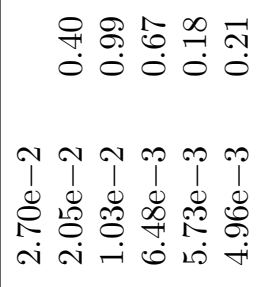 \\
\hline 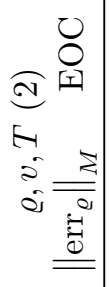 & 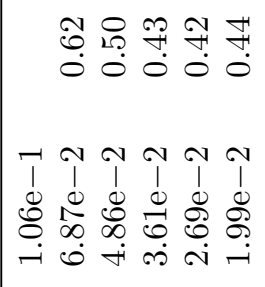 & 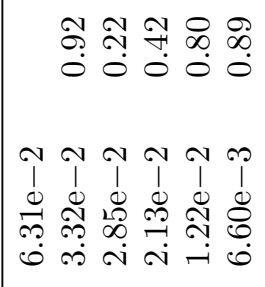 & 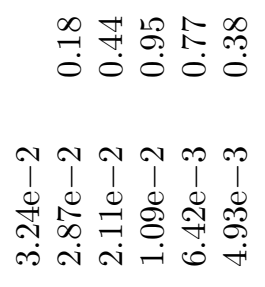 & 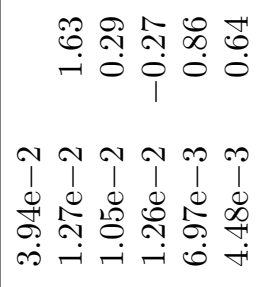 & 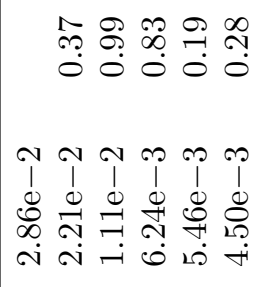 \\
\hline 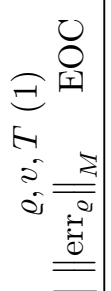 & 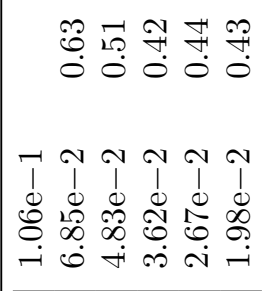 & 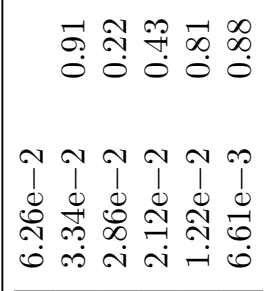 & 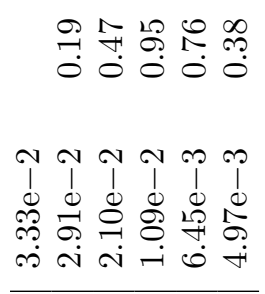 & 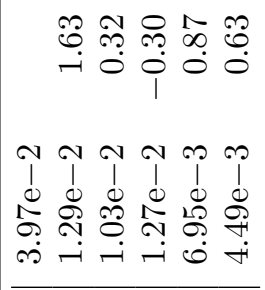 & 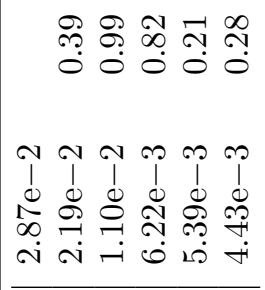 \\
\hline 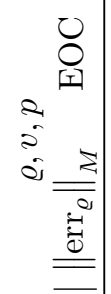 & 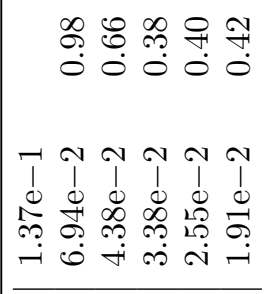 & 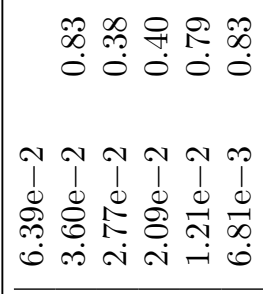 & 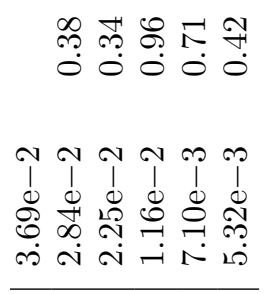 & 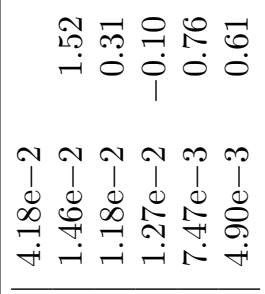 & 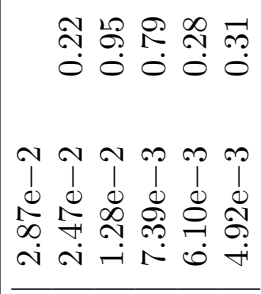 \\
\hline 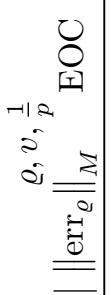 & 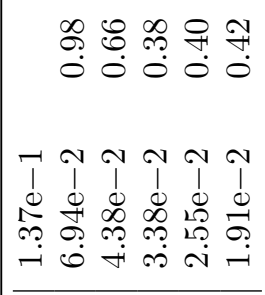 & 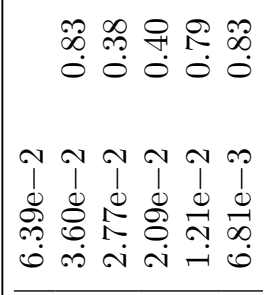 & 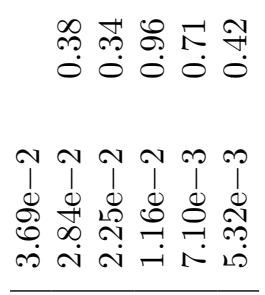 & 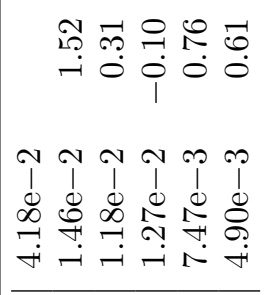 & 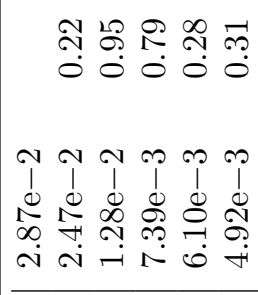 \\
\hline 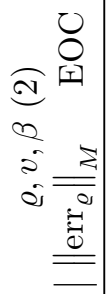 & 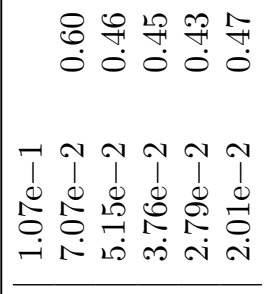 & 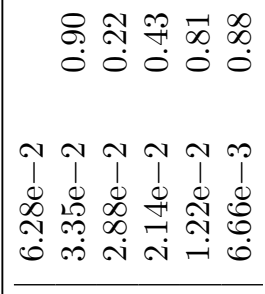 & 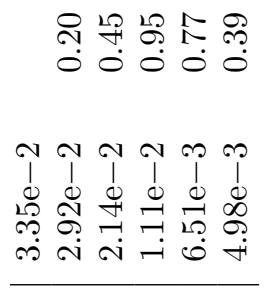 & 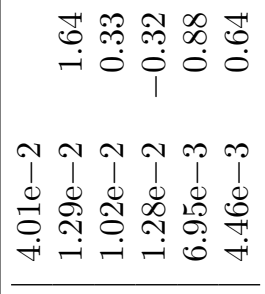 & 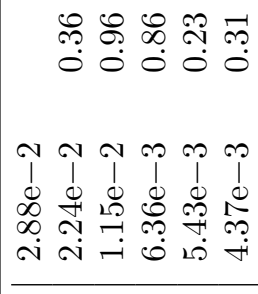 \\
\hline 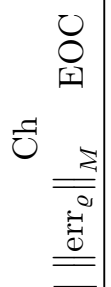 & 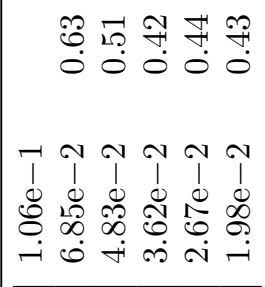 & 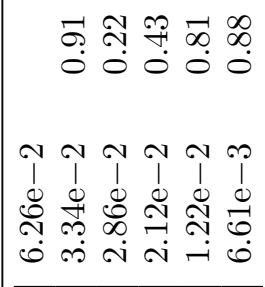 & 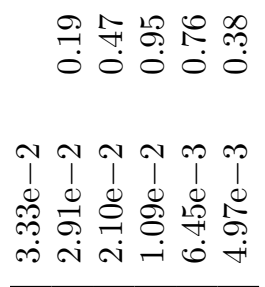 & 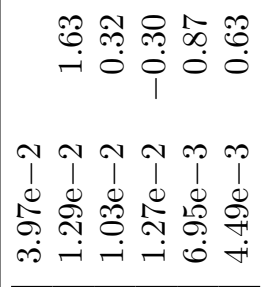 & 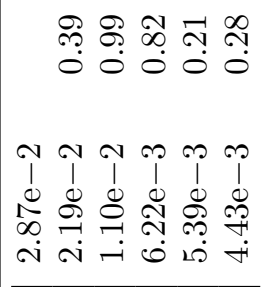 \\
\hline 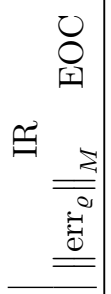 & 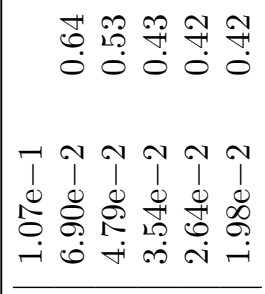 & 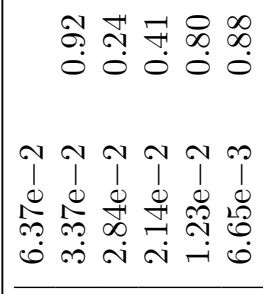 & 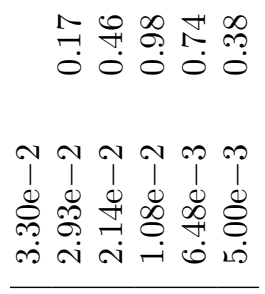 & 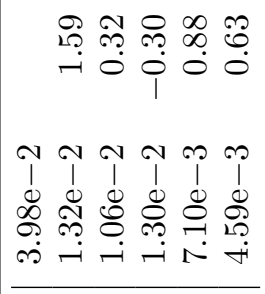 & 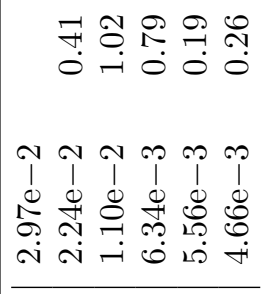 \\
\hline$z$ & 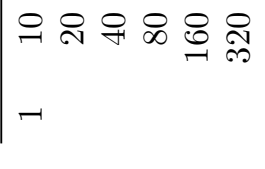 & 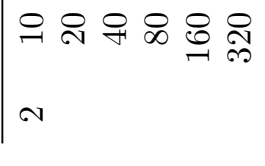 & 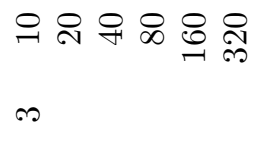 & 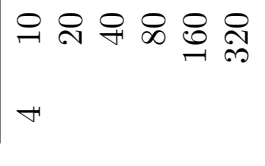 & 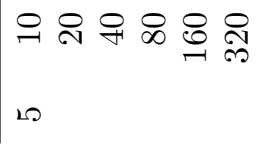 \\
\hline
\end{tabular}




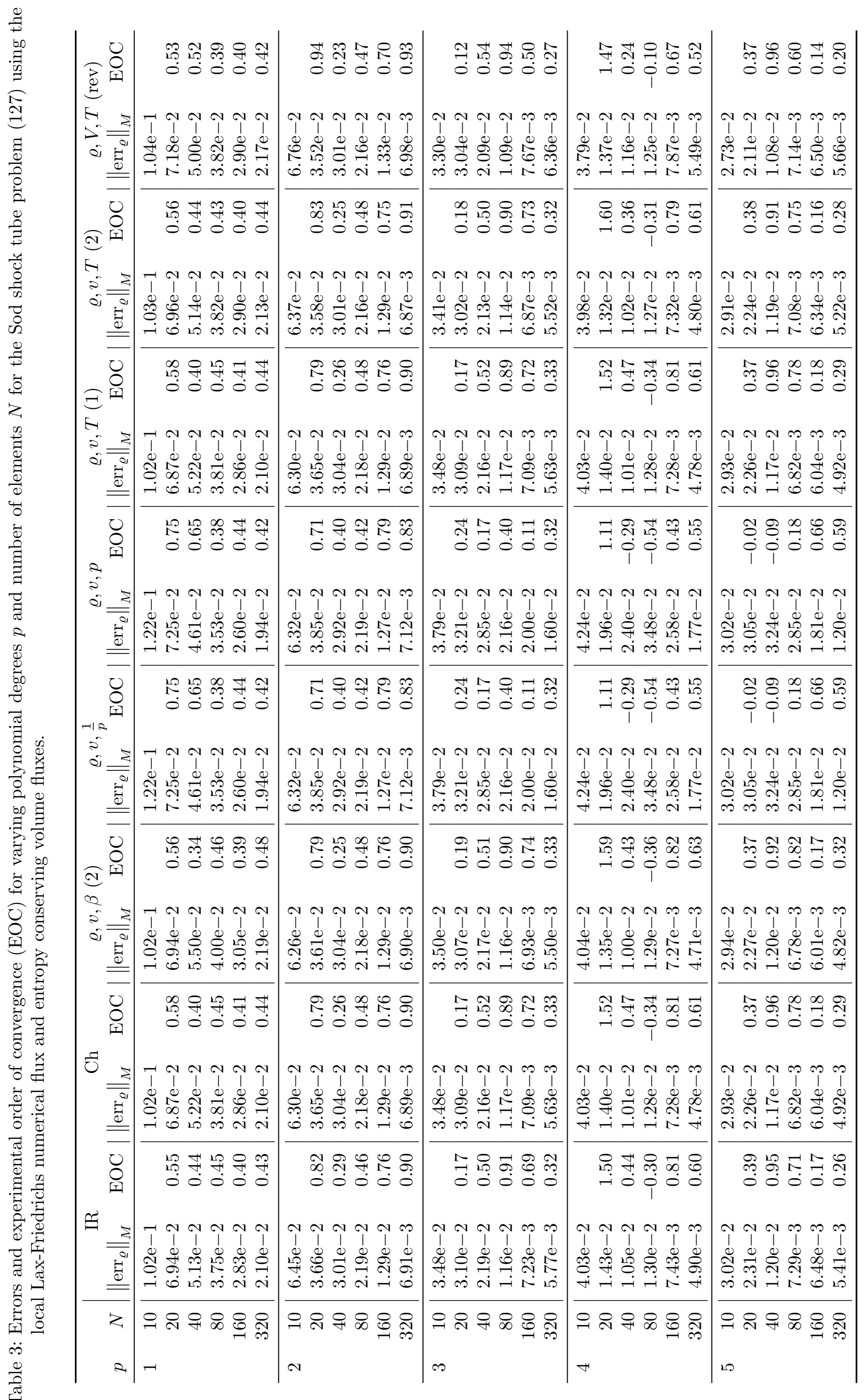


Table 4: Errors and experimental order of convergence (EOC) for varying polynomial degrees $p$ and number of elements $N$ for the Sod shock tube problem (127) using the Suliciu relaxation solver as numerical flux and "simple" volume fluxes.

\begin{tabular}{|c|c|c|c|c|c|c|c|c|c|c|c|c|}
\hline \multirow[b]{2}{*}{$p$} & \multirow[b]{2}{*}{$N$} & \multicolumn{3}{|c|}{ Central } & \multicolumn{2}{|c|}{ Morinishi } & \multicolumn{2}{|c|}{ Ducros } & \multicolumn{2}{|c|}{$\mathrm{KG}$} & \multicolumn{2}{|c|}{ Pirozzoli } \\
\hline & & || $\operatorname{err}_{\varrho} \mid$ & $M$ & EOC & $\mid \operatorname{err}_{\varrho} \|_{M}$ & $\mathrm{EOC}$ & $\left\|\operatorname{err}_{\varrho}\right\|_{M}$ & EOC & $\left\|\operatorname{err}_{\varrho}\right\|_{M}$ & EOC & $\left\|\operatorname{err}_{\varrho}\right\|_{M}$ & $\mathrm{EOC}$ \\
\hline 1 & 10 & & * & & $*$ & & $1.04 \mathrm{e}-1$ & & $1.05 \mathrm{e}-1$ & & $1.06 \mathrm{e}-1$ & \\
\hline & 20 & & $*$ & $*$ & $*$ & $*$ & $6.66 \mathrm{e}-2$ & 0.65 & $6.64 \mathrm{e}-2$ & 0.66 & $6.68 \mathrm{e}-2$ & 0.66 \\
\hline & 40 & & * & $*$ & $*$ & $*$ & $4.20 \mathrm{e}-2$ & 0.67 & $4.51 \mathrm{e}-2$ & 0.56 & $4.59 \mathrm{e}-2$ & 0.54 \\
\hline & 80 & & * & $*$ & $*$ & $*$ & $3.26 \mathrm{e}-2$ & 0.36 & $3.48 \mathrm{e}-2$ & 0.37 & $3.50 \mathrm{e}-2$ & 0.39 \\
\hline & 160 & & * & $*$ & $*$ & $*$ & $2.45 \mathrm{e}-2$ & 0.41 & $2.59 \mathrm{e}-2$ & 0.43 & $2.58 \mathrm{e}-2$ & 0.44 \\
\hline & 320 & & * & $*$ & $*$ & $*$ & $1.86 \mathrm{e}-2$ & 0.40 & $1.95 \mathrm{e}-2$ & 0.41 & $1.95 \mathrm{e}-2$ & 0.40 \\
\hline 2 & 10 & & $*$ & & $8.74 \mathrm{e}-2$ & & $6.38 \mathrm{e}-2$ & & $6.40 \mathrm{e}-2$ & & $6.39 \mathrm{e}-2$ & \\
\hline & 20 & & * & $*$ & $*$ & $*$ & $3.36 \mathrm{e}-2$ & 0.93 & $3.41 \mathrm{e}-2$ & 0.91 & $3.41 \mathrm{e}-2$ & 0.91 \\
\hline & 40 & & * & $*$ & $*$ & $*$ & $2.56 \mathrm{e}-2$ & 0.39 & $2.66 \mathrm{e}-2$ & 0.36 & $2.69 \mathrm{e}-2$ & 0.34 \\
\hline & 80 & & * & $*$ & * & * & $1.93 \mathrm{e}-2$ & 0.41 & $2.00 \mathrm{e}-2$ & 0.41 & $2.00 \mathrm{e}-2$ & 0.43 \\
\hline & 160 & & $*$ & $*$ & $*$ & $*$ & $1.18 \mathrm{e}-2$ & 0.71 & $1.22 \mathrm{e}-2$ & 0.71 & $1.24 \mathrm{e}-2$ & 0.69 \\
\hline & 320 & & * & * & $*$ & * & $6.32 \mathrm{e}-3$ & 0.90 & $6.47 \mathrm{e}-3$ & 0.91 & $6.55 \mathrm{e}-3$ & 0.92 \\
\hline 3 & 10 & & $*$ & & $*$ & & $3.29 \mathrm{e}-2$ & & $3.30 \mathrm{e}-2$ & & $3.33 \mathrm{e}-2$ & \\
\hline & 20 & & * & * & * & $*$ & $2.51 \mathrm{e}-2$ & 0.39 & $2.56 \mathrm{e}-2$ & 0.36 & $2.60 \mathrm{e}-2$ & 0.36 \\
\hline & 40 & & $*$ & $*$ & $*$ & $*$ & $1.74 \mathrm{e}-2$ & 0.52 & $1.93 \mathrm{e}-2$ & 0.41 & $1.93 \mathrm{e}-2$ & 0.43 \\
\hline & 80 & & * & $*$ & $*$ & $*$ & $9.93 \mathrm{e}-3$ & 0.81 & $9.89 \mathrm{e}-3$ & 0.97 & $9.79 \mathrm{e}-3$ & 0.98 \\
\hline & 160 & & * & * & $*$ & $*$ & $6.05 \mathrm{e}-3$ & 0.72 & $5.97 \mathrm{e}-3$ & 0.73 & $6.04 \mathrm{e}-3$ & 0.70 \\
\hline & 320 & & * & * & * & $*$ & $5.03 \mathrm{e}-3$ & 0.27 & $4.83 \mathrm{e}-3$ & 0.31 & $4.85 \mathrm{e}-3$ & 0.32 \\
\hline 4 & 10 & & $*$ & & $*$ & & $3.48 \mathrm{e}-2$ & & $3.62 \mathrm{e}-2$ & & $3.63 \mathrm{e}-2$ & \\
\hline & 20 & & * & $*$ & $*$ & $*$ & $1.35 \mathrm{e}-2$ & 1.37 & $1.26 \mathrm{e}-2$ & 1.52 & $1.27 \mathrm{e}-2$ & 1.51 \\
\hline & 40 & & $*$ & $*$ & $*$ & $*$ & $1.21 \mathrm{e}-2$ & 0.15 & $1.17 \mathrm{e}-2$ & 0.11 & $1.17 \mathrm{e}-2$ & 0.12 \\
\hline & 80 & & * & $*$ & $*$ & $*$ & $1.03 \mathrm{e}-2$ & 0.23 & $1.13 \mathrm{e}-2$ & 0.05 & $1.15 \mathrm{e}-2$ & 0.03 \\
\hline & 160 & & $*$ & $*$ & * & $*$ & $7.13 \mathrm{e}-3$ & 0.53 & $7.04 \mathrm{e}-3$ & 0.68 & $7.15 \mathrm{e}-3$ & 0.68 \\
\hline & 320 & & $*$ & $*$ & $*$ & $*$ & $5.17 \mathrm{e}-3$ & 0.46 & $4.77 \mathrm{e}-3$ & 0.56 & $4.74 \mathrm{e}-3$ & 0.59 \\
\hline 5 & 10 & & * & & $*$ & & $*$ & & $2.53 \mathrm{e}-2$ & & $2.59 \mathrm{e}-2$ & \\
\hline & 20 & & $*$ & $*$ & $*$ & $*$ & $*$ & * & $1.92 \mathrm{e}-2$ & 0.40 & $1.92 \mathrm{e}-2$ & 0.43 \\
\hline & 40 & & $*$ & $*$ & $*$ & $*$ & $*$ & $*$ & $9.55 \mathrm{e}-3$ & 1.00 & $9.40 \mathrm{e}-3$ & 1.03 \\
\hline & 80 & & * & $*$ & $*$ & $*$ & $*$ & $*$ & $6.47 \mathrm{e}-3$ & 0.56 & $6.47 \mathrm{e}-3$ & 0.54 \\
\hline & 160 & & $*$ & $*$ & $*$ & $*$ & $*$ & $*$ & $5.68 \mathrm{e}-3$ & 0.19 & $5.68 \mathrm{e}-3$ & 0.19 \\
\hline & 320 & & $*$ & $*$ & $*$ & $*$ & $*$ & $*$ & $5.24 \mathrm{e}-3$ & 0.12 & $5.19 \mathrm{e}-3$ & 0.13 \\
\hline
\end{tabular}


Table 5: Errors and experimental order of convergence (EOC) for varying polynomial degrees $p$ and number of elements $N$ for the Sod shock tube problem (127) using the local Lax-Friedrichs numerical flux and "simple" volume fluxes.

\begin{tabular}{|c|c|c|c|c|c|c|c|c|c|c|c|c|}
\hline \multirow[b]{2}{*}{$p$} & \multirow[b]{2}{*}{$N$} & \multicolumn{3}{|c|}{ Central } & \multicolumn{2}{|c|}{ Morinishi } & \multicolumn{2}{|c|}{ Ducros } & \multicolumn{2}{|c|}{$\mathrm{KG}$} & \multicolumn{2}{|c|}{ Pirozzoli } \\
\hline & & $\left\|\operatorname{err}_{\varrho}\right\|_{\Lambda}$ & 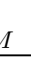 & EOC & $\left\|\operatorname{err}_{\varrho}\right\|_{M}$ & $\mathrm{EOC}$ & $\left\|\operatorname{err}_{\varrho}\right\|_{M}$ & EOC & $\left\|\operatorname{err}_{\varrho}\right\|_{M}$ & EOC & $\left\|\operatorname{err}_{\varrho}\right\|_{M}$ & EOC \\
\hline \multirow[t]{6}{*}{1} & 10 & & * & & $*$ & & $1.03 \mathrm{e}-1$ & & $1.02 \mathrm{e}-1$ & & $1.02 \mathrm{e}-1$ & \\
\hline & 20 & & * & * & * & * & $6.74 \mathrm{e}-2$ & 0.61 & $6.75 \mathrm{e}-2$ & 0.60 & $6.75 \mathrm{e}-2$ & 0.60 \\
\hline & 40 & & * & * & $*$ & * & $4.38 \mathrm{e}-2$ & 0.62 & $4.82 \mathrm{e}-2$ & 0.49 & $4.94 \mathrm{e}-2$ & 0.45 \\
\hline & 80 & & * & * & * & * & $3.38 \mathrm{e}-2$ & 0.37 & $3.63 \mathrm{e}-2$ & 0.41 & $3.68 \mathrm{e}-2$ & 0.42 \\
\hline & 160 & & * & $*$ & $*$ & $*$ & $2.60 \mathrm{e}-2$ & 0.38 & $2.76 \mathrm{e}-2$ & 0.39 & $2.77 \mathrm{e}-2$ & 0.41 \\
\hline & 320 & & * & * & * & * & * & * & $2.09 \mathrm{e}-2$ & 0.40 & $2.08 \mathrm{e}-2$ & 0.41 \\
\hline \multirow[t]{6}{*}{2} & 10 & $8.35 \mathrm{e}-$ & & & $5.87 \mathrm{e}-2$ & & $6.60 \mathrm{e}-2$ & & $6.54 \mathrm{e}-2$ & & $6.46 \mathrm{e}-2$ & \\
\hline & 20 & & * & * & * & * & $3.42 \mathrm{e}-2$ & 0.95 & $3.52 \mathrm{e}-2$ & 0.90 & $3.53 \mathrm{e}-2$ & 0.87 \\
\hline & 40 & & * & * & * & * & $2.67 \mathrm{e}-2$ & 0.36 & $2.78 \mathrm{e}-2$ & 0.34 & $2.82 \mathrm{e}-2$ & 0.33 \\
\hline & 80 & & * & * & * & * & $1.93 \mathrm{e}-2$ & 0.47 & $2.02 \mathrm{e}-2$ & 0.47 & $2.02 \mathrm{e}-2$ & 0.48 \\
\hline & 160 & & * & * & * & * & $1.24 \mathrm{e}-2$ & 0.65 & $1.27 \mathrm{e}-2$ & 0.66 & $1.29 \mathrm{e}-2$ & 0.65 \\
\hline & 320 & & * & $*$ & * & $*$ & $6.54 \mathrm{e}-3$ & 0.92 & $6.66 \mathrm{e}-3$ & 0.94 & $6.73 \mathrm{e}-3$ & 0.94 \\
\hline \multirow[t]{6}{*}{3} & 10 & & * & & $*$ & & $3.31 \mathrm{e}-2$ & & $3.44 \mathrm{e}-2$ & & $3.50 \mathrm{e}-2$ & \\
\hline & 20 & & * & * & * & $*$ & $2.58 \mathrm{e}-2$ & 0.36 & $2.72 \mathrm{e}-2$ & 0.34 & $2.78 \mathrm{e}-2$ & 0.34 \\
\hline & 40 & & * & $*$ & $*$ & * & $1.74 \mathrm{e}-2$ & 0.57 & $1.93 \mathrm{e}-2$ & 0.50 & $1.93 \mathrm{e}-2$ & 0.52 \\
\hline & 80 & & * & * & * & * & $1.05 \mathrm{e}-2$ & 0.74 & $1.04 \mathrm{e}-2$ & 0.88 & $1.04 \mathrm{e}-2$ & 0.89 \\
\hline & 160 & & * & * & * & * & $6.91 \mathrm{e}-3$ & 0.60 & $6.57 \mathrm{e}-3$ & 0.67 & $6.63 \mathrm{e}-3$ & 0.65 \\
\hline & 320 & & * & * & * & * & $6.08 \mathrm{e}-3$ & 0.19 & $5.58 \mathrm{e}-3$ & 0.24 & $5.59 \mathrm{e}-3$ & 0.25 \\
\hline \multirow[t]{6}{*}{4} & 10 & & * & & * & & $3.49 \mathrm{e}-2$ & & $3.68 \mathrm{e}-2$ & & $3.70 \mathrm{e}-2$ & \\
\hline & 20 & & * & * & * & * & $1.27 \mathrm{e}-2$ & 1.46 & $1.23 \mathrm{e}-2$ & 1.58 & $1.26 \mathrm{e}-2$ & 1.56 \\
\hline & 40 & & * & * & * & * & $1.18 \mathrm{e}-2$ & 0.11 & $1.13 \mathrm{e}-2$ & 0.13 & $1.14 \mathrm{e}-2$ & 0.15 \\
\hline & 80 & & * & * & * & * & $1.02 \mathrm{e}-2$ & 0.21 & $1.13 \mathrm{e}-2$ & 0.00 & $1.15 \mathrm{e}-2$ & -0.01 \\
\hline & 160 & & * & * & $*$ & * & $7.43 \mathrm{e}-3$ & 0.46 & $7.49 \mathrm{e}-3$ & 0.59 & $7.57 \mathrm{e}-3$ & 0.60 \\
\hline & 320 & & * & $*$ & $*$ & $*$ & $5.47 \mathrm{e}-3$ & 0.44 & $5.22 \mathrm{e}-3$ & 0.52 & $5.15 \mathrm{e}-3$ & 0.56 \\
\hline \multirow[t]{6}{*}{5} & 10 & & * & & $*$ & & * & & $2.57 \mathrm{e}-2$ & & $2.65 \mathrm{e}-2$ & \\
\hline & 20 & & * & $*$ & * & $*$ & * & $*$ & $1.93 \mathrm{e}-2$ & 0.41 & $1.93 \mathrm{e}-2$ & 0.46 \\
\hline & 40 & & * & * & * & * & $*$ & $*$ & $1.11 \mathrm{e}-2$ & 0.80 & $1.08 \mathrm{e}-2$ & 0.84 \\
\hline & 80 & & * & * & * & * & * & $*$ & $7.68 \mathrm{e}-3$ & 0.54 & $7.53 \mathrm{e}-3$ & 0.52 \\
\hline & 160 & & * & * & * & * & * & $*$ & $6.94 \mathrm{e}-3$ & 0.15 & $6.82 \mathrm{e}-3$ & 0.14 \\
\hline & 320 & & * & * & * & * & * & * & $6.35 \mathrm{e}-3$ & 0.13 & $6.18 \mathrm{e}-3$ & 0.14 \\
\hline
\end{tabular}




\subsection{Modified Version of Sod's Shock Tube: Subcell Flux Differencing}

In this section, the modified version of the shock tube of Sod [24] as described by Toro [27, Section 6.4, Test 1] will be used to test the semidiscretisations (10) using the volume terms (11) and the surface terms (12). The initial condition is given in primitive variables by

$$
\varrho_{0}(x)=\left\{\begin{array}{ll}
1, & x<\frac{3}{10}, \\
0.125 & \text { else },
\end{array}, \quad v_{0}(x)=\left\{\begin{array}{ll}
0.75, & x<\frac{3}{10}, \\
0 & \text { else },
\end{array} \quad p_{0}(x)= \begin{cases}1, & x<\frac{3}{10}, \\
0.1, & \text { else }\end{cases}\right.\right.
$$

and the conservative variables are again computed via $\varrho v_{0}=\varrho_{0} v_{0}$ and $\varrho e_{0}=\frac{1}{2} \varrho_{0} v_{0}^{2}+\frac{p_{0}}{\gamma-1}$. The solution is computed on the domain $[0,1]$ from $t=0$ until $t=0.2$ using 15000 steps and the error is computed as in section 7.2.

The results using the Suliciu relaxation solver and the local Lax-Friedrichs flux for varying polynomial degrees $p$ and number of elements $N$ are shown in Table 6 and 7, respectively. Again, there are some variances across the volume fluxes of up to $33 \%$ [e.g. $p=4, N=10,(\varrho, v, p)$ vs. $(\varrho, v, \beta(2))]$ but no flux seems to be clearly superior. As in the previous test case in section 7.2, the Suliciu solver performs better than the LLF flux - at least, if the resolution is good enough.

As in the previous section 7.2, the volume fluxes recovering the central form as well as the split forms of Morinishi [18], Ducros, Laporte, Souleres, Guinot, Moinat, and Caruelle [8], Kennedy and Gruber [17], and Pirozzoli [19] have been used. The relevant results are shown in the Tables 8 (Suliciu) and 9 (LLF).

Contrary to the results of the unmodified shock tube of Sod, all calculations are stable for low resolution. The splitting of Morinishi [18] blows up at first if the Suliciu solver is used, while the central flux crashes at first for the LLF flux. Moreover, even the splittings of Kennedy and Gruber [17] and Pirozzoli [19] that remained stable in the previous section 7.2 blow up for higher polynomial degrees. 


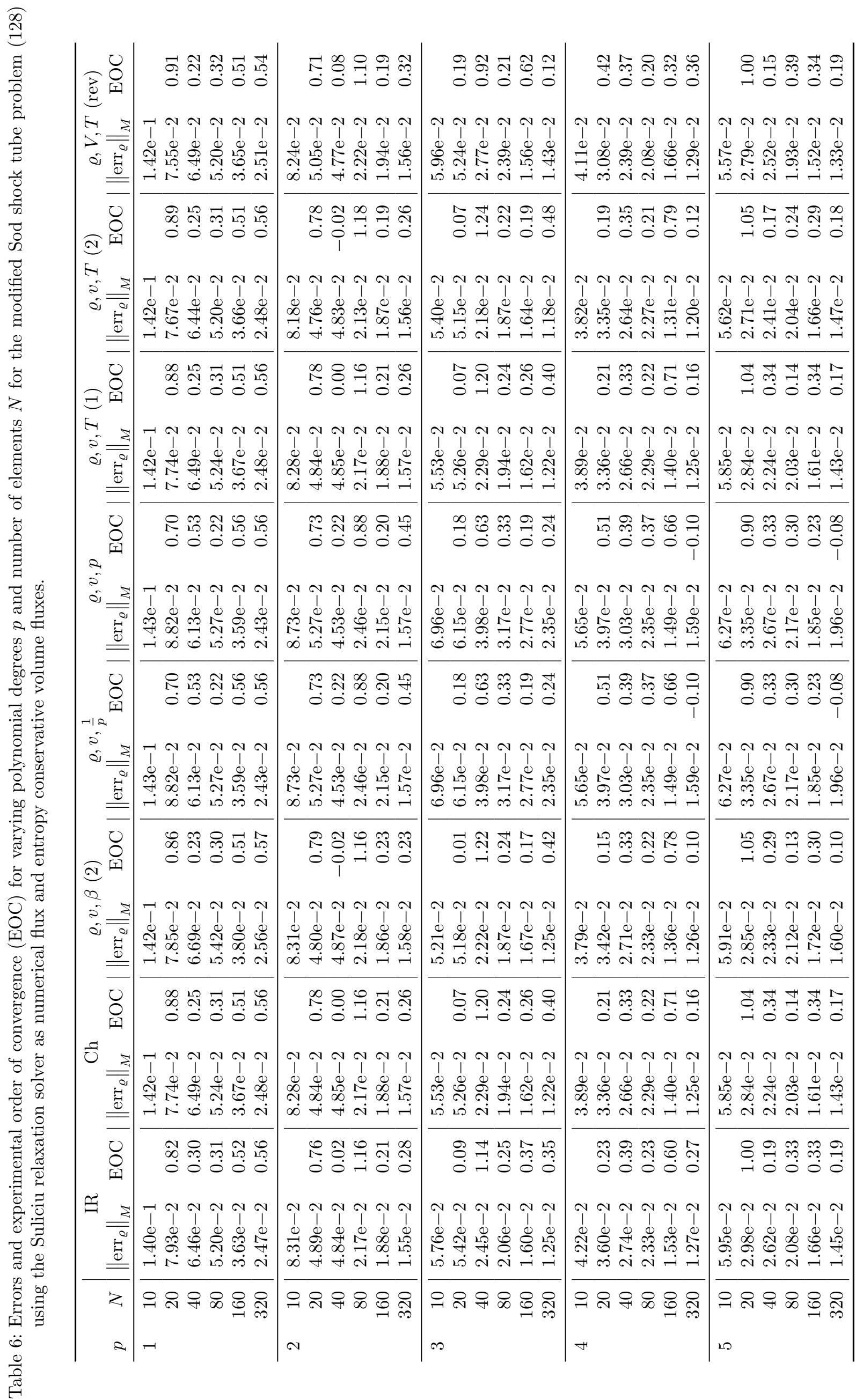




\begin{tabular}{|c|c|c|c|c|c|}
\hline 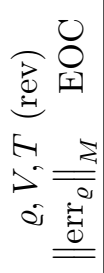 & 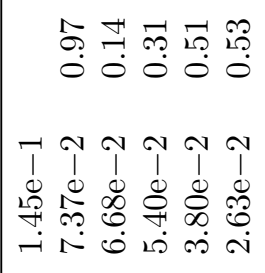 & 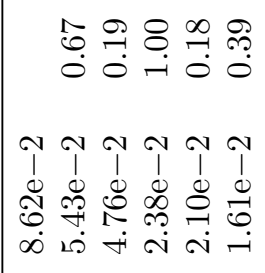 & 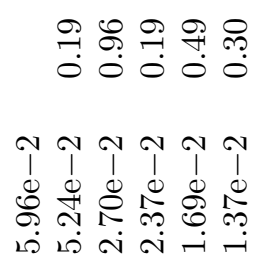 & 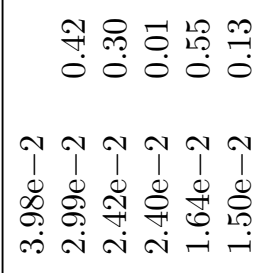 & 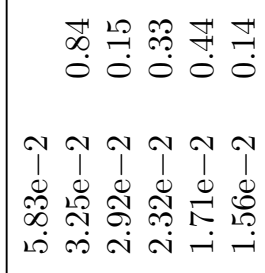 \\
\hline 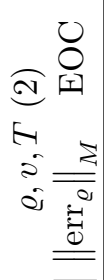 & 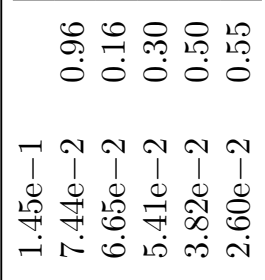 & 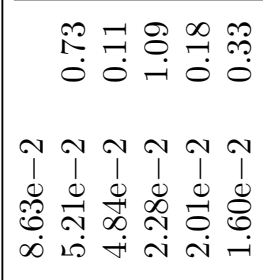 & 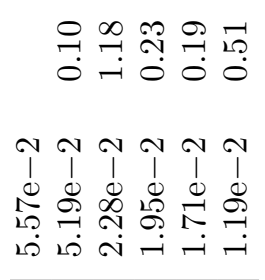 & 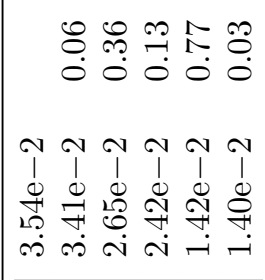 & 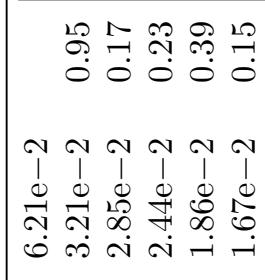 \\
\hline 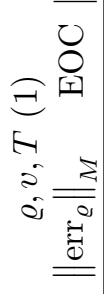 & 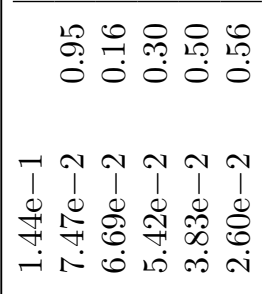 & 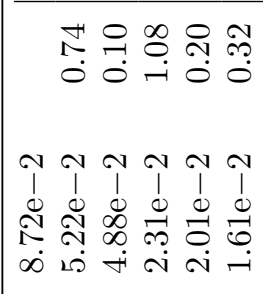 & 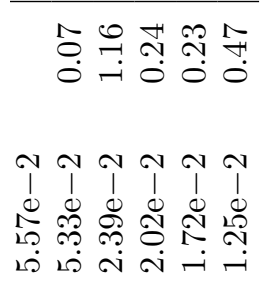 & 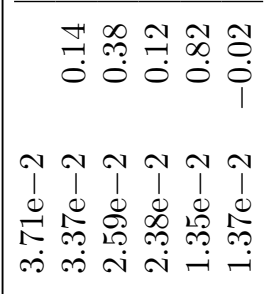 & 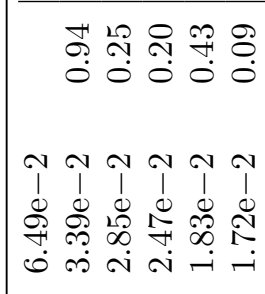 \\
\hline 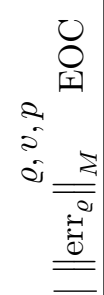 & 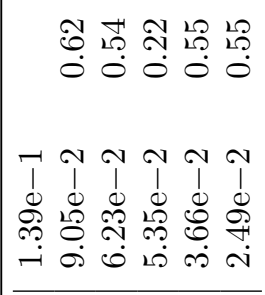 & 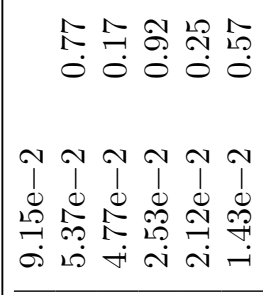 & 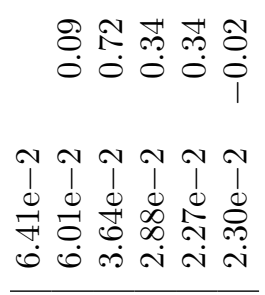 & 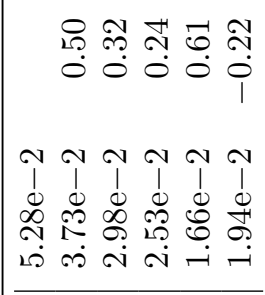 & 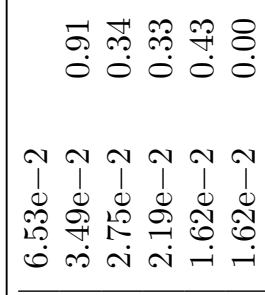 \\
\hline 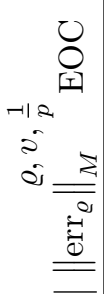 & 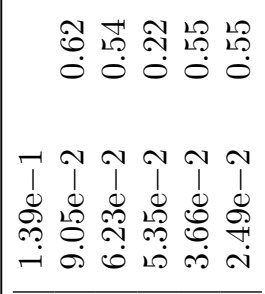 & 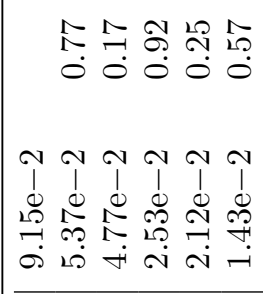 & 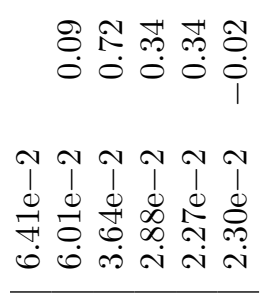 & 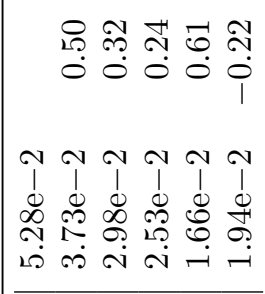 & 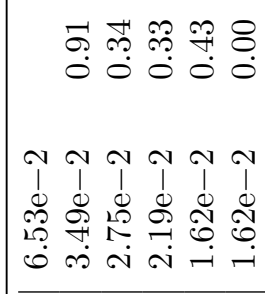 \\
\hline 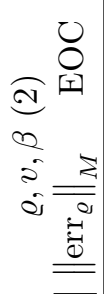 & 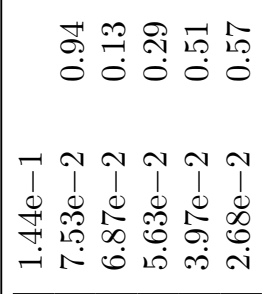 & 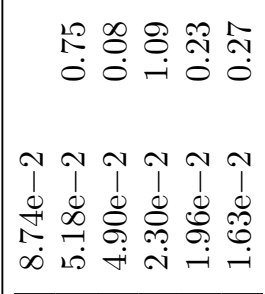 & 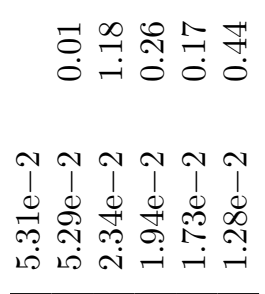 & 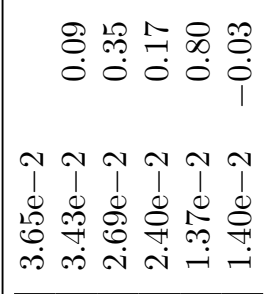 & 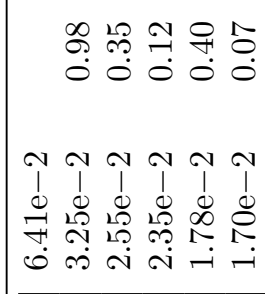 \\
\hline 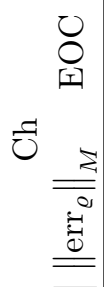 & 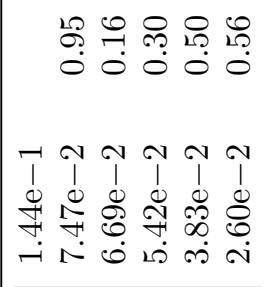 & 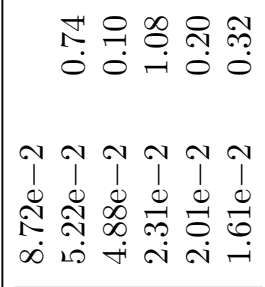 & 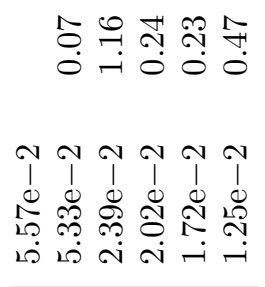 & 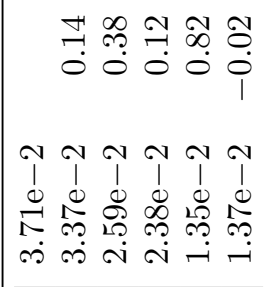 & 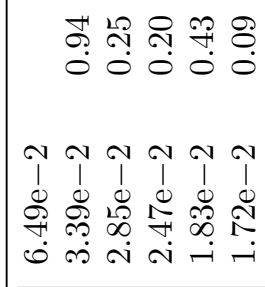 \\
\hline 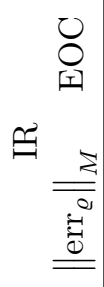 & 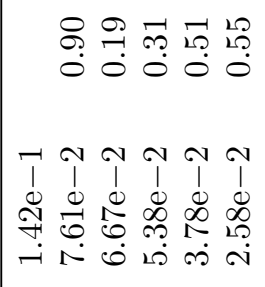 & 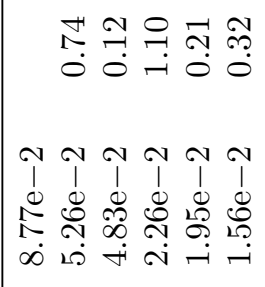 & 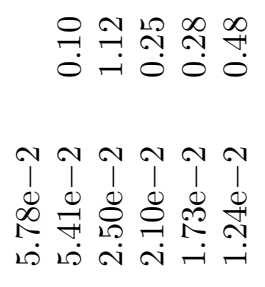 & 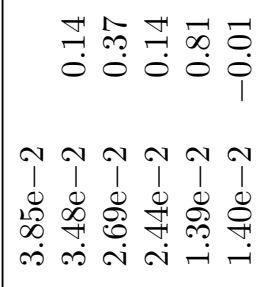 & 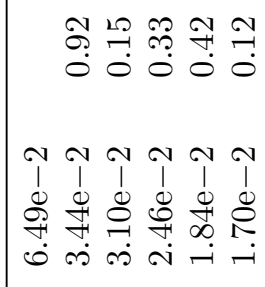 \\
\hline$z$ & 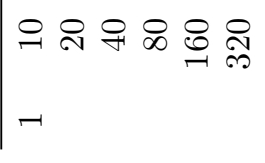 & 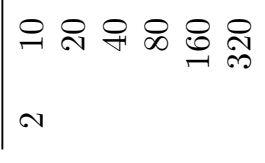 & 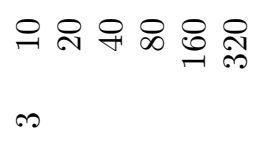 & 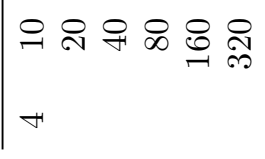 & $\underset{\sim}{ㅇ ㅠ ~}$ \\
\hline
\end{tabular}


Table 8: Errors and experimental order of convergence (EOC) for varying polynomial degrees $p$ and number of elements $N$ for the modified Sod shock tube problem (128) using the Suliciu relaxation solver as numerical flux and "simple" volume fluxes.

\begin{tabular}{|c|c|c|c|c|c|c|c|c|c|c|c|}
\hline \multirow[b]{2}{*}{$p$} & \multirow[b]{2}{*}{$N$} & \multicolumn{2}{|c|}{ Central } & \multicolumn{2}{|c|}{ Morinishi } & \multicolumn{2}{|c|}{ Ducros } & \multicolumn{2}{|c|}{$\mathrm{KG}$} & \multicolumn{2}{|c|}{ Pirozzoli } \\
\hline & & $\left\|\operatorname{err}_{\varrho}\right\|_{M}$ & $\mathrm{EOC}$ & $\left\|\operatorname{err}_{\varrho}\right\|_{M}$ & EOC & $\left\|\operatorname{err}_{\varrho}\right\|_{M}$ & $\mathrm{EOC}$ & $\left\|\operatorname{err}_{\varrho}\right\|_{M}$ & EOC & $\left\|\operatorname{err}_{\varrho}\right\|_{M}$ & EOC \\
\hline 1 & 10 & $1.42 \mathrm{e}-1$ & & $1.42 \mathrm{e}-1$ & & $1.34 \mathrm{e}-1$ & & $1.33 \mathrm{e}-1$ & & $1.33 \mathrm{e}-1$ & \\
\hline & 20 & $7.46 \mathrm{e}-2$ & 0.93 & $8.55 \mathrm{e}-2$ & 0.74 & $7.21 \mathrm{e}-2$ & 0.89 & $7.41 \mathrm{e}-2$ & 0.85 & $7.48 \mathrm{e}-2$ & 0.83 \\
\hline & 40 & $6.63 \mathrm{e}-2$ & 0.17 & $1.05 \mathrm{e}-1$ & -0.29 & $5.71 \mathrm{e}-2$ & 0.34 & $6.07 \mathrm{e}-2$ & 0.29 & $6.17 \mathrm{e}-2$ & 0.28 \\
\hline & 80 & $6.00 \mathrm{e}-2$ & 0.14 & $*$ & * & $4.49 \mathrm{e}-2$ & 0.35 & $4.79 \mathrm{e}-2$ & 0.34 & $4.86 \mathrm{e}-2$ & 0.34 \\
\hline & 160 & $5.25 \mathrm{e}-2$ & 0.19 & * & $*$ & $3.10 \mathrm{e}-2$ & 0.53 & $3.35 \mathrm{e}-2$ & 0.52 & $3.42 \mathrm{e}-2$ & 0.51 \\
\hline & 320 & $4.86 \mathrm{e}-2$ & 0.11 & $*$ & $*$ & $2.14 \mathrm{e}-2$ & 0.53 & $2.34 \mathrm{e}-2$ & 0.52 & $2.40 \mathrm{e}-2$ & 0.51 \\
\hline 2 & 10 & * & & $8.38 \mathrm{e}-2$ & & $7.83 \mathrm{e}-2$ & & $8.03 \mathrm{e}-2$ & & $8.02 \mathrm{e}-2$ & \\
\hline & 20 & * & * & $4.62 \mathrm{e}-2$ & 0.86 & $4.97 \mathrm{e}-2$ & 0.66 & $5.10 \mathrm{e}-2$ & 0.66 & $5.20 \mathrm{e}-2$ & 0.63 \\
\hline & 40 & * & * & $4.17 \mathrm{e}-2$ & 0.15 & $4.00 \mathrm{e}-2$ & 0.31 & $4.20 \mathrm{e}-2$ & 0.28 & $4.21 \mathrm{e}-2$ & 0.30 \\
\hline & 80 & * & * & $2.73 \mathrm{e}-2$ & 0.61 & $2.10 \mathrm{e}-2$ & 0.93 & $2.14 \mathrm{e}-2$ & 0.98 & $2.18 \mathrm{e}-2$ & 0.95 \\
\hline & 160 & * & * & $*$ & * & $2.05 \mathrm{e}-2$ & 0.04 & $2.09 \mathrm{e}-2$ & 0.03 & $2.11 \mathrm{e}-2$ & 0.04 \\
\hline & 320 & * & * & * & * & $1.27 \mathrm{e}-2$ & 0.69 & $1.41 \mathrm{e}-2$ & 0.57 & $1.45 \mathrm{e}-2$ & 0.54 \\
\hline 3 & 10 & * & & $*$ & & * & & $*$ & & $6.02 \mathrm{e}-2$ & \\
\hline & 20 & * & * & * & $*$ & * & $*$ & * & $*$ & * & * \\
\hline & 40 & * & * & * & $*$ & * & * & * & * & * & * \\
\hline & 80 & * & * & * & * & * & * & * & * & * & * \\
\hline & 160 & * & * & * & * & * & * & * & * & * & * \\
\hline & 320 & * & * & $*$ & $*$ & * & $*$ & * & $*$ & * & * \\
\hline 4 & 10 & * & & * & & * & & $*$ & & * & \\
\hline & 20 & * & * & * & $*$ & * & $*$ & * & $*$ & $*$ & * \\
\hline & 40 & * & * & * & * & * & * & * & * & * & * \\
\hline & 80 & * & * & * & * & * & * & * & $*$ & * & * \\
\hline & 160 & * & $*$ & $*$ & $*$ & * & * & * & $*$ & * & * \\
\hline & 320 & * & $*$ & $*$ & $*$ & * & * & * & $*$ & * & * \\
\hline 5 & 10 & * & & $*$ & & * & & * & & $*$ & \\
\hline & 20 & * & $*$ & $*$ & $*$ & * & $*$ & * & $*$ & * & * \\
\hline & 40 & * & $*$ & $*$ & $*$ & * & $*$ & * & $*$ & * & $*$ \\
\hline & 80 & * & * & $*$ & $*$ & * & $*$ & * & $*$ & * & * \\
\hline & 160 & $*$ & $*$ & $*$ & * & * & * & * & $*$ & $*$ & $*$ \\
\hline & 320 & * & $*$ & $*$ & $*$ & * & * & * & $*$ & * & * \\
\hline
\end{tabular}


Table 9: Errors and experimental order of convergence (EOC) for varying polynomial degrees $p$ and number of elements $N$ for the modified Sod shock tube problem (128) using the local LaxFriedrichs numerical flux and "simple" volume fluxes.

\begin{tabular}{|c|c|c|c|c|c|c|c|c|c|c|c|}
\hline \multirow[b]{2}{*}{$p$} & \multirow[b]{2}{*}{$N$} & \multicolumn{2}{|c|}{ Central } & \multicolumn{2}{|c|}{ Morinishi } & \multicolumn{2}{|c|}{ Ducros } & \multicolumn{2}{|c|}{$\mathrm{KG}$} & \multicolumn{2}{|c|}{ Pirozzoli } \\
\hline & & $\left\|\operatorname{err}_{\varrho}\right\|_{M}$ & EOC & $\left\|\operatorname{err}_{\varrho}\right\|_{M}$ & EOC & $\left\|\operatorname{err}_{\varrho}\right\|_{M}$ & EOC & $\left\|\operatorname{err}_{\varrho}\right\|_{M}$ & EOC & $\left\|\operatorname{err}_{\varrho}\right\|_{M}$ & EOC \\
\hline \multirow[t]{6}{*}{1} & 10 & $1.44 \mathrm{e}-1$ & & $1.45 \mathrm{e}-1$ & & $1.35 \mathrm{e}-1$ & & $1.34 \mathrm{e}-1$ & & $1.34 \mathrm{e}-1$ & \\
\hline & 20 & $7.30 \mathrm{e}-2$ & 0.98 & $8.23 \mathrm{e}-2$ & 0.81 & $6.95 \mathrm{e}-2$ & 0.96 & $7.18 \mathrm{e}-2$ & 0.90 & $7.23 \mathrm{e}-2$ & 0.89 \\
\hline & 40 & $6.68 \mathrm{e}-2$ & 0.13 & $9.44 \mathrm{e}-2$ & -0.20 & $5.91 \mathrm{e}-2$ & 0.23 & $6.30 \mathrm{e}-2$ & 0.19 & $6.44 \mathrm{e}-2$ & 0.17 \\
\hline & 80 & $6.08 \mathrm{e}-2$ & 0.13 & $*$ & $*$ & $4.62 \mathrm{e}-2$ & 0.36 & $4.94 \mathrm{e}-2$ & 0.35 & $5.04 \mathrm{e}-2$ & 0.35 \\
\hline & 160 & $5.16 \mathrm{e}-2$ & 0.24 & * & $*$ & $3.22 \mathrm{e}-2$ & 0.52 & $3.48 \mathrm{e}-2$ & 0.50 & $3.58 \mathrm{e}-2$ & 0.49 \\
\hline & 320 & $4.72 \mathrm{e}-2$ & 0.13 & * & * & $2.25 \mathrm{e}-2$ & 0.52 & $2.46 \mathrm{e}-2$ & 0.50 & $2.53 \mathrm{e}-2$ & 0.50 \\
\hline \multirow[t]{6}{*}{2} & 10 & $8.42 \mathrm{e}-2$ & & $8.46 \mathrm{e}-2$ & & $8.62 \mathrm{e}-2$ & & $8.67 \mathrm{e}-2$ & & $8.67 \mathrm{e}-2$ & \\
\hline & 20 & $5.08 \mathrm{e}-2$ & 0.73 & $5.23 \mathrm{e}-2$ & 0.69 & $5.36 \mathrm{e}-2$ & 0.68 & $5.43 \mathrm{e}-2$ & 0.68 & $5.50 \mathrm{e}-2$ & 0.66 \\
\hline & 40 & $3.78 \mathrm{e}-2$ & 0.42 & $4.92 \mathrm{e}-2$ & 0.09 & $3.99 \mathrm{e}-2$ & 0.43 & $4.20 \mathrm{e}-2$ & 0.37 & $4.22 \mathrm{e}-2$ & 0.38 \\
\hline & 80 & $2.30 \mathrm{e}-2$ & 0.72 & $*$ & * & $2.25 \mathrm{e}-2$ & 0.82 & $2.21 \mathrm{e}-2$ & 0.93 & $2.25 \mathrm{e}-2$ & 0.90 \\
\hline & 160 & $2.14 \mathrm{e}-2$ & 0.10 & * & $*$ & $2.11 \mathrm{e}-2$ & 0.10 & $2.08 \mathrm{e}-2$ & 0.09 & $2.10 \mathrm{e}-2$ & 0.10 \\
\hline & 320 & $1.43 \mathrm{e}-2$ & 0.59 & $*$ & $*$ & $1.32 \mathrm{e}-2$ & 0.67 & $1.40 \mathrm{e}-2$ & 0.56 & $1.44 \mathrm{e}-2$ & 0.54 \\
\hline \multirow[t]{6}{*}{3} & 10 & $*$ & & $*$ & & $*$ & & $6.11 \mathrm{e}-2$ & & $6.22 \mathrm{e}-2$ & \\
\hline & 20 & * & $*$ & $*$ & * & $*$ & $*$ & $4.53 \mathrm{e}-2$ & 0.43 & $4.58 \mathrm{e}-2$ & 0.44 \\
\hline & 40 & * & * & $*$ & $*$ & $*$ & * & $2.48 \mathrm{e}-2$ & 0.87 & $2.51 \mathrm{e}-2$ & 0.87 \\
\hline & 80 & * & * & $*$ & $*$ & $*$ & $*$ & $2.28 \mathrm{e}-2$ & 0.12 & $2.29 \mathrm{e}-2$ & 0.13 \\
\hline & 160 & $*$ & $*$ & $*$ & $*$ & $*$ & $*$ & $1.52 \mathrm{e}-2$ & 0.58 & $1.55 \mathrm{e}-2$ & 0.57 \\
\hline & 320 & $*$ & $*$ & * & $*$ & $*$ & $*$ & $1.16 \mathrm{e}-2$ & 0.39 & $1.17 \mathrm{e}-2$ & 0.40 \\
\hline \multirow[t]{6}{*}{4} & 10 & * & & $*$ & & * & & * & & * & \\
\hline & 20 & * & * & * & * & * & * & * & $*$ & * & * \\
\hline & 40 & * & * & $*$ & * & * & $*$ & * & * & * & * \\
\hline & 80 & $*$ & $*$ & $*$ & $*$ & $*$ & $*$ & $*$ & $*$ & $*$ & * \\
\hline & 160 & * & $*$ & $*$ & * & $*$ & * & * & * & * & * \\
\hline & 320 & $*$ & $*$ & $*$ & $*$ & $*$ & $*$ & $*$ & $*$ & $*$ & * \\
\hline \multirow[t]{6}{*}{5} & 10 & $*$ & & $*$ & & $*$ & & $*$ & & * & \\
\hline & 20 & * & * & $*$ & * & $*$ & * & * & * & * & * \\
\hline & 40 & * & $*$ & $*$ & $*$ & $*$ & $*$ & $*$ & $*$ & * & * \\
\hline & 80 & * & $*$ & $*$ & $*$ & $*$ & $*$ & $*$ & $*$ & * & * \\
\hline & 160 & * & * & * & * & * & * & * & * & * & * \\
\hline & 320 & $*$ & $*$ & $*$ & $*$ & $*$ & * & * & * & * & * \\
\hline
\end{tabular}




\subsection{Sod's Shock Tube: Finite Volume Setting}

Here, the classical shock tube of Sod [24] with initial condition (127) of section 7.2 will be used again, but in the context of first order finite volume methods.

The entropy conservative flux (52) of Chandrashekar [3] has been used with the scalar dissipation (SD) of Derigs, Winters, Gassner, and Walch [6], the matrix (MD) and hybrid (HD) dissipation of Winters, Derigs, Gassner, and Walch [28] and the local Lax-Friedrichs (LLF) dissipation operator. The last one has also been used for the other entropy conservative fluxes. Additionally, the classical LLF flux and Suliciu relaxation solver of Bouchut [2] are tested.

The results are shown in Table 10. Here, the matrix dissipation (MD) and the Suliciu solver perform equally good and yield less error than the other fluxes. Additionally, there is nearly no variance across the solvers using the LLF or scalar dissipation operator.

Table 10: Errors and experimental order of convergence (EOC) for varying number of elements $N$ for the Sod shock tube problem (127) using several numerical fluxes.

\begin{tabular}{|c|c|c|c|c|c|c|c|c|}
\hline \multirow[b]{2}{*}{$N$} & \multicolumn{2}{|c|}{$\mathrm{Ch}+$ SD DWGW } & \multicolumn{2}{|c|}{$\mathrm{Ch}+\mathrm{MD}$ DWGW } & \multicolumn{2}{|c|}{$\mathrm{Ch}+\mathrm{HD}$ DWGW } & \multicolumn{2}{|c|}{$\mathrm{Ch}+\mathrm{LLF}$} \\
\hline & $\left\|\operatorname{err}_{\varrho}\right\|_{M}$ & EOC & $\left\|\operatorname{err}_{\varrho}\right\|_{M}$ & EOC & $\left\|\operatorname{err}_{\varrho}\right\|_{M}$ & EOC & $\left\|\operatorname{err}_{\varrho}\right\|_{M}$ & EOC \\
\hline 100 & $4.62 \mathrm{e}-2$ & & $3.97 \mathrm{e}-2$ & & $4.22 \mathrm{e}-2$ & & $4.62 \mathrm{e}-2$ & \\
\hline 200 & $3.75 \mathrm{e}-2$ & 0.30 & $3.04 \mathrm{e}-2$ & 0.38 & $3.23 \mathrm{e}-2$ & 0.38 & $3.75 \mathrm{e}-2$ & 0.30 \\
\hline 400 & $2.80 \mathrm{e}-2$ & 0.42 & $2.19 \mathrm{e}-2$ & 0.47 & $2.31 \mathrm{e}-2$ & 0.49 & $2.80 \mathrm{e}-2$ & 0.42 \\
\hline 800 & $2.08 \mathrm{e}-2$ & 0.43 & $1.62 \mathrm{e}-2$ & 0.43 & $1.69 \mathrm{e}-2$ & 0.45 & $2.08 \mathrm{e}-2$ & 0.43 \\
\hline 1600 & $1.56 \mathrm{e}-2$ & 0.42 & $1.23 \mathrm{e}-2$ & 0.40 & $1.26 \mathrm{e}-2$ & 0.42 & $1.56 \mathrm{e}-2$ & 0.42 \\
\hline 3200 & $1.18 \mathrm{e}-2$ & 0.40 & $9.33 \mathrm{e}-3$ & 0.40 & $9.49 \mathrm{e}-3$ & 0.41 & $1.18 \mathrm{e}-2$ & 0.40 \\
\hline 6400 & $9.24 \mathrm{e}-3$ & 0.35 & $7.35 \mathrm{e}-3$ & 0.34 & $7.42 \mathrm{e}-3$ & 0.36 & $9.24 \mathrm{e}-3$ & 0.35 \\
\hline \multirow[t]{2}{*}{12800} & $7.22 \mathrm{e}-3$ & 0.36 & $5.60 \mathrm{e}-3$ & 0.39 & $5.64 \mathrm{e}-3$ & 0.40 & $7.22 \mathrm{e}-3$ & 0.36 \\
\hline & \multicolumn{2}{|c|}{$\varrho, v, \beta(2)+\mathrm{LLF}$} & \multicolumn{2}{|c|}{$\varrho, v, \frac{1}{n}+\mathrm{LLF}$} & \multicolumn{2}{|c|}{$\varrho, v, p+\mathrm{LLF}$} & \multicolumn{2}{|c|}{$\varrho, v, T(1)+\mathrm{LLF}$} \\
\hline$N$ & $\left\|\operatorname{err}_{\varrho}\right\|_{M}$ & EOC & $\left\|\operatorname{err}_{\varrho}\right\|_{M}$ & EOC & $\left\|\operatorname{err}_{\varrho}\right\|_{M}$ & EOC & $\left\|\operatorname{err}_{\varrho}\right\|_{M}$ & EOC \\
\hline 100 & $4.62 \mathrm{e}-2$ & & $4.61 \mathrm{e}-2$ & & $4.61 \mathrm{e}-2$ & & $4.62 \mathrm{e}-2$ & \\
\hline 200 & $3.75 \mathrm{e}-2$ & 0.30 & $3.74 \mathrm{e}-2$ & 0.30 & $3.74 \mathrm{e}-2$ & 0.30 & $3.75 \mathrm{e}-2$ & 0.30 \\
\hline 400 & $2.80 \mathrm{e}-2$ & 0.42 & $2.80 \mathrm{e}-2$ & 0.42 & $2.80 \mathrm{e}-2$ & 0.42 & $2.80 \mathrm{e}-2$ & 0.42 \\
\hline 800 & $2.08 \mathrm{e}-2$ & 0.43 & $2.08 \mathrm{e}-2$ & 0.43 & $2.08 \mathrm{e}-2$ & 0.43 & $2.08 \mathrm{e}-2$ & 0.43 \\
\hline 1600 & $1.56 \mathrm{e}-2$ & 0.42 & $1.56 \mathrm{e}-2$ & 0.42 & $1.56 \mathrm{e}-2$ & 0.42 & $1.56 \mathrm{e}-2$ & 0.42 \\
\hline 3200 & $1.18 \mathrm{e}-2$ & 0.40 & $1.18 \mathrm{e}-2$ & 0.40 & $1.18 \mathrm{e}-2$ & 0.40 & $1.18 \mathrm{e}-2$ & 0.40 \\
\hline 6400 & $9.24 \mathrm{e}-3$ & 0.35 & $9.24 \mathrm{e}-3$ & 0.35 & $9.24 \mathrm{e}-3$ & 0.35 & $9.24 \mathrm{e}-3$ & 0.35 \\
\hline \multirow[t]{2}{*}{12800} & $7.22 \mathrm{e}-3$ & 0.36 & $7.22 \mathrm{e}-3$ & 0.36 & $7.22 \mathrm{e}-3$ & 0.36 & $7.22 \mathrm{e}-3$ & 0.36 \\
\hline & \multicolumn{2}{|c|}{$\varrho, v, T(2)+\mathrm{LLF}$} & \multicolumn{2}{|c|}{$\varrho, V, T$ (rev) $+\mathrm{LLF}$} & \multicolumn{2}{|c|}{ LLF } & \multicolumn{2}{|c|}{ Suliciu } \\
\hline$N$ & $\left\|\operatorname{err}_{\varrho}\right\|_{M}$ & EOC & $\left\|\operatorname{err}_{\varrho}\right\|_{M}$ & EOC & $\left\|\operatorname{err}_{\varrho}\right\|_{M}$ & EOC & $\left\|\operatorname{err}_{\varrho}\right\|_{M}$ & EOC \\
\hline 100 & $4.62 \mathrm{e}-2$ & & $4.63 \mathrm{e}-2$ & & $4.58 \mathrm{e}-2$ & & $3.95 \mathrm{e}-2$ & \\
\hline 200 & $3.75 \mathrm{e}-2$ & 0.30 & $3.76 \mathrm{e}-2$ & 0.30 & $3.72 \mathrm{e}-2$ & 0.30 & $3.03 \mathrm{e}-2$ & 0.38 \\
\hline 400 & $2.80 \mathrm{e}-2$ & 0.42 & $2.80 \mathrm{e}-2$ & 0.42 & $2.79 \mathrm{e}-2$ & 0.42 & $2.19 \mathrm{e}-2$ & 0.47 \\
\hline 800 & $2.08 \mathrm{e}-2$ & 0.43 & $2.08 \mathrm{e}-2$ & 0.43 & $2.07 \mathrm{e}-2$ & 0.43 & $1.63 \mathrm{e}-2$ & 0.43 \\
\hline 1600 & $1.56 \mathrm{e}-2$ & 0.42 & $1.56 \mathrm{e}-2$ & 0.42 & $1.56 \mathrm{e}-2$ & 0.41 & $1.23 \mathrm{e}-2$ & 0.40 \\
\hline 3200 & $1.18 \mathrm{e}-2$ & 0.40 & $1.18 \mathrm{e}-2$ & 0.40 & $1.18 \mathrm{e}-2$ & 0.40 & $9.36 \mathrm{e}-3$ & 0.39 \\
\hline 6400 & $9.24 \mathrm{e}-3$ & 0.35 & $9.24 \mathrm{e}-3$ & 0.35 & $9.25 \mathrm{e}-3$ & 0.35 & $7.36 \mathrm{e}-3$ & 0.35 \\
\hline 12800 & $7.22 \mathrm{e}-3$ & 0.36 & $7.22 \mathrm{e}-3$ & 0.36 & $7.23 \mathrm{e}-3$ & 0.36 & $5.63 \mathrm{e}-3$ & 0.39 \\
\hline
\end{tabular}

\subsection{Modified Version of Sod's Shock Tube: Finite Volume Setting}

Similar to the previous section, the modified Sod shock tube problem of section 7.3 is used to test the finite volume fluxes. The results are shown in Table 11. Again, the matrix dissipation and the Suliciu solver perform equally good and are superior to the other fluxes. As in section 7.4, there is nearly no variance across the methods with matrix / LLF dissipation. 
Table 11: Errors and experimental order of convergence (EOC) for varying number of elements $N$ for the modified Sod shock tube problem (128) using several numerical fluxes.

\begin{tabular}{|c|c|c|c|c|c|c|c|c|}
\hline \multirow[b]{2}{*}{$N$} & \multicolumn{2}{|c|}{$\mathrm{Ch}+\mathrm{SD} \mathrm{DWGW}$} & \multicolumn{2}{|c|}{$\mathrm{Ch}+\mathrm{MD} \mathrm{DWGW}$} & \multicolumn{2}{|c|}{$\mathrm{Ch}+\mathrm{HD} \mathrm{DWGW}$} & \multicolumn{2}{|c|}{$\mathrm{Ch}+\mathrm{LLF}$} \\
\hline & $\left\|\operatorname{err}_{\varrho}\right\|_{M}$ & $\mathrm{EOC}$ & $\left\|\operatorname{err}_{\varrho}\right\|_{M}$ & $\mathrm{EOC}$ & $\left\|\operatorname{err}_{\varrho}\right\|_{M}$ & EOC & $\left\|\operatorname{err}_{\varrho}\right\|_{M}$ & $\mathrm{EOC}$ \\
\hline 100 & $5.55 \mathrm{e}-2$ & & $4.03 \mathrm{e}-2$ & & $4.59 \mathrm{e}-2$ & & $5.55 \mathrm{e}-2$ & \\
\hline 200 & $4.67 \mathrm{e}-2$ & 0.25 & $3.31 \mathrm{e}-2$ & 0.28 & $3.61 \mathrm{e}-2$ & 0.35 & $4.67 \mathrm{e}-2$ & 0.25 \\
\hline 400 & $3.70 \mathrm{e}-2$ & 0.34 & $2.65 \mathrm{e}-2$ & 0.32 & $2.80 \mathrm{e}-2$ & 0.37 & $3.70 \mathrm{e}-2$ & 0.34 \\
\hline 800 & $2.90 \mathrm{e}-2$ & 0.35 & $2.14 \mathrm{e}-2$ & 0.31 & $2.21 \mathrm{e}-2$ & 0.34 & $2.90 \mathrm{e}-2$ & 0.35 \\
\hline 1600 & $2.23 \mathrm{e}-2$ & 0.38 & $1.68 \mathrm{e}-2$ & 0.35 & $1.72 \mathrm{e}-2$ & 0.37 & $2.23 \mathrm{e}-2$ & 0.38 \\
\hline 3200 & $1.76 \mathrm{e}-2$ & 0.34 & $1.37 \mathrm{e}-2$ & 0.29 & $1.39 \mathrm{e}-2$ & 0.31 & $1.76 \mathrm{e}-2$ & 0.34 \\
\hline 6400 & $1.39 \mathrm{e}-2$ & 0.34 & $1.10 \mathrm{e}-2$ & 0.32 & $1.11 \mathrm{e}-2$ & 0.32 & $1.39 \mathrm{e}-2$ & 0.34 \\
\hline 12800 & $1.10 \mathrm{e}-2$ & 0.34 & $8.51 \mathrm{e}-3$ & 0.37 & $8.57 \mathrm{e}-3$ & 0.37 & $1.10 \mathrm{e}-2$ & 0.34 \\
\hline \multirow[b]{2}{*}{$N$} & \multicolumn{2}{|c|}{$\varrho, v, \beta(2)+\mathrm{LLF}$} & \multicolumn{2}{|c|}{$\varrho, v, \frac{1}{p}+\mathrm{LLF}$} & \multicolumn{2}{|c|}{$\varrho, v, p+\mathrm{LLF}$} & \multicolumn{2}{|c|}{$\varrho, v, T(1)+\mathrm{LLF}$} \\
\hline & $\left\|\operatorname{err}_{\varrho}\right\|_{M}$ & $\mathrm{EOC}$ & $\mid \operatorname{err}_{\varrho} \|_{M}$ & EOC & $\mid \operatorname{err}_{\varrho} \|_{M}$ & $\mathrm{EOC}$ & $\left\|\operatorname{err}_{\varrho}\right\|_{M}$ & EOC \\
\hline 100 & $5.55 \mathrm{e}-2$ & & $5.53 \mathrm{e}-2$ & & $5.53 \mathrm{e}-2$ & & $5.55 \mathrm{e}-2$ & \\
\hline 200 & $4.67 \mathrm{e}-2$ & 0.25 & $4.67 \mathrm{e}-2$ & 0.25 & $4.67 \mathrm{e}-2$ & 0.25 & $4.67 \mathrm{e}-2$ & 0.25 \\
\hline 400 & $3.70 \mathrm{e}-2$ & 0.34 & $3.70 \mathrm{e}-2$ & 0.33 & $3.70 \mathrm{e}-2$ & 0.33 & $3.70 \mathrm{e}-2$ & 0.34 \\
\hline 800 & $2.90 \mathrm{e}-2$ & 0.35 & $2.90 \mathrm{e}-2$ & 0.35 & $2.90 \mathrm{e}-2$ & 0.35 & $2.90 \mathrm{e}-2$ & 0.35 \\
\hline 1600 & $2.23 \mathrm{e}-2$ & 0.38 & $2.23 \mathrm{e}-2$ & 0.38 & $2.23 \mathrm{e}-2$ & 0.38 & $2.23 \mathrm{e}-2$ & 0.38 \\
\hline 3200 & $1.76 \mathrm{e}-2$ & 0.34 & $1.76 \mathrm{e}-2$ & 0.34 & $1.76 \mathrm{e}-2$ & 0.34 & $1.76 \mathrm{e}-2$ & 0.34 \\
\hline 6400 & $1.39 \mathrm{e}-2$ & 0.34 & $1.39 \mathrm{e}-2$ & 0.34 & $1.39 \mathrm{e}-2$ & 0.34 & $1.39 \mathrm{e}-2$ & 0.34 \\
\hline \multirow[t]{2}{*}{12800} & $1.10 \mathrm{e}-2$ & 0.34 & $1.10 \mathrm{e}-2$ & 0.34 & $1.10 \mathrm{e}-2$ & 0.34 & $1.10 \mathrm{e}-2$ & 0.34 \\
\hline & \multicolumn{2}{|c|}{$\varrho, v, T(2)+\mathrm{LLF}$} & \multicolumn{2}{|c|}{$\varrho, V, T(\mathrm{rev})+\mathrm{LLF}$} & \multicolumn{2}{|c|}{ LLF } & \multicolumn{2}{|c|}{ Suliciu } \\
\hline$N$ & $\left\|\operatorname{err}_{\varrho}\right\|_{M}$ & EOC & $\left\|\operatorname{err}_{\varrho}\right\|_{M}$ & EOC & $\left\|\operatorname{err}_{\varrho}\right\|_{M}$ & EOC & $\left\|\operatorname{err}_{\varrho}\right\|_{M}$ & EOC \\
\hline 100 & $5.55 \mathrm{e}-2$ & & $5.55 \mathrm{e}-2$ & & $5.58 \mathrm{e}-2$ & & $4.13 \mathrm{e}-2$ & \\
\hline 200 & $4.67 \mathrm{e}-2$ & 0.25 & $4.67 \mathrm{e}-2$ & 0.25 & $4.69 \mathrm{e}-2$ & 0.25 & $3.34 \mathrm{e}-2$ & 0.31 \\
\hline 400 & $3.70 \mathrm{e}-2$ & 0.34 & $3.70 \mathrm{e}-2$ & 0.34 & $3.71 \mathrm{e}-2$ & 0.34 & $2.66 \mathrm{e}-2$ & 0.33 \\
\hline 800 & $2.90 \mathrm{e}-2$ & 0.35 & $2.90 \mathrm{e}-2$ & 0.35 & $2.91 \mathrm{e}-2$ & 0.35 & $2.15 \mathrm{e}-2$ & 0.31 \\
\hline 1600 & $2.23 \mathrm{e}-2$ & 0.38 & $2.23 \mathrm{e}-2$ & 0.38 & $2.24 \mathrm{e}-2$ & 0.38 & $1.69 \mathrm{e}-2$ & 0.35 \\
\hline 3200 & $1.76 \mathrm{e}-2$ & 0.34 & $1.76 \mathrm{e}-2$ & 0.34 & $1.76 \mathrm{e}-2$ & 0.34 & $1.38 \mathrm{e}-2$ & 0.29 \\
\hline 6400 & $1.39 \mathrm{e}-2$ & 0.34 & $1.39 \mathrm{e}-2$ & 0.34 & $1.40 \mathrm{e}-2$ & 0.34 & $1.11 \mathrm{e}-2$ & 0.32 \\
\hline 12800 & $1.10 \mathrm{e}-2$ & 0.34 & $1.10 \mathrm{e}-2$ & 0.34 & $1.10 \mathrm{e}-2$ & 0.34 & $8.61 \mathrm{e}-3$ & 0.36 \\
\hline
\end{tabular}




\subsection{Near Vacuum Rarefaction}

In this section, the rarefaction waves near vacuum as described by Toro [27, Section 4.3.3, Test 2 ] will be used to test the methods. The initial condition is given in primitive variables by

$$
\varrho_{0}(x)=1, \quad v_{0}(x)=\left\{\begin{array}{ll}
-2, & x<\frac{1}{2}, \\
2, & \text { else },
\end{array}, \quad p_{0}(x)=0.4,\right.
$$

and the conservative variables are again computed via $\varrho v_{0}=\varrho_{0} v_{0}$ and $\varrho e_{0}=\frac{1}{2} \varrho_{0} v_{0}^{2}+\frac{p_{0}}{\gamma-1}$. The solution is computed on the domain $[0,1]$ from $t=0$ until $t=0.15$.

Using the same finite volume methods as in section 7.4, the results are shown in Table 12. Across the varying number of elements $N$, no flux is clearly superior.

Table 12: Errors and experimental order of convergence (EOC) for varying number of elements $N$ for the near vacuum rarefaction problem (129) using several numerical fluxes.

\begin{tabular}{|c|c|c|c|c|c|c|c|c|}
\hline \multirow[b]{2}{*}{$N$} & \multicolumn{2}{|c|}{$\mathrm{Ch}+\mathrm{SD}$ DWGW } & \multicolumn{2}{|c|}{ Ch + MD DWGW } & \multicolumn{2}{|c|}{$\mathrm{Ch}+\mathrm{HD}$ DWGW } & \multicolumn{2}{|c|}{$\mathrm{Ch}+\mathrm{LLF}$} \\
\hline & $\left\|\operatorname{err}_{\varrho}\right\|_{M}$ & $\mathrm{EOC}$ & $\left\|\operatorname{err}_{\varrho}\right\|_{M}$ & EOC & $\left\|\operatorname{err}_{\varrho}\right\|_{M}$ & $\mathrm{EOC}$ & $\left\|\operatorname{err}_{\varrho}\right\|_{M}$ & EOC \\
\hline 100 & $8.83 \mathrm{e}-2$ & & $7.94 \mathrm{e}-2$ & & $7.98 \mathrm{e}-2$ & & $8.84 \mathrm{e}-2$ & \\
\hline 200 & $5.66 \mathrm{e}-2$ & 0.64 & $5.67 \mathrm{e}-2$ & 0.49 & $5.62 \mathrm{e}-2$ & 0.51 & $5.66 \mathrm{e}-2$ & 0.64 \\
\hline 400 & $4.01 \mathrm{e}-2$ & 0.50 & $4.18 \mathrm{e}-2$ & 0.44 & $4.14 \mathrm{e}-2$ & 0.44 & $4.01 \mathrm{e}-2$ & 0.50 \\
\hline 800 & $2.91 \mathrm{e}-2$ & 0.46 & $2.99 \mathrm{e}-2$ & 0.48 & $2.98 \mathrm{e}-2$ & 0.47 & $2.91 \mathrm{e}-2$ & 0.46 \\
\hline 1600 & $2.02 \mathrm{e}-2$ & 0.52 & $2.05 \mathrm{e}-2$ & 0.54 & $2.05 \mathrm{e}-2$ & 0.54 & $2.03 \mathrm{e}-2$ & 0.52 \\
\hline 3200 & $1.32 \mathrm{e}-2$ & 0.61 & $1.33 \mathrm{e}-2$ & 0.62 & $1.33 \mathrm{e}-2$ & 0.62 & $1.33 \mathrm{e}-2$ & 0.61 \\
\hline 6400 & $8.04 \mathrm{e}-3$ & 0.72 & $8.08 \mathrm{e}-3$ & 0.72 & $8.06 \mathrm{e}-3$ & 0.72 & $8.04 \mathrm{e}-3$ & 0.72 \\
\hline \multirow[t]{2}{*}{12800} & $4.25 \mathrm{e}-3$ & 0.92 & $4.22 \mathrm{e}-3$ & 0.94 & $4.20 \mathrm{e}-3$ & 0.94 & $4.26 \mathrm{e}-3$ & 0.92 \\
\hline & \multicolumn{2}{|c|}{$\varrho, v, \beta(2)+\mathrm{LLF}$} & \multicolumn{2}{|c|}{$\varrho, v, \frac{1}{p}+\mathrm{LLF}$} & \multicolumn{2}{|c|}{$\varrho, v, p+\mathrm{LLF}$} & \multicolumn{2}{|c|}{$\varrho, v, T(1)+\mathrm{LLF}$} \\
\hline$N$ & $\left\|\operatorname{err}_{\varrho}\right\|_{M}$ & EOC & $\left\|\operatorname{err}_{\varrho}\right\|_{M}^{P}$ & EOC & $\left\|\operatorname{err}_{\varrho}\right\|_{M}$ & $\mathrm{EOC}$ & $\left\|\operatorname{err}_{\varrho}\right\|_{M}$ & EOC \\
\hline 100 & $8.80 \mathrm{e}-2$ & & $8.87 \mathrm{e}-2$ & & $8.87 \mathrm{e}-2$ & & $8.84 \mathrm{e}-2$ & \\
\hline 200 & $5.65 \mathrm{e}-2$ & 0.64 & $5.68 \mathrm{e}-2$ & 0.64 & $5.68 \mathrm{e}-2$ & 0.64 & $5.66 \mathrm{e}-2$ & 0.64 \\
\hline 400 & $4.01 \mathrm{e}-2$ & 0.49 & $4.04 \mathrm{e}-2$ & 0.49 & $4.04 \mathrm{e}-2$ & 0.49 & $4.01 \mathrm{e}-2$ & 0.50 \\
\hline 800 & $2.91 \mathrm{e}-2$ & 0.46 & $2.93 \mathrm{e}-2$ & 0.46 & $2.93 \mathrm{e}-2$ & 0.46 & $2.91 \mathrm{e}-2$ & 0.46 \\
\hline 1600 & $2.03 \mathrm{e}-2$ & 0.52 & $2.03 \mathrm{e}-2$ & 0.53 & $2.03 \mathrm{e}-2$ & 0.53 & $2.03 \mathrm{e}-2$ & 0.52 \\
\hline 3200 & $1.32 \mathrm{e}-2$ & 0.61 & $1.33 \mathrm{e}-2$ & 0.61 & $1.33 \mathrm{e}-2$ & 0.61 & $1.33 \mathrm{e}-2$ & 0.61 \\
\hline 6400 & $8.04 \mathrm{e}-3$ & 0.72 & $8.07 \mathrm{e}-3$ & 0.72 & $8.07 \mathrm{e}-3$ & 0.72 & $8.04 \mathrm{e}-3$ & 0.72 \\
\hline \multirow[t]{2}{*}{12800} & $4.25 \mathrm{e}-3$ & 0.92 & $4.29 \mathrm{e}-3$ & 0.91 & $4.29 \mathrm{e}-3$ & 0.91 & $4.26 \mathrm{e}-3$ & 0.92 \\
\hline & \multicolumn{2}{|c|}{$\varrho, v, T(2)+\mathrm{LLF}$} & \multicolumn{2}{|c|}{$\varrho, V, T(\mathrm{rev})+\mathrm{LLF}$} & \multicolumn{2}{|c|}{ LLF } & \multicolumn{2}{|c|}{ Suliciu } \\
\hline$N$ & $\left\|\operatorname{err}_{\varrho}\right\|_{M}$ & $\mathrm{EOC}$ & $\left\|\operatorname{err}_{\varrho}\right\|_{M}$ & $\mathrm{EOC}$ & $\left\|\operatorname{err}_{\varrho}\right\|_{M}$ & $\mathrm{EOC}$ & $\left\|\operatorname{err}_{\varrho}\right\|_{M}$ & EOC \\
\hline 100 & $8.80 \mathrm{e}-2$ & & $8.84 \mathrm{e}-2$ & & $8.07 \mathrm{e}-2$ & & $7.72 \mathrm{e}-2$ & \\
\hline 200 & $5.63 \mathrm{e}-2$ & 0.64 & $5.67 \mathrm{e}-2$ & 0.64 & $5.52 \mathrm{e}-2$ & 0.55 & $5.55 \mathrm{e}-2$ & 0.47 \\
\hline 400 & $3.99 \mathrm{e}-2$ & 0.50 & $4.02 \mathrm{e}-2$ & 0.50 & $4.00 \mathrm{e}-2$ & 0.46 & $4.10 \mathrm{e}-2$ & 0.44 \\
\hline 800 & $2.90 \mathrm{e}-2$ & 0.46 & $2.92 \mathrm{e}-2$ & 0.46 & $2.89 \mathrm{e}-2$ & 0.47 & $2.95 \mathrm{e}-2$ & 0.48 \\
\hline 1600 & $2.02 \mathrm{e}-2$ & 0.52 & $2.03 \mathrm{e}-2$ & 0.52 & $2.01 \mathrm{e}-2$ & 0.53 & $2.02 \mathrm{e}-2$ & 0.54 \\
\hline 3200 & $1.32 \mathrm{e}-2$ & 0.61 & $1.33 \mathrm{e}-2$ & 0.61 & $1.31 \mathrm{e}-2$ & 0.61 & $1.32 \mathrm{e}-2$ & 0.62 \\
\hline 6400 & $8.02 \mathrm{e}-3$ & 0.72 & $8.05 \mathrm{e}-3$ & 0.72 & $7.92 \mathrm{e}-3$ & 0.73 & $7.98 \mathrm{e}-3$ & 0.72 \\
\hline 12800 & $4.23 \mathrm{e}-3$ & 0.92 & $4.26 \mathrm{e}-3$ & 0.92 & $4.05 \mathrm{e}-3$ & 0.97 & $4.12 \mathrm{e}-3$ & 0.95 \\
\hline
\end{tabular}

\subsection{Left Half of the Blast Wave Problem of Woodward and Colella}

In this section, the left half of the blast wave problem of Woodward and Colella [29, Section IV.a] as described by Toro [27, Section 4.3.3, Test 3] is considered. In primitive variables, it is given by

$$
\varrho_{0}(x)=1, \quad v_{0}(x)=0, \quad p_{0}(x)= \begin{cases}1000, & x<\frac{1}{2}, \\ 0.01, & \text { else }\end{cases}
$$


and the conservative variables are again computed via $\varrho v_{0}=\varrho_{0} v_{0}$ and $\varrho e_{0}=\frac{1}{2} \varrho_{0} v_{0}^{2}+\frac{p_{0}}{\gamma-1}$. The solution is computed on the domain $[0,1]$ from $t=0$ until $t=0.012$ using the finite volume methods described in section 7.4.

The results are shown in table 13. The simulations using the fluxes with variables $\varrho, v$ and $p$ or $\frac{1}{p}$ crashed since they left the invariant region for the Euler equations. As described by Derigs, Winters, Gassner, and Walch [6], the reason is the appearance of the pressure in the density flux as in the entropy conservative flux of Roe [22].

The Suliciu solver and the matrix dissipation (MD) yielded similar errors until the last one crashed using 12800 elements. There is a bit more variance across the other fluxes than in the previous sections 7.4, 7.5 and 7.6. However, in the end, the Suliciu relaxation solver performs better than the others.

Table 13: Errors and experimental order of convergence (EOC) for varying number of elements $N$ for the left half (130) of the blast wave problem of Woodward and Colella [29] using several numerical fluxes.

\begin{tabular}{|c|c|c|c|c|c|c|c|c|}
\hline \multirow[b]{2}{*}{$N$} & \multicolumn{2}{|c|}{$\mathrm{Ch}+\mathrm{SD}$ DWGW } & \multicolumn{2}{|c|}{$\mathrm{Ch}+\mathrm{MD}$ DWGW } & \multicolumn{2}{|c|}{$\mathrm{Ch}+\mathrm{HD}$ DWGW } & \multicolumn{2}{|c|}{$\mathrm{Ch}+\mathrm{LLF}$} \\
\hline & $\left\|\operatorname{err}_{\varrho}\right\|_{M}$ & $\mathrm{EOC}$ & $\left\|\operatorname{err}_{\varrho}\right\|_{M}$ & EOC & $\left\|\operatorname{err}_{\varrho}\right\|_{M}$ & EOC & $\left\|\operatorname{err}_{\varrho}\right\|_{M}$ & $\mathrm{EOC}$ \\
\hline 100 & $7.35 \mathrm{e}-1$ & & $7.09 \mathrm{e}-1$ & & $7.03 \mathrm{e}-1$ & & $7.35 \mathrm{e}-1$ & \\
\hline 200 & $6.60 \mathrm{e}-1$ & 0.16 & $6.33 \mathrm{e}-1$ & 0.16 & $6.24 \mathrm{e}-1$ & 0.17 & $6.59 \mathrm{e}-1$ & 0.16 \\
\hline 400 & $5.32 \mathrm{e}-1$ & 0.31 & $4.92 \mathrm{e}-1$ & 0.36 & $4.89 \mathrm{e}-1$ & 0.35 & $5.32 \mathrm{e}-1$ & 0.31 \\
\hline 800 & $4.33 \mathrm{e}-1$ & 0.30 & $3.92 \mathrm{e}-1$ & 0.33 & $3.91 \mathrm{e}-1$ & 0.32 & $4.33 \mathrm{e}-1$ & 0.30 \\
\hline 1600 & $3.51 \mathrm{e}-1$ & 0.30 & $3.12 \mathrm{e}-1$ & 0.33 & $3.12 \mathrm{e}-1$ & 0.33 & $3.51 \mathrm{e}-1$ & 0.30 \\
\hline 3200 & $2.91 \mathrm{e}-1$ & 0.27 & $2.58 \mathrm{e}-1$ & 0.27 & $2.57 \mathrm{e}-1$ & 0.28 & $2.91 \mathrm{e}-1$ & 0.27 \\
\hline 6400 & $2.38 \mathrm{e}-1$ & 0.29 & $2.06 \mathrm{e}-1$ & 0.32 & $2.07 \mathrm{e}-1$ & 0.31 & $2.38 \mathrm{e}-1$ & 0.29 \\
\hline \multirow[t]{2}{*}{12800} & $1.92 \mathrm{e}-1$ & 0.31 & $*$ & & $1.62 \mathrm{e}-1$ & 0.36 & $1.92 \mathrm{e}-1$ & 0.31 \\
\hline & \multicolumn{2}{|c|}{$\varrho, v, \beta(2)+\mathrm{LLF}$} & \multicolumn{2}{|c|}{$\varrho, v, \frac{1}{p}+\mathrm{LLF}$} & \multicolumn{2}{|c|}{$\varrho, v, p+\mathrm{LLF}$} & \multicolumn{2}{|c|}{$\varrho, v, T(1)+\mathrm{LLF}$} \\
\hline$N$ & $\left\|\operatorname{err}_{\varrho}\right\|_{M}$ & EOC & $\left\|\operatorname{err}_{\varrho}\right\|_{M}$ & EOC & $\left\|\operatorname{err}_{\varrho}\right\|_{M}$ & EOC & $\left\|\operatorname{err}_{\varrho}\right\|_{M}$ & $\mathrm{EOC}$ \\
\hline 100 & $7.34 \mathrm{e}-1$ & & * & & $*$ & & $7.35 \mathrm{e}-1$ & \\
\hline 200 & $6.59 \mathrm{e}-1$ & 0.16 & * & & * & & $6.59 \mathrm{e}-1$ & 0.16 \\
\hline 400 & $5.32 \mathrm{e}-1$ & 0.31 & * & & * & & $5.32 \mathrm{e}-1$ & 0.31 \\
\hline 800 & $4.33 \mathrm{e}-1$ & 0.30 & * & & * & & $4.33 \mathrm{e}-1$ & 0.30 \\
\hline 1600 & $3.51 \mathrm{e}-1$ & 0.30 & * & & * & & $3.51 \mathrm{e}-1$ & 0.30 \\
\hline 3200 & $2.91 \mathrm{e}-1$ & 0.27 & $*$ & & * & & $2.91 \mathrm{e}-1$ & 0.27 \\
\hline 6400 & $2.38 \mathrm{e}-1$ & 0.29 & * & & * & & $2.38 \mathrm{e}-1$ & 0.29 \\
\hline \multirow[t]{2}{*}{12800} & $1.92 \mathrm{e}-1$ & 0.31 & $*$ & & $*$ & & $1.92 \mathrm{e}-1$ & 0.31 \\
\hline & \multicolumn{2}{|c|}{$\varrho, v, T(2)+\mathrm{LLF}$} & \multicolumn{2}{|c|}{$\varrho, V, T(\mathrm{rev})+\mathrm{LLF}$} & \multicolumn{2}{|c|}{ LLF } & \multicolumn{2}{|c|}{ Suliciu } \\
\hline$N$ & $\left\|\operatorname{err}_{\varrho}\right\|_{M}$ & $\mathrm{EOC}$ & $\left\|\operatorname{err}_{\varrho}\right\|_{M}$ & EOC & $\left\|\operatorname{err}_{\varrho}\right\|_{M}$ & EOC & $\left\|\operatorname{err}_{\varrho}\right\|_{M}$ & $\mathrm{EOC}$ \\
\hline 100 & $7.34 \mathrm{e}-1$ & & $7.37 \mathrm{e}-1$ & & $7.42 \mathrm{e}-1$ & & $7.14 \mathrm{e}-1$ & \\
\hline 200 & $6.59 \mathrm{e}-1$ & 0.16 & $6.60 \mathrm{e}-1$ & 0.16 & $6.64 \mathrm{e}-1$ & 0.16 & $6.34 \mathrm{e}-1$ & 0.17 \\
\hline 400 & $5.32 \mathrm{e}-1$ & 0.31 & $5.33 \mathrm{e}-1$ & 0.31 & $5.37 \mathrm{e}-1$ & 0.31 & $4.95 \mathrm{e}-1$ & 0.36 \\
\hline 800 & $4.33 \mathrm{e}-1$ & 0.30 & $4.33 \mathrm{e}-1$ & 0.30 & $4.36 \mathrm{e}-1$ & 0.30 & $3.95 \mathrm{e}-1$ & 0.33 \\
\hline 1600 & $3.51 \mathrm{e}-1$ & 0.30 & $3.51 \mathrm{e}-1$ & 0.30 & $3.53 \mathrm{e}-1$ & 0.30 & $3.15 \mathrm{e}-1$ & 0.33 \\
\hline 3200 & $2.91 \mathrm{e}-1$ & 0.27 & $2.91 \mathrm{e}-1$ & 0.27 & $2.92 \mathrm{e}-1$ & 0.27 & $2.59 \mathrm{e}-1$ & 0.28 \\
\hline 6400 & $2.38 \mathrm{e}-1$ & 0.29 & $2.38 \mathrm{e}-1$ & 0.29 & $2.39 \mathrm{e}-1$ & 0.29 & $2.08 \mathrm{e}-1$ & 0.31 \\
\hline 12800 & $1.92 \mathrm{e}-1$ & 0.31 & $1.92 \mathrm{e}-1$ & 0.31 & $1.93 \mathrm{e}-1$ & 0.31 & $1.64 \mathrm{e}-1$ & 0.35 \\
\hline
\end{tabular}

\subsection{Slowly Moving Contact Discontinuity}

In this section, initial condition of the previous test case is used, but with a non-vanishing initial velocity, resulting in a slowly moving contact discontinuity as described by Toro $[27$, Section 
6.4, Test 5]. The initial condition is given in primitive variables by

$$
\varrho_{0}(x)=1, \quad v_{0}(x)=-19.59745, \quad p_{0}(x)= \begin{cases}1000, & x<\frac{4}{5}, \\ 0.01, & \text { else }\end{cases}
$$

and the conservative variables are again computed via $\varrho v_{0}=\varrho_{0} v_{0}$ and $\varrho e_{0}=\frac{1}{2} \varrho_{0} v_{0}^{2}+\frac{p_{0}}{\gamma-1}$. The solution is computed on the domain $[0,1]$ from $t=0$ until $t=0.012$ using the finite volume methods of section 7.4.

The results are shown in Table 14. As in the previous section 7.7, the fluxes using the pressure in the density flux are unstable. The scalar and LLF dissipation fluxes yield similar errors with some variances across the methods, but the Suliciu solver is superior. In most cases, it is also better than the scalar dissipation (SD).

Table 14: Errors and experimental order of convergence (EOC) for varying number of elements $N$ for the slowly moving contact discontinuity (131) using several numerical fluxes.

\begin{tabular}{|c|c|c|c|c|c|c|c|c|}
\hline \multirow[b]{2}{*}{$N$} & \multicolumn{2}{|c|}{$\mathrm{Ch}+\mathrm{SD}$ DWGW } & \multicolumn{2}{|c|}{ Ch + MD DWGW } & \multicolumn{2}{|c|}{$\mathrm{Ch}+\mathrm{HD}$ DWGW } & \multicolumn{2}{|c|}{$\mathrm{Ch}+\mathrm{LLF}$} \\
\hline & $\left\|\operatorname{err}_{\varrho}\right\|_{M}$ & $\mathrm{EOC}$ & $\left\|\operatorname{err} \varrho_{\varrho}\right\|_{M}$ & $\mathrm{EOC}$ & $\left\|\operatorname{err}_{\varrho}\right\|_{M}$ & EOC & $\left\|\operatorname{err}_{\varrho}\right\|_{M}$ & EOC \\
\hline 100 & $7.84 \mathrm{e}-1$ & & $4.82 \mathrm{e}-1$ & & $6.48 \mathrm{e}-1$ & & $7.85 \mathrm{e}-1$ & \\
\hline 200 & $6.64 \mathrm{e}-1$ & 0.24 & $6.69 \mathrm{e}-1$ & -0.47 & $4.52 \mathrm{e}-1$ & 0.52 & $6.65 \mathrm{e}-1$ & 0.24 \\
\hline 400 & $5.77 \mathrm{e}-1$ & 0.20 & $2.38 \mathrm{e}-1$ & 1.49 & $4.10 \mathrm{e}-1$ & 0.14 & $5.77 \mathrm{e}-1$ & 0.20 \\
\hline 800 & $4.38 \mathrm{e}-1$ & 0.40 & $3.41 \mathrm{e}-1$ & -0.52 & $2.47 \mathrm{e}-1$ & 0.73 & $4.38 \mathrm{e}-1$ & 0.40 \\
\hline 1600 & $3.51 \mathrm{e}-1$ & 0.32 & $2.32 \mathrm{e}-1$ & 0.56 & $1.67 \mathrm{e}-1$ & 0.57 & $3.51 \mathrm{e}-1$ & 0.32 \\
\hline 3200 & $2.97 \mathrm{e}-1$ & 0.24 & $1.02 \mathrm{e}-1$ & 1.18 & $1.40 \mathrm{e}-1$ & 0.25 & $2.97 \mathrm{e}-1$ & 0.24 \\
\hline 6400 & $2.36 \mathrm{e}-1$ & 0.33 & $1.14 \mathrm{e}-1$ & -0.16 & $8.15 \mathrm{e}-2$ & 0.78 & $2.36 \mathrm{e}-1$ & 0.33 \\
\hline \multirow[t]{2}{*}{12800} & $1.99 \mathrm{e}-1$ & 0.25 & $8.59 \mathrm{e}-2$ & 0.41 & $6.57 \mathrm{e}-2$ & 0.31 & $1.99 \mathrm{e}-1$ & 0.25 \\
\hline & \multicolumn{2}{|c|}{$\varrho, v, \beta(2)+\mathrm{LLF}$} & \multicolumn{2}{|c|}{$\varrho, v, \frac{1}{p}+\mathrm{LLF}$} & \multicolumn{2}{|c|}{$\varrho, v, p+\mathrm{LLF}$} & \multicolumn{2}{|c|}{$\varrho, v, T(1)+\mathrm{LLF}$} \\
\hline$N$ & $\left\|\operatorname{err} \varrho_{\varrho}\right\|_{M}$ & $\mathrm{EOC}$ & $\left\|\operatorname{err} \varrho_{\varrho}\right\|_{M}$ & $\mathrm{EOC}$ & $\mid \operatorname{err}_{\varrho} \|_{M}$ & EOC & $\left\|\operatorname{err}_{\varrho}\right\|_{M}$ & EOC \\
\hline 100 & $7.86 \mathrm{e}-1$ & & * & & * & & $7.85 \mathrm{e}-1$ & \\
\hline 200 & $6.65 \mathrm{e}-1$ & 0.24 & * & & * & & $6.65 \mathrm{e}-1$ & 0.24 \\
\hline 400 & $5.76 \mathrm{e}-1$ & 0.21 & * & & * & & $5.77 \mathrm{e}-1$ & 0.20 \\
\hline 800 & $4.38 \mathrm{e}-1$ & 0.40 & * & & * & & $4.38 \mathrm{e}-1$ & 0.40 \\
\hline 1600 & $3.51 \mathrm{e}-1$ & 0.32 & * & & * & & $3.51 \mathrm{e}-1$ & 0.32 \\
\hline 3200 & $2.97 \mathrm{e}-1$ & 0.24 & * & & * & & $2.97 \mathrm{e}-1$ & 0.24 \\
\hline 6400 & $2.36 \mathrm{e}-1$ & 0.33 & * & & * & & $2.36 \mathrm{e}-1$ & 0.33 \\
\hline \multirow[t]{2}{*}{12800} & $1.99 \mathrm{e}-1$ & 0.25 & * & & * & & $1.99 \mathrm{e}-1$ & 0.25 \\
\hline & \multicolumn{2}{|c|}{$\varrho, v, T(2)+\mathrm{LLF}$} & \multicolumn{2}{|c|}{$\varrho, V, T(\mathrm{rev})+\mathrm{LLF}$} & \multicolumn{2}{|c|}{ LLF } & \multicolumn{2}{|c|}{ Suliciu } \\
\hline$N$ & $\left\|\operatorname{err}_{\varrho}\right\|_{M}$ & $\mathrm{EOC}$ & $\left\|\operatorname{err}_{\varrho}\right\|_{M}$ & $\mathrm{EOC}$ & $\left\|\operatorname{err}_{\varrho}\right\|_{M}$ & EOC & $\left\|\operatorname{err}_{\varrho}\right\|_{M}$ & $\mathrm{EOC}$ \\
\hline 100 & $7.86 \mathrm{e}-1$ & & $7.91 \mathrm{e}-1$ & & $7.96 \mathrm{e}-1$ & & $5.08 \mathrm{e}-1$ & \\
\hline 200 & $6.65 \mathrm{e}-1$ & 0.24 & $6.66 \mathrm{e}-1$ & 0.25 & $6.77 \mathrm{e}-1$ & 0.23 & $2.80 \mathrm{e}-1$ & 0.86 \\
\hline 400 & $5.77 \mathrm{e}-1$ & 0.21 & $5.77 \mathrm{e}-1$ & 0.21 & $5.84 \mathrm{e}-1$ & 0.21 & $2.85 \mathrm{e}-1$ & -0.03 \\
\hline 800 & $4.38 \mathrm{e}-1$ & 0.40 & $4.38 \mathrm{e}-1$ & 0.40 & $4.45 \mathrm{e}-1$ & 0.39 & $1.45 \mathrm{e}-1$ & 0.97 \\
\hline 1600 & $3.51 \mathrm{e}-1$ & 0.32 & $3.51 \mathrm{e}-1$ & 0.32 & $3.55 \mathrm{e}-1$ & 0.33 & $9.77 \mathrm{e}-2$ & 0.57 \\
\hline 3200 & $2.97 \mathrm{e}-1$ & 0.24 & $2.97 \mathrm{e}-1$ & 0.24 & $2.99 \mathrm{e}-1$ & 0.24 & $9.03 \mathrm{e}-2$ & 0.11 \\
\hline 6400 & $2.36 \mathrm{e}-1$ & 0.33 & $2.36 \mathrm{e}-1$ & 0.33 & $2.37 \mathrm{e}-1$ & 0.33 & $4.81 \mathrm{e}-2$ & 0.91 \\
\hline 12800 & $1.99 \mathrm{e}-1$ & 0.25 & $1.99 \mathrm{e}-1$ & 0.25 & $1.99 \mathrm{e}-1$ & 0.25 & $3.95 \mathrm{e}-2$ & 0.29 \\
\hline
\end{tabular}

\subsection{Right Half of the Blast Wave Problem of Woodward and Colella}

In this section, the right half of the blast wave problem of Woodward and Colella [29, Section IV.a] as described by Toro [27, Section 4.3.3, Test 4] is considered. In primitive variables, it is given by

$$
\varrho_{0}(x)=1, \quad v_{0}(x)=0, \quad p_{0}(x)= \begin{cases}0.01, & x<\frac{1}{2} \\ 100, & \text { else }\end{cases}
$$


and the conservative variables are again computed via $\varrho v_{0}=\varrho_{0} v_{0}$ and $\varrho e_{0}=\frac{1}{2} \varrho_{0} v_{0}^{2}+\frac{p_{0}}{\gamma-1}$. The solution is computed on the domain $[0,1]$ from $t=0$ until $t=0.035$ using again the finite volume methods described in section 7.4.

The results are shown in Table 15. As before, the fluxes with variables $\varrho, v$ and $p$ or $\frac{1}{p}$ are unstable, and the other fluxes yield similar errors, while the matrix dissipation and Suliciu solver perform a bit better than the others.

Table 15: Errors and experimental order of convergence (EOC) for varying number of elements $N$ for the right half (132) of the blast wave problem of Woodward and Colella [29] using several numerical fluxes.

\begin{tabular}{|c|c|c|c|c|c|c|c|c|}
\hline \multirow[b]{2}{*}{$N$} & \multicolumn{2}{|c|}{$\mathrm{Ch}+\mathrm{SD}$ DWGW } & \multicolumn{2}{|c|}{$\mathrm{Ch}+\mathrm{MD}$ DWGW } & \multicolumn{2}{|c|}{$\mathrm{Ch}+\mathrm{HD}$ DWGW } & \multicolumn{2}{|c|}{$\mathrm{Ch}+\mathrm{LLF}$} \\
\hline & $\left\|\operatorname{err}_{\varrho}\right\|_{M}$ & EOC & $\left\|\operatorname{err}_{\varrho}\right\|_{M}$ & $\mathrm{EOC}$ & $\left\|\operatorname{err}_{\varrho}\right\|_{M}$ & EOC & $\left\|\operatorname{err}_{\varrho}\right\|_{M}$ & EOC \\
\hline 100 & $7.25 \mathrm{e}-1$ & & $6.90 \mathrm{e}-1$ & & $6.89 \mathrm{e}-1$ & & $7.26 \mathrm{e}-1$ & \\
\hline 200 & $6.45 \mathrm{e}-1$ & 0.17 & $6.11 \mathrm{e}-1$ & 0.18 & $6.05 \mathrm{e}-1$ & 0.19 & $6.45 \mathrm{e}-1$ & 0.17 \\
\hline 400 & $5.26 \mathrm{e}-1$ & 0.29 & $4.91 \mathrm{e}-1$ & 0.32 & $4.87 \mathrm{e}-1$ & 0.31 & $5.26 \mathrm{e}-1$ & 0.30 \\
\hline 800 & $4.38 \mathrm{e}-1$ & 0.26 & $4.02 \mathrm{e}-1$ & 0.29 & $3.99 \mathrm{e}-1$ & 0.29 & $4.38 \mathrm{e}-1$ & 0.26 \\
\hline 1600 & $3.58 \mathrm{e}-1$ & 0.29 & $3.24 \mathrm{e}-1$ & 0.31 & $3.22 \mathrm{e}-1$ & 0.31 & $3.57 \mathrm{e}-1$ & 0.29 \\
\hline 3200 & $2.86 \mathrm{e}-1$ & 0.32 & $2.53 \mathrm{e}-1$ & 0.36 & $2.53 \mathrm{e}-1$ & 0.35 & $2.86 \mathrm{e}-1$ & 0.32 \\
\hline 6400 & $2.33 \mathrm{e}-1$ & 0.29 & $2.02 \mathrm{e}-1$ & 0.33 & $2.03 \mathrm{e}-1$ & 0.32 & $2.33 \mathrm{e}-1$ & 0.29 \\
\hline \multirow[t]{2}{*}{12800} & $1.88 \mathrm{e}-1$ & 0.31 & $1.64 \mathrm{e}-1$ & 0.30 & $1.59 \mathrm{e}-1$ & 0.35 & $1.88 \mathrm{e}-1$ & 0.31 \\
\hline & \multicolumn{2}{|c|}{$\varrho, v, \beta(2)+\mathrm{LLF}$} & \multicolumn{2}{|c|}{$\varrho, v, \frac{1}{p}+\mathrm{LLF}$} & \multicolumn{2}{|c|}{$\varrho, v, p+\mathrm{LLF}$} & \multicolumn{2}{|c|}{$\varrho, v, T(1)+\mathrm{LLF}$} \\
\hline$N$ & $\left\|\operatorname{err}_{\varrho}\right\|_{M}$ & $\mathrm{EOC}$ & $\left\|\operatorname{err}_{\varrho}\right\|_{M}$ & $\mathrm{EOC}$ & $\mid \operatorname{err}_{\varrho} \|_{M}$ & EOC & $\left\|\operatorname{err}_{\varrho}\right\|_{M}$ & EOC \\
\hline 100 & $7.24 \mathrm{e}-1$ & & * & & * & & $7.26 \mathrm{e}-1$ & \\
\hline 200 & $6.44 \mathrm{e}-1$ & 0.17 & * & & * & & $6.45 \mathrm{e}-1$ & 0.17 \\
\hline 400 & $5.25 \mathrm{e}-1$ & 0.29 & * & & * & & $5.26 \mathrm{e}-1$ & 0.30 \\
\hline 800 & $4.38 \mathrm{e}-1$ & 0.26 & * & & * & & $4.38 \mathrm{e}-1$ & 0.26 \\
\hline 1600 & $3.57 \mathrm{e}-1$ & 0.29 & * & & * & & $3.57 \mathrm{e}-1$ & 0.29 \\
\hline 3200 & $2.86 \mathrm{e}-1$ & 0.32 & * & & * & & $2.86 \mathrm{e}-1$ & 0.32 \\
\hline 6400 & $2.33 \mathrm{e}-1$ & 0.29 & * & & * & & $2.33 \mathrm{e}-1$ & 0.29 \\
\hline \multirow[t]{2}{*}{12800} & $1.88 \mathrm{e}-1$ & 0.31 & * & & * & & $1.88 \mathrm{e}-1$ & 0.31 \\
\hline & \multicolumn{2}{|c|}{$\varrho, v, T(2)+\mathrm{LLF}$} & \multicolumn{2}{|c|}{$\varrho, V, T$ (rev) + LLF } & \multicolumn{2}{|c|}{ LLF } & \multicolumn{2}{|c|}{ Suliciu } \\
\hline$N$ & $\left\|\operatorname{err}_{\varrho}\right\|_{M}$ & $\mathrm{EOC}$ & $\left\|\operatorname{err}_{\varrho}\right\|_{M}$ & EOC & $\left\|\operatorname{err}_{\varrho}\right\|_{M}$ & EOC & $\left\|\operatorname{err}_{\varrho}\right\|_{M}$ & EOC \\
\hline 100 & $7.25 \mathrm{e}-1$ & & $7.28 \mathrm{e}-1$ & & $7.35 \mathrm{e}-1$ & & $7.00 \mathrm{e}-1$ & \\
\hline 200 & $6.44 \mathrm{e}-1$ & 0.17 & $6.46 \mathrm{e}-1$ & 0.17 & $6.52 \mathrm{e}-1$ & 0.17 & $6.16 \mathrm{e}-1$ & 0.19 \\
\hline 400 & $5.25 \mathrm{e}-1$ & 0.29 & $5.26 \mathrm{e}-1$ & 0.30 & $5.30 \mathrm{e}-1$ & 0.30 & $4.92 \mathrm{e}-1$ & 0.32 \\
\hline 800 & $4.38 \mathrm{e}-1$ & 0.26 & $4.38 \mathrm{e}-1$ & 0.26 & $4.41 \mathrm{e}-1$ & 0.27 & $4.03 \mathrm{e}-1$ & 0.29 \\
\hline 1600 & $3.57 \mathrm{e}-1$ & 0.29 & $3.58 \mathrm{e}-1$ & 0.29 & $3.59 \mathrm{e}-1$ & 0.30 & $3.24 \mathrm{e}-1$ & 0.31 \\
\hline 3200 & $2.86 \mathrm{e}-1$ & 0.32 & $2.86 \mathrm{e}-1$ & 0.32 & $2.87 \mathrm{e}-1$ & 0.32 & $2.54 \mathrm{e}-1$ & 0.35 \\
\hline 6400 & $2.33 \mathrm{e}-1$ & 0.29 & $2.33 \mathrm{e}-1$ & 0.29 & $2.34 \mathrm{e}-1$ & 0.30 & $2.04 \mathrm{e}-1$ & 0.32 \\
\hline 12800 & $1.88 \mathrm{e}-1$ & 0.31 & $1.88 \mathrm{e}-1$ & 0.31 & $1.89 \mathrm{e}-1$ & 0.31 & $1.60 \mathrm{e}-1$ & 0.35 \\
\hline
\end{tabular}

\subsection{Left Half of the Blast Wave Problem of Derigs, Winters, Gassner and Walch}

In this section, the left half of the blast wave problem of Derigs, Winters, Gassner, and Walch $[6$, Section 6] is considered. In primitive variables, it is given by

$$
\varrho_{0}(x)=1, \quad v_{0}(x)=10, \quad p_{0}(x)= \begin{cases}1, & x<-\frac{1}{10}, \\ 10^{-6}, & \text { else } .\end{cases}
$$

Here, $\gamma=\frac{5}{3}$ is used. The solution is computed on the domain $[-1,1]$ from $t=0$ until $t=$ $5.0 \times 10^{-2}$ using the finite volume methods of section 7.4.

The results are shown in Table 16. Designed as a test case to crash the flux of Roe [22], the fluxes containing pressure influence in the density flux are unstable. However, there is nearly 
no variance across the other fluxes that remain stable, since the problem needs a very high resolution to capture the solution.

Contrary to the results of Derigs, Winters, Gassner, and Walch [6] for the MHD equations, the simple LLF dissipation is enough to stabilise the solution for the Euler equations in this case and their specially designed dissipation operator does not show any improvement over the LLF dissipation.

Table 16: Errors and experimental order of convergence (EOC) for varying number of elements $N$ for the left half (133) of the blast wave problem of Derigs, Winters, Gassner, and Walch [6] using several numerical fluxes.

\begin{tabular}{|c|c|c|c|c|c|c|c|c|}
\hline \multirow[b]{2}{*}{$N$} & \multicolumn{2}{|c|}{$\mathrm{Ch}+\mathrm{SD}$ DWGW } & \multicolumn{2}{|c|}{$\mathrm{Ch}+\mathrm{MD}$ DWGW } & \multicolumn{2}{|c|}{$\mathrm{Ch}+\mathrm{HD}$ DWGW } & \multicolumn{2}{|c|}{$\mathrm{Ch}+\mathrm{LLF}$} \\
\hline & $\left\|\operatorname{err}_{\varrho}\right\|_{M}$ & EOC & $\left\|\operatorname{err}_{\varrho}\right\|_{M}$ & $\mathrm{EOC}$ & $\left\|\operatorname{err}_{\varrho}\right\|_{M}$ & EOC & $\left\|\operatorname{err}_{\varrho}\right\|_{M}$ & $\mathrm{EOC}$ \\
\hline 100 & $4.32 \mathrm{e}-1$ & & $4.33 \mathrm{e}-1$ & & $4.33 \mathrm{e}-1$ & & $4.33 \mathrm{e}-1$ & \\
\hline 200 & $3.13 \mathrm{e}-1$ & 0.47 & $3.13 \mathrm{e}-1$ & 0.47 & $3.13 \mathrm{e}-1$ & 0.47 & $3.13 \mathrm{e}-1$ & 0.47 \\
\hline 400 & $3.08 \mathrm{e}-1$ & 0.02 & $3.07 \mathrm{e}-1$ & 0.02 & $3.08 \mathrm{e}-1$ & 0.02 & $3.08 \mathrm{e}-1$ & 0.02 \\
\hline 800 & $2.64 \mathrm{e}-1$ & 0.22 & $2.63 \mathrm{e}-1$ & 0.23 & $2.63 \mathrm{e}-1$ & 0.23 & $2.64 \mathrm{e}-1$ & 0.22 \\
\hline 1600 & $2.83 \mathrm{e}-1$ & -0.10 & $2.82 \mathrm{e}-1$ & -0.10 & $2.82 \mathrm{e}-1$ & -0.10 & $2.84 \mathrm{e}-1$ & -0.10 \\
\hline 3200 & $2.60 \mathrm{e}-1$ & 0.13 & $2.58 \mathrm{e}-1$ & 0.13 & $2.58 \mathrm{e}-1$ & 0.13 & $2.60 \mathrm{e}-1$ & 0.13 \\
\hline 6400 & $2.24 \mathrm{e}-1$ & 0.21 & $2.23 \mathrm{e}-1$ & 0.21 & $2.23 \mathrm{e}-1$ & 0.21 & $2.24 \mathrm{e}-1$ & 0.21 \\
\hline \multirow{2}{*}{12800} & $1.78 \mathrm{e}-1$ & 0.33 & $1.77 \mathrm{e}-1$ & 0.33 & $1.77 \mathrm{e}-1$ & 0.33 & $1.78 \mathrm{e}-1$ & 0.33 \\
\hline & \multicolumn{2}{|c|}{$\varrho, v, \beta(2)+\mathrm{LLF}$} & \multicolumn{2}{|c|}{$\varrho, v, \frac{1}{p}+\mathrm{LLF}$} & \multicolumn{2}{|c|}{$\varrho, v, p+\mathrm{LLF}$} & \multicolumn{2}{|c|}{$\varrho, v, T(1)+\mathrm{LLF}$} \\
\hline$N$ & $\left\|\operatorname{err}_{\varrho}\right\|_{M}$ & EOC & $\left\|\operatorname{err}_{\varrho}\right\|_{M}$ & EOC & $\left\|\operatorname{err}_{\varrho}\right\|_{M}$ & EOC & $\left\|\operatorname{err}_{\varrho}\right\|_{M}$ & EOC \\
\hline 100 & $4.33 \mathrm{e}-1$ & & * & & * & & $4.33 \mathrm{e}-1$ & \\
\hline 200 & $3.13 \mathrm{e}-1$ & 0.47 & * & & * & & $3.13 \mathrm{e}-1$ & 0.47 \\
\hline 400 & $3.08 \mathrm{e}-1$ & 0.02 & * & & * & & $3.08 \mathrm{e}-1$ & 0.02 \\
\hline 800 & $2.64 \mathrm{e}-1$ & 0.22 & * & & * & & $2.64 \mathrm{e}-1$ & 0.22 \\
\hline 1600 & $2.83 \mathrm{e}-1$ & -0.10 & * & & * & & $2.84 \mathrm{e}-1$ & -0.10 \\
\hline 3200 & $2.60 \mathrm{e}-1$ & 0.13 & * & & * & & $2.60 \mathrm{e}-1$ & 0.13 \\
\hline 6400 & $2.24 \mathrm{e}-1$ & 0.21 & * & & * & & $2.24 \mathrm{e}-1$ & 0.21 \\
\hline \multirow[t]{2}{*}{12800} & $1.78 \mathrm{e}-1$ & 0.33 & $*$ & & * & & $1.78 \mathrm{e}-1$ & 0.33 \\
\hline & \multicolumn{2}{|c|}{$\varrho, v, T(2)+\mathrm{LLF}$} & \multicolumn{2}{|c|}{$\varrho, V, T$ (rev) + LLF } & \multicolumn{2}{|c|}{ LLF } & \multicolumn{2}{|c|}{ Suliciu } \\
\hline$N$ & $\left\|\operatorname{err}_{\varrho}\right\|_{M}$ & EOC & $\left\|\operatorname{err}_{\varrho}\right\|_{M}$ & EOC & $\left\|\operatorname{err}_{\varrho}\right\|_{M}$ & EOC & $\left\|\operatorname{err}_{\varrho}\right\|_{M}$ & $\mathrm{EOC}$ \\
\hline 100 & $4.33 \mathrm{e}-1$ & & $4.33 \mathrm{e}-1$ & & $4.33 \mathrm{e}-1$ & & $4.33 \mathrm{e}-1$ & \\
\hline 200 & $3.13 \mathrm{e}-1$ & 0.47 & $3.13 \mathrm{e}-1$ & 0.47 & $3.13 \mathrm{e}-1$ & 0.47 & $3.13 \mathrm{e}-1$ & 0.47 \\
\hline 400 & $3.08 \mathrm{e}-1$ & 0.02 & $3.08 \mathrm{e}-1$ & 0.02 & $3.09 \mathrm{e}-1$ & 0.02 & $3.08 \mathrm{e}-1$ & 0.02 \\
\hline 800 & $2.64 \mathrm{e}-1$ & 0.22 & $2.64 \mathrm{e}-1$ & 0.22 & $2.64 \mathrm{e}-1$ & 0.22 & $2.63 \mathrm{e}-1$ & 0.23 \\
\hline 1600 & $2.83 \mathrm{e}-1$ & -0.10 & $2.84 \mathrm{e}-1$ & -0.10 & $2.84 \mathrm{e}-1$ & -0.10 & $2.83 \mathrm{e}-1$ & -0.10 \\
\hline 3200 & $2.60 \mathrm{e}-1$ & 0.13 & $2.60 \mathrm{e}-1$ & 0.13 & $2.60 \mathrm{e}-1$ & 0.13 & $2.59 \mathrm{e}-1$ & 0.13 \\
\hline 6400 & $2.24 \mathrm{e}-1$ & 0.21 & $2.24 \mathrm{e}-1$ & 0.21 & $2.24 \mathrm{e}-1$ & 0.21 & $2.23 \mathrm{e}-1$ & 0.22 \\
\hline 12800 & $1.78 \mathrm{e}-1$ & 0.33 & $1.78 \mathrm{e}-1$ & 0.33 & $1.78 \mathrm{e}-1$ & 0.33 & $1.77 \mathrm{e}-1$ & 0.33 \\
\hline
\end{tabular}

\subsection{Right Half of the Blast Wave Problem of Derigs, Winters, Gassner and Walch}

In this section, the right half of the blast wave problem of Derigs, Winters, Gassner, and Walch $[6$, Section 6$]$ is considered. In primitive variables, it is given by

$$
\varrho_{0}(x)=1, \quad v_{0}(x)=10, \quad p_{0}(x)= \begin{cases}10^{-6}, & x<\frac{1}{10}, \\ 1, & \text { else }\end{cases}
$$

Here, $\gamma=\frac{5}{3}$ is used. The solution is computed on the domain $[-1,1]$ from $t=0$ until $t=$ $5.0 \times 10^{-2}$ using again the same finite volume methods as before. 
The results are shown in Table 17. The results are similar to the left half of this problem in section 7.10: The pressure influence in the density flux results in unstable schemes while all other methods yield similar errors and are stable.

Table 17: Errors and experimental order of convergence (EOC) for varying number of elements $N$ for the right half (134) of the blast wave problem of Derigs, Winters, Gassner, and Walch [6] using several numerical fluxes.

\begin{tabular}{|c|c|c|c|c|c|c|c|c|}
\hline \multirow[b]{2}{*}{$N$} & \multicolumn{2}{|c|}{$\mathrm{Ch}+\mathrm{SD}$ DWGW } & \multicolumn{2}{|c|}{$\mathrm{Ch}+\mathrm{MD}$ DWGW } & \multicolumn{2}{|c|}{$\mathrm{Ch}+\mathrm{HD}$ DWGW } & \multicolumn{2}{|c|}{$\mathrm{Ch}+\mathrm{LLF}$} \\
\hline & $\left\|\operatorname{err}_{\varrho}\right\|_{M}$ & EOC & $\left\|\operatorname{err}_{\varrho}\right\|_{M}$ & EOC & $\left\|\operatorname{err}_{\varrho}\right\|_{M}$ & EOC & $\left\|\operatorname{err}_{\varrho}\right\|_{M}$ & $\mathrm{EOC}$ \\
\hline 100 & $4.33 \mathrm{e}-1$ & & $4.32 \mathrm{e}-1$ & & $4.33 \mathrm{e}-1$ & & $4.33 \mathrm{e}-1$ & \\
\hline 200 & $3.13 \mathrm{e}-1$ & 0.47 & $3.13 \mathrm{e}-1$ & 0.47 & $3.13 \mathrm{e}-1$ & 0.47 & $3.13 \mathrm{e}-1$ & 0.47 \\
\hline 400 & $3.09 \mathrm{e}-1$ & 0.02 & $3.07 \mathrm{e}-1$ & 0.03 & $3.08 \mathrm{e}-1$ & 0.02 & $3.09 \mathrm{e}-1$ & 0.02 \\
\hline 800 & $2.64 \mathrm{e}-1$ & 0.22 & $2.63 \mathrm{e}-1$ & 0.23 & $2.63 \mathrm{e}-1$ & 0.23 & $2.64 \mathrm{e}-1$ & 0.22 \\
\hline 1600 & $2.83 \mathrm{e}-1$ & -0.10 & $2.80 \mathrm{e}-1$ & -0.09 & $2.80 \mathrm{e}-1$ & -0.09 & $2.83 \mathrm{e}-1$ & -0.10 \\
\hline 3200 & $2.58 \mathrm{e}-1$ & 0.13 & $2.54 \mathrm{e}-1$ & 0.14 & $2.55 \mathrm{e}-1$ & 0.14 & $2.58 \mathrm{e}-1$ & 0.13 \\
\hline 6400 & $2.23 \mathrm{e}-1$ & 0.21 & $2.18 \mathrm{e}-1$ & 0.22 & $2.18 \mathrm{e}-1$ & 0.22 & $2.23 \mathrm{e}-1$ & 0.21 \\
\hline \multirow[t]{2}{*}{12800} & $1.79 \mathrm{e}-1$ & 0.32 & $1.73 \mathrm{e}-1$ & 0.33 & $1.74 \mathrm{e}-1$ & 0.33 & $1.79 \mathrm{e}-1$ & 0.32 \\
\hline & \multicolumn{2}{|c|}{$\varrho, v, \beta(2)+\mathrm{LLF}$} & \multicolumn{2}{|c|}{$\varrho, v, \frac{1}{p}+\mathrm{LLF}$} & \multicolumn{2}{|c|}{$\varrho, v, p+\mathrm{LLF}$} & \multicolumn{2}{|c|}{$\varrho, v, T(1)+\mathrm{LLF}$} \\
\hline$N$ & $\left\|\operatorname{err}_{\varrho}\right\|_{M}$ & $\mathrm{EOC}$ & $\left\|\operatorname{err}_{\varrho}\right\|_{M}$ & EOC & $\mid \operatorname{err}_{\varrho} \|_{M}$ & EOC & $\left\|\operatorname{err}_{\varrho}\right\|_{M}$ & $\mathrm{EOC}$ \\
\hline 100 & $4.33 \mathrm{e}-1$ & & * & & $*$ & & $4.33 \mathrm{e}-1$ & \\
\hline 200 & $3.14 \mathrm{e}-1$ & 0.47 & * & & * & & $3.13 \mathrm{e}-1$ & 0.47 \\
\hline 400 & $3.09 \mathrm{e}-1$ & 0.02 & * & & * & & $3.09 \mathrm{e}-1$ & 0.02 \\
\hline 800 & $2.64 \mathrm{e}-1$ & 0.22 & * & & * & & $2.64 \mathrm{e}-1$ & 0.22 \\
\hline 1600 & $2.83 \mathrm{e}-1$ & -0.10 & * & & * & & $2.83 \mathrm{e}-1$ & -0.10 \\
\hline 3200 & $2.59 \mathrm{e}-1$ & 0.13 & * & & * & & $2.58 \mathrm{e}-1$ & 0.13 \\
\hline 6400 & $2.23 \mathrm{e}-1$ & 0.21 & * & & * & & $2.23 \mathrm{e}-1$ & 0.21 \\
\hline \multirow[t]{2}{*}{12800} & $1.79 \mathrm{e}-1$ & 0.32 & * & & * & & $1.79 \mathrm{e}-1$ & 0.32 \\
\hline & \multicolumn{2}{|c|}{$\varrho, v, T(2)+\mathrm{LLF}$} & \multicolumn{2}{|c|}{$\varrho, V, T$ (rev) $+\mathrm{LLF}$} & \multicolumn{2}{|c|}{ LLF } & \multicolumn{2}{|c|}{ Suliciu } \\
\hline$N$ & $\left\|\operatorname{err}_{\varrho}\right\|_{M}$ & EOC & $\left\|\operatorname{err}_{\varrho}\right\|_{M}$ & EOC & $\left\|\operatorname{err}_{\varrho}\right\|_{M}$ & EOC & $\left\|\operatorname{err}_{\varrho}\right\|_{M}$ & EOC \\
\hline 100 & $4.33 \mathrm{e}-1$ & & $4.33 \mathrm{e}-1$ & & $4.33 \mathrm{e}-1$ & & $4.32 \mathrm{e}-1$ & \\
\hline 200 & $3.14 \mathrm{e}-1$ & 0.47 & $3.14 \mathrm{e}-1$ & 0.47 & $3.13 \mathrm{e}-1$ & 0.47 & $3.13 \mathrm{e}-1$ & 0.47 \\
\hline 400 & $3.09 \mathrm{e}-1$ & 0.02 & $3.09 \mathrm{e}-1$ & 0.02 & $3.08 \mathrm{e}-1$ & 0.02 & $3.07 \mathrm{e}-1$ & 0.03 \\
\hline 800 & $2.64 \mathrm{e}-1$ & 0.22 & $2.64 \mathrm{e}-1$ & 0.22 & $2.64 \mathrm{e}-1$ & 0.23 & $2.62 \mathrm{e}-1$ & 0.23 \\
\hline 1600 & $2.83 \mathrm{e}-1$ & -0.10 & $2.83 \mathrm{e}-1$ & -0.10 & $2.83 \mathrm{e}-1$ & -0.10 & $2.80 \mathrm{e}-1$ & -0.09 \\
\hline 3200 & $2.59 \mathrm{e}-1$ & 0.13 & $2.58 \mathrm{e}-1$ & 0.13 & $2.58 \mathrm{e}-1$ & 0.13 & $2.54 \mathrm{e}-1$ & 0.14 \\
\hline 6400 & $2.23 \mathrm{e}-1$ & 0.21 & $2.23 \mathrm{e}-1$ & 0.21 & $2.23 \mathrm{e}-1$ & 0.21 & $2.18 \mathrm{e}-1$ & 0.22 \\
\hline 12800 & $1.79 \mathrm{e}-1$ & 0.32 & $1.79 \mathrm{e}-1$ & 0.32 & $1.79 \mathrm{e}-1$ & 0.32 & $1.73 \mathrm{e}-1$ & 0.33 \\
\hline
\end{tabular}

\subsection{Another Blast Wave Problem}

In this section, another blast wave problem is considered. In primitive variables, it is given by

$$
\varrho_{0}(x)=\frac{1}{10}, \quad v_{0}(x)=10, \quad p_{0}(x)= \begin{cases}10^{-12}, & x<-\frac{1}{2}, \\ 10^{-3}, & \text { else. }\end{cases}
$$

Here, $\gamma=\frac{5}{3}$ is used. The solution is computed on the domain $[-1,1]$ from $t=0$ until $t=0.1$ using the same set of FV methods as in the previous test cases.

To the author's knowledge, this test problem has not been used before, and is designed to show the importance of positivity preserving for the pressure. As can be seen in the results shown in Table 18, the new scalar and matrix dissipation operators of Derigs, Winters, Gassner, and Walch [6] and Winters, Derigs, Gassner, and Walch [28] are not stable for this problem. Indeed, they result in negative pressures. However, the simple LLF dissipation that has been reported to be less stable than these dissipation operators by Derigs, Winters, Gassner, and Walch [6] for the MHD equations remains stable in this test case. 
Of course, the fluxes containing an influence of the pressure in the density flux are unstable. The remaining fluxes (with LLF dissipation and Suliciu) are all stable and result in the same error (up to two digits of precision).

As another example demonstrating the positivity preserving issue for the pressure, explicit Euler FV steps (110) using the entropy conservative flux (52) of Chandrashekar [3] with scalar and matrix dissipation operators by Derigs, Winters, Gassner, and Walch [6] and Winters, Derigs, Gassner, and Walch [28] as well as LLF dissipation, respectively, have been performed with the states

$$
\left(\begin{array}{l}
\varrho \\
v \\
p
\end{array}\right)_{i-1}=\left(\begin{array}{l}
\varrho \\
v \\
p
\end{array}\right)_{i}=\left(\begin{array}{c}
0.1 \\
10 \\
1.0 \times 10^{-12}
\end{array}\right), \quad\left(\begin{array}{l}
\varrho \\
v \\
p
\end{array}\right)_{i+1}=\left(\begin{array}{c}
10 \\
10 \\
1.0 \times 10^{-6}
\end{array}\right) .
$$

As can be seen in Figure 1, the pressure becomes negative for both the scalar $\left[\frac{\Delta t}{\Delta x} \lesssim 0.3 \times 10^{-12}\right]$ and the matrix dissipation $\left[\frac{\Delta t}{\Delta x} \lesssim 0.1 \times 10^{-12}\right]$ operator, while the characteristic speeds on the left $(i-1, i)$ and right $(i+1)$ hand side are $v=10, c_{i}=\sqrt{\gamma \frac{\underline{p}_{i}}{\varrho_{i}}}=\sqrt{10^{-11} \gamma}$, and $c_{i+1}=\sqrt{10^{-13} \gamma}$. Thus, there does not seem to be a reasonable CFL condition for these two fluxes and for this initial condition. Contrary, the LLF dissipation operator results in a positive pressure.

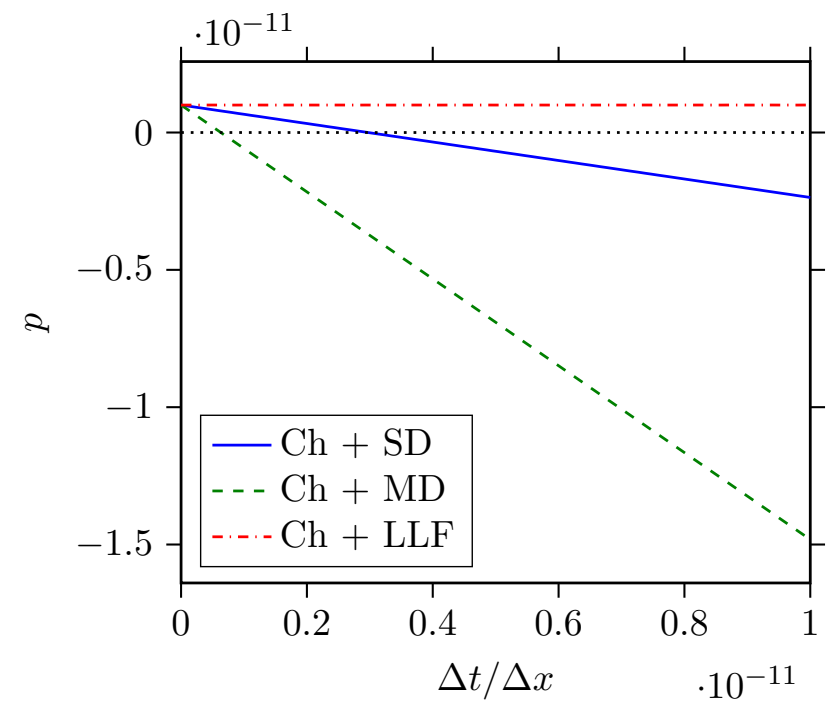

Figure 1: Pressure depending on the step size ration $\frac{\Delta t}{\Delta x}$ for one FV step (110) using a scalar (solid), matrix (dashed), and LLF (dash-dotted) dissipation operator for the initial condition (136).

\subsection{Summary of the Numerical Results}

There are three main results of these numerical tests. Firstly, none of the entropy conservative fluxes not including an influence of the pressure in the density flux seems to be clearly superior to the others.

Secondly, the entropy conservative volume fluxes result in schemes that are more robust for discontinuous solutions than the schemes using the other fluxes. Nevertheless, coupling entropy conservative volume fluxes with dissipative surface fluxes is not sufficient for strong shocks. Thus, these results should be considered carefully, since no additional shock capturing mechanisms - which will be needed in practice - have been used.

Finally, enhancing the entropy conservative fluxes not using the pressure in the density flux by a local Lax-Friedrichs type dissipation $-\frac{\lambda}{2} \llbracket u \rrbracket$ is more robust concerning positivity of the pressure than dissipation operators that have been developed for the MHD equations and transferred to the Euler equations. 
Table 18: Errors and experimental order of convergence (EOC) for varying number of elements $N$ for the blast wave (135) using several numerical fluxes.

\begin{tabular}{|c|c|c|c|c|c|c|c|c|}
\hline \multirow[b]{2}{*}{$N$} & \multicolumn{2}{|c|}{$\mathrm{Ch}+\mathrm{SD}$ DWGW } & \multicolumn{2}{|c|}{$\mathrm{Ch}+\mathrm{MD} \mathrm{DWGW}$} & \multicolumn{2}{|c|}{$\mathrm{Ch}+\mathrm{HD} \mathrm{DWGW}$} & \multicolumn{2}{|c|}{$\mathrm{Ch}+\mathrm{LLF}$} \\
\hline & $\left\|\operatorname{err}_{\varrho}\right\|_{M}$ & EOC & $\left\|\operatorname{err}_{\varrho}\right\|_{M}$ & EOC & $\left\|\operatorname{err}_{\varrho}\right\|_{M}$ & EOC & $\left\|\operatorname{err}_{\varrho}\right\|_{M}$ & EOC \\
\hline 100 & * & & * & & * & & $1.73 \mathrm{e}-3$ & \\
\hline 200 & $*$ & & $*$ & & $*$ & & $5.82 \mathrm{e}-3$ & -1.75 \\
\hline 400 & $*$ & & * & & $*$ & & $4.66 \mathrm{e}-3$ & 0.32 \\
\hline 800 & $*$ & & * & & $*$ & & $2.54 \mathrm{e}-2$ & -2.45 \\
\hline 1600 & * & & * & & * & & $1.83 \mathrm{e}-2$ & 0.47 \\
\hline 3200 & * & & * & & * & & $1.83 \mathrm{e}-2$ & 0.00 \\
\hline 6400 & * & & * & & $*$ & & $1.82 \mathrm{e}-2$ & 0.01 \\
\hline 12800 & * & & * & & $*$ & & $1.78 \mathrm{e}-2$ & 0.04 \\
\hline \multirow[b]{2}{*}{$N$} & \multicolumn{2}{|c|}{$\varrho, v, \beta(2)+\mathrm{LLF}$} & \multicolumn{2}{|c|}{$\varrho, v, \frac{1}{p}+\mathrm{LLF}$} & \multicolumn{2}{|c|}{$\varrho, v, p+\mathrm{LLF}$} & \multicolumn{2}{|c|}{$\varrho, v, T(1)+\mathrm{LLF}$} \\
\hline & $\left\|\operatorname{err}_{\varrho}\right\|_{M}$ & EOC & $\left\|\operatorname{err}_{\varrho}\right\|_{M}$ & EOC & $\mid \operatorname{err}_{\varrho} \|_{M}$ & EOC & $\left\|\operatorname{err}_{\varrho}\right\|_{M}$ & EOC \\
\hline 100 & \multicolumn{2}{|l|}{$1.73 \mathrm{e}-3$} & \multicolumn{2}{|l|}{ * } & \multicolumn{2}{|l|}{$*$} & \multicolumn{2}{|l|}{$1.73 \mathrm{e}-3$} \\
\hline 200 & \multirow{2}{*}{$5.82 \mathrm{e}-3$} & -1.75 & $*$ & & \multicolumn{2}{|l|}{$*$} & $5.82 \mathrm{e}-3$ & -1.75 \\
\hline 400 & & 0.32 & $*$ & & \multicolumn{2}{|l|}{ * } & $4.66 \mathrm{e}-3$ & 0.32 \\
\hline 800 & $2.54 \mathrm{e}-2$ & -2.45 & * & & \multicolumn{2}{|l|}{$*$} & $2.54 \mathrm{e}-2$ & -2.45 \\
\hline 1600 & $1.83 \mathrm{e}-2$ & 0.47 & * & & \multicolumn{2}{|l|}{$*$} & $1.83 \mathrm{e}-2$ & 0.47 \\
\hline 3200 & $1.83 \mathrm{e}-2$ & 0.00 & $*$ & & \multicolumn{2}{|l|}{ * } & $1.83 \mathrm{e}-2$ & 0.00 \\
\hline 6400 & $1.82 \mathrm{e}-2$ & 0.01 & * & & \multicolumn{2}{|c|}{$*$} & $1.82 \mathrm{e}-2$ & 0.01 \\
\hline 12800 & $1.78 \mathrm{e}-2$ & 0.04 & * & & \multicolumn{2}{|l|}{ * } & $1.78 \mathrm{e}-2$ & 0.04 \\
\hline & \multicolumn{2}{|c|}{$\varrho, v, T(2)+\mathrm{LLF}$} & \multicolumn{2}{|c|}{$\varrho, V, T(\mathrm{rev})+\mathrm{LLF}$} & \multicolumn{2}{|c|}{ LLF } & Suli & \\
\hline$N$ & $\left\|\operatorname{err}_{\varrho}\right\|_{M}$ & EOC & $\left\|\operatorname{err}_{\varrho}\right\|_{M}$ & EOC & $\left\|\operatorname{err}_{\varrho}\right\|_{M}$ & EOC & $\left\|\operatorname{err}_{\varrho}\right\|_{M}$ & EOC \\
\hline 100 & $1.73 \mathrm{e}-3$ & & $1.73 \mathrm{e}-3$ & & $1.73 \mathrm{e}-3$ & & $1.73 \mathrm{e}-3$ & \\
\hline 200 & $5.82 \mathrm{e}-3$ & -1.75 & $5.82 \mathrm{e}-3$ & -1.75 & $5.82 \mathrm{e}-3$ & -1.75 & $5.82 \mathrm{e}-3$ & -1.75 \\
\hline 400 & $4.66 \mathrm{e}-3$ & 0.32 & $4.66 \mathrm{e}-3$ & 0.32 & $4.66 \mathrm{e}-3$ & 0.32 & $4.66 \mathrm{e}-3$ & 0.32 \\
\hline 800 & $2.54 \mathrm{e}-2$ & -2.45 & $2.54 \mathrm{e}-2$ & -2.45 & $2.54 \mathrm{e}-2$ & -2.45 & $2.54 \mathrm{e}-2$ & -2.45 \\
\hline 1600 & $1.83 \mathrm{e}-2$ & 0.47 & $1.83 \mathrm{e}-2$ & 0.47 & $1.83 \mathrm{e}-2$ & 0.47 & $1.83 \mathrm{e}-2$ & 0.47 \\
\hline 3200 & $1.83 \mathrm{e}-2$ & 0.00 & $1.83 \mathrm{e}-2$ & 0.00 & $1.83 \mathrm{e}-2$ & 0.00 & $1.83 \mathrm{e}-2$ & 0.00 \\
\hline 6400 & $1.82 \mathrm{e}-2$ & 0.01 & $1.82 \mathrm{e}-2$ & 0.01 & $1.82 \mathrm{e}-2$ & 0.01 & $1.82 \mathrm{e}-2$ & 0.01 \\
\hline 12800 & $1.78 \mathrm{e}-2$ & 0.04 & $1.78 \mathrm{e}-2$ & 0.04 & $1.78 \mathrm{e}-2$ & 0.04 & $1.78 \mathrm{e}-2$ & 0.04 \\
\hline
\end{tabular}




\section{Summary and Conclusions}

After formulating a general procedure to develop affordable entropy conservative fluxes, several new numerical fluxes for the Euler equations have been developed in sections 4 and 5 and compared with existing ones in two kinds of application.

Firstly, the entropy conservative fluxes can be used as building blocks of entropy stable highorder schemes using the flux differencing form of Fisher and Carpenter [10]. In section 3, the high order of accuracy of the flux differencing form has been proven for consistent and symmetric numerical fluxes, extending the known theory of [10]. Moreover, entropy conservation and stability has been investigated in a framework of generalised SBP operators applicable to multiple dimensions and simplex elements. This last extension may be possible, but to the author's knowledge, there are no SBP operators on simplices in general fulfilling the conditions used there. Although these may exist, they will probably require more nodes per element and could therefore be less efficient.

Moreover, numerical tests have been performed using the flux differencing form and several different volume fluxes. There does not seem to be any clearly superior candidate outperforming the other ones in all cases. Whereas for smooth solutions some not entropy conservative volume fluxes performed better than their entropy conservative counterparts, this is different for the considered discontinuous solutions. Here, the entropy conservative volume fluxes yielded schemes that were more stable, i.e. that did not crash (due to negative density or pressure or other reasons). Nevertheless, coupling entropy conservative volume fluxes with dissipative surface fluxes is not sufficient for strong shocks. Thus, these results should be considered carefully, since no additional shock capturing mechanisms - which will be needed in practice - have been used.

Secondly, entropy conservative numerical fluxes can be used as surface fluxes in flux differencing form / discontinuous Galerkin / finite volume methods. There, they should be enhanced by additional dissipation operators. In section 6 , positivity preservation has been investigated. It has been proven that most of the entropy conservative fluxes preserve non-negativity of the density, if they are enhanced with local Lax-Friedrichs type dissipation operators.

Moreover, the (scalar, matrix, and hybrid) dissipation operators of Derigs et al. [6, 28] have been tested and compared with a simple local Lax-Friedrichs dissipation $-\frac{\lambda}{2} \llbracket u \rrbracket$ for the entropy conservative fluxes as well as with the classical LLF flux and the Suliciu relaxation solver of Bouchut [2]. In some problems, the hybrid and matrix dissipation operators yield similar results regarding stability and accuracy as the Suliciu solver, but they are less stable in general, as has been demonstrated in section 7.12. Therefore, the LLF dissipation $-\frac{\lambda}{2} \llbracket u \rrbracket$ seems to be advantageous compared to the scalar dissipation operator regarding stability, contrary to the results of [6] for the MHD equations, where specifically tuned dissipation operators were more stable than the LLF dissipation.

However, investigating performance of numerical fluxes, the costs have to be considered. Here, the implementation has not been optimised for every flux in detail, but the Suliciu relaxation solver is the second cheapest one after the LLF flux. The fluxes relying on an entropy conservative baseline flux are significantly more expansive. Thus, the Suliciu relaxation solver of Bouchut [2] seems to be the best one in this comparison.

There are many open problems. Firstly, the positivity of the pressure using the LLF dissipation has been observed in all test cases but no analytical proof has been conducted yet. Another possibility is the addition of dissipation for the variables $\varrho, \varrho v, \varrho s$ followed by a conversion to the usual conserved variables as described by Bouchut [2, Section 2.4.6].

Moreover, it has still to be investigated thoroughly in what regard the entropy conservative fluxes as ingredients in the flux differencing framework have advantages compared to the split forms tested by Gassner et al. [12]. Additionally, it is still unclear, whether there are some superior entropy conservative fluxes or cheaper ones.

Furthermore, the implications of (semidiscretely) entropy stable schemes have to be investigated. To the authors' knowledge, there are no general convergence results about high-order schemes for nonlinear systems of conservation laws in several space dimensions. Entropy stability, implying $L_{2}$ bounds if strict positivity of density and pressure are ensured, alone does not 
suffice to prove convergence, since such $L_{2}$ bounds cannot prevent oscillations can in general.

\section{Acknowledgements}

The author would like to thank the anonymous reviewers for their helpful comments.

\section{References}

[1] T. J. Barth. "Numerical methods for gasdynamic systems on unstructured meshes". In: An introduction to recent developments in theory and numerics for conservation laws. Berlin: Springer, 1999, pp. 195-285.

[2] F. Bouchut. Nonlinear stability of finite volume methods for hyperbolic conservation laws and well-balanced schemes for sources. New York: Springer Science \& Business Media, 2004.

[3] P. Chandrashekar. "Kinetic energy preserving and entropy stable finite volume schemes for compressible Euler and Navier-Stokes equations". In: Communications in Computational Physics 14.5 (2013), pp. 1252-1286.

[4] H. Chen. "Means Generated by an Integral". In: Mathematics Magazine 78.5 (2005), pp. 397-399.

[5] F. Coquel, E. Godlewski, B. Perthame, A. In, and P. Rascle. "Some new Godunov and relaxation methods for two-phase flow problems". In: Godunov methods. Ed. by E. F. Toro. New York: Springer Science \& Business Media, 2001, pp. 179-188.

[6] D. Derigs, A. R. Winters, G. J. Gassner, and S. Walch. "A novel averaging technique for discrete entropy-stable dissipation operators for ideal MHD". In: Journal of Computational Physics 330 (2017), pp. 624-632.

[7] D. Derigs, A. R. Winters, G. J. Gassner, and S. Walch. "A novel high-order, entropy stable, 3D AMR MHD solver with guaranteed positive pressure". In: Journal of Computational Physics 317 (2016), pp. 223-256.

[8] F. Ducros, F. Laporte, T. Souleres, V. Guinot, P. Moinat, and B. Caruelle. "High-order fluxes for conservative skew-symmetric-like schemes in structured meshes: application to compressible flows". In: Journal of Computational Physics 161.1 (2000), pp. 114-139.

[9] L. C. Evans. Partial Differential Equations. American Mathematical Society, 2010.

[10] T. C. Fisher and M. H. Carpenter. High-Order Entropy Stable Finite Difference Schemes for Nonlinear Conservation Laws: Finite Domains. Technical Report NASA/TM-2013217971. NASA Langley Research Center, Hampton VA 23681-2199, United States: NASA, Feb. 2013.

[11] T. C. Fisher and M. H. Carpenter. "High-order entropy stable finite difference schemes for nonlinear conservation laws: Finite domains". In: Journal of Computational Physics 252 (2013), pp. 518-557.

[12] G. J. Gassner, A. R. Winters, and D. A. Kopriva. "Split Form Nodal Discontinuous Galerkin Schemes with Summation-By-Parts Property for the Compressible Euler Equations". In: Journal of Computational Physics 327 (2016), pp. 39-66.

[13] S. Gottlieb and C.-W. Shu. "Total variation diminishing Runge-Kutta schemes". In: Mathematics of Computation 67.221 (1998), pp. 73-85.

[14] J. E. Hicken, D. C. D. R. Fernández, and D. W. Zingg. "Multidimensional SummationBy-Parts Operators: General Theory and Application to Simplex Elements". In: SIAM Journal on Scientific Computing 38.4 (2016), A1935-A1958.

[15] F. Ismail and P. L. Roe. "Affordable, entropy-consistent Euler flux functions II: Entropy production at shocks". In: Journal of Computational Physics 228.15 (2009), pp. 54105436 . 
[16] A. Jameson. "Formulation of kinetic energy preserving conservative schemes for gas dynamics and direct numerical simulation of one-dimensional viscous compressible flow in a shock tube using entropy and kinetic energy preserving schemes". In: Journal of Scientific Computing 34.2 (2008), pp. 188-208.

[17] C. A. Kennedy and A. Gruber. "Reduced aliasing formulations of the convective terms within the Navier-Stokes equations for a compressible fluid". In: Journal of Computational Physics 227.3 (2008), pp. 1676-1700.

[18] Y. Morinishi. "Skew-symmetric form of convective terms and fully conservative finite difference schemes for variable density low-Mach number flows". In: Journal of Computational Physics 229.2 (2010), pp. 276-300.

[19] S. Pirozzoli. "Numerical methods for high-speed flows". In: Annual review of fluid mechanics 43 (2011), pp. 163-194.

[20] H. Ranocha. "SBP operators for CPR methods". MA thesis. TU Braunschweig, 2016.

[21] H. Ranocha. "Shallow water equations: Split-form, entropy stable, well-balanced, and positivity preserving numerical methods". In: GEM - International Journal on Geomathematics 8.1 (Apr. 2017), pp. 85-133. DOI: 10.1007/s13137-016-0089-9. arXiv: 1609.08029 [math.NA].

[22] P. L. Roe. Affordable, entropy-consistent Euler flux functions. Talk presented at the Eleventh International Conference on Hyperbolic Problems: Theory, Numerics, Applications. Lyon, 2006. URL: http://www2 . cscamm . umd . edu/people/faculty/tadmor / references/files/Roe_Affordable_entropy_Hyp2006.pdf.

[23] C.-W. Shu. Essentially Non-Oscillatory and Weighted Essentially Non-Oscillatory Schemes for Hyperbolic Conservation Laws. Final Report NASA/CR-97-206253. Institute for Computer Applications in Science and Engineering, NASA Langley Research Center, Hampton VA United States: NASA, Nov. 1997.

[24] G. A. Sod. "A survey of several finite difference methods for systems of nonlinear hyperbolic conservation laws". In: Journal of Computational Physics 27.1 (1978), pp. 1-31.

[25] E. Tadmor. "Entropy stability theory for difference approximations of nonlinear conservation laws and related time-dependent problems". In: Acta Numerica 12 (2003), pp. 451512.

[26] E. Tadmor. "The numerical viscosity of entropy stable schemes for systems of conservation laws. I". In: Mathematics of Computation 49.179 (1987), pp. 91-103.

[27] E. F. Toro. Riemann solvers and numerical methods for fluid dynamics: A practical introduction. Berlin Heidelberg: Springer, 2009.

[28] A. R. Winters, D. Derigs, G. J. Gassner, and S. Walch. "A Uniquely Defined Entropy Stable Matrix Dissipation Operator for High Mach Number Ideal MHD and Compressible Euler Simulations". In: Journal of Computational Physics (2016).

[29] P. Woodward and P. Colella. "The numerical simulation of two-dimensional fluid flow with strong shocks". In: Journal of Computational Physics 54.1 (1984), pp. 115-173.

[30] X. Zhang and C.-W. Shu. "Maximum-principle-satisfying and positivity-preserving highorder schemes for conservation laws: survey and new developments". In: Proceedings of the Royal Society of London A: Mathematical, Physical and Engineering Sciences 467.2134 (2011), pp. 2752-2776.

[31] X. Zhang and C.-W. Shu. "On positivity-preserving high order discontinuous Galerkin schemes for compressible Euler equations on rectangular meshes". In: Journal of Computational Physics 229.23 (2010), pp. 8918-8934. 